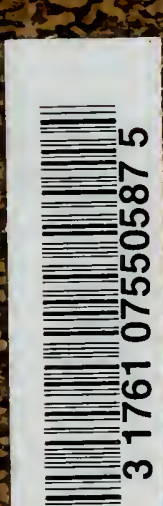

(2)

a

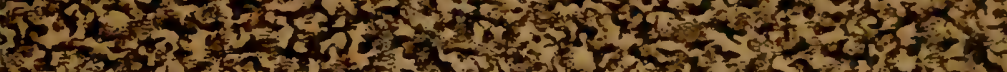

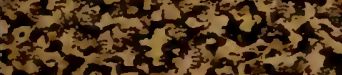

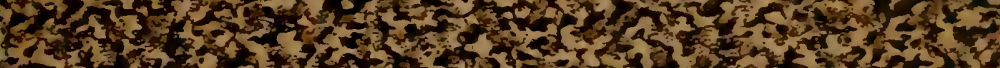

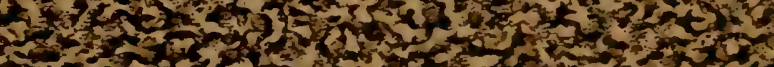

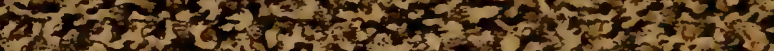

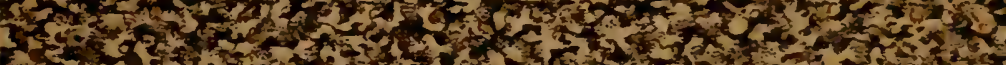

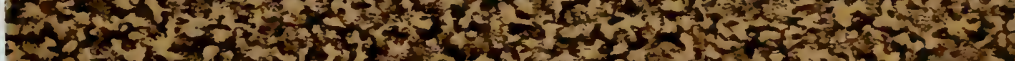

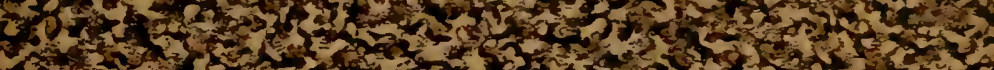

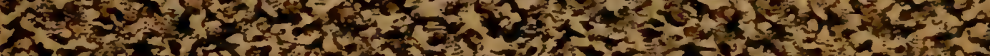

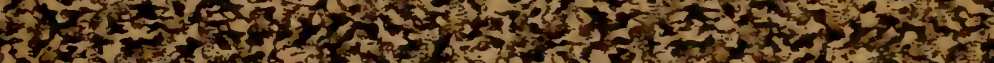

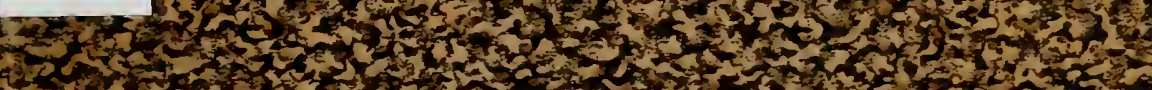
(2)

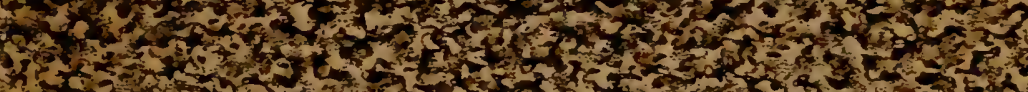

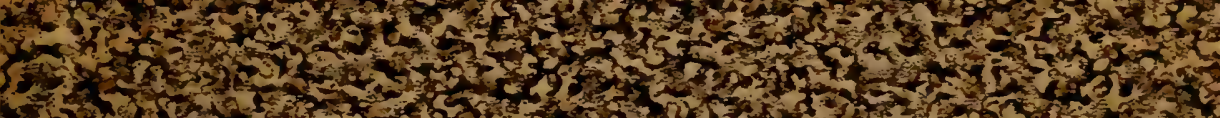

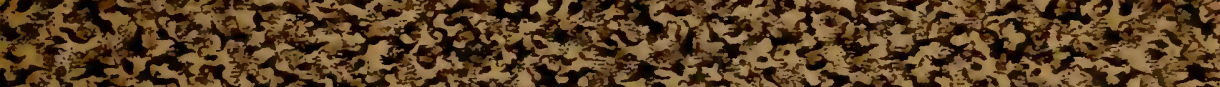

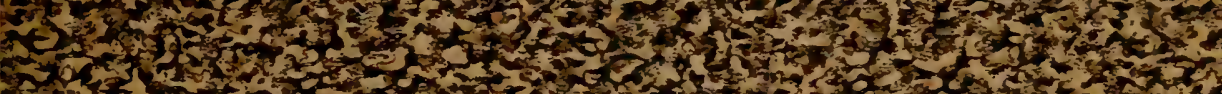

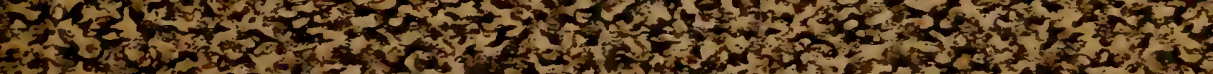

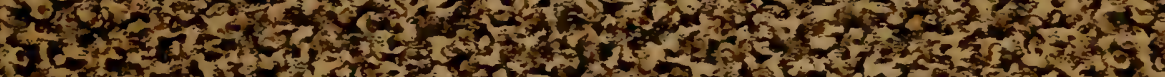

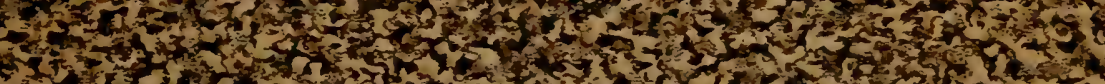

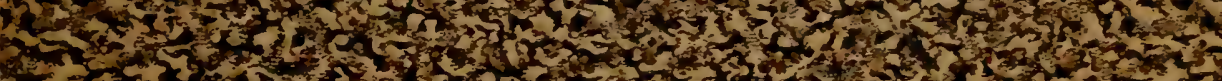

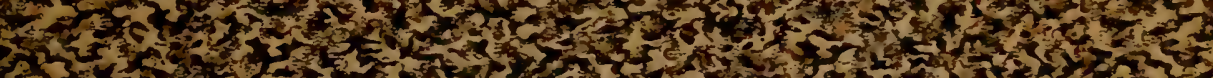

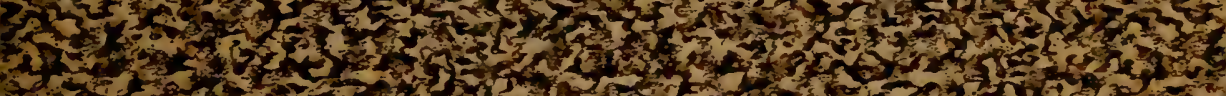

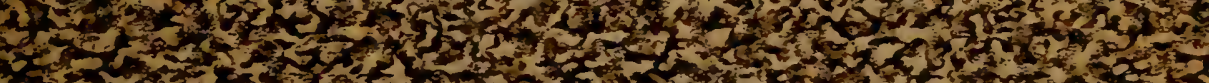

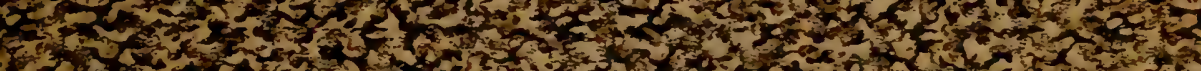

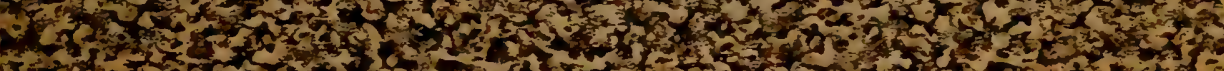

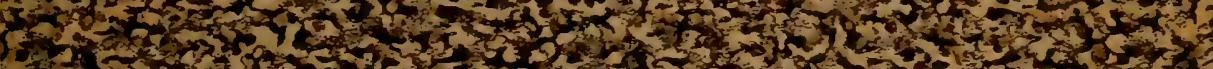

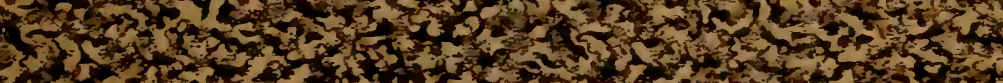

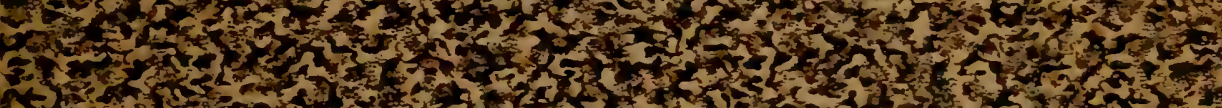

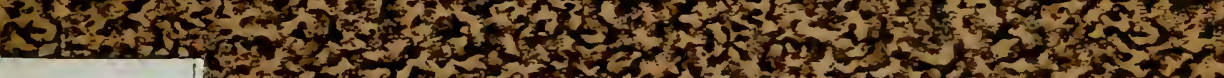

SD

393

B4

ग्रा

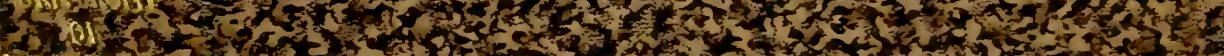

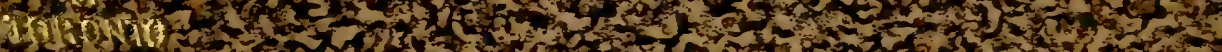
for 



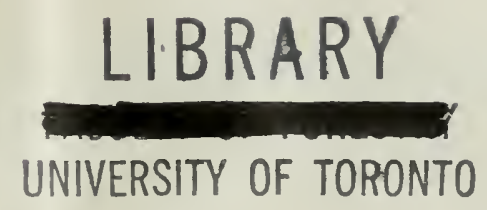





\section{Lleber don Einflur}

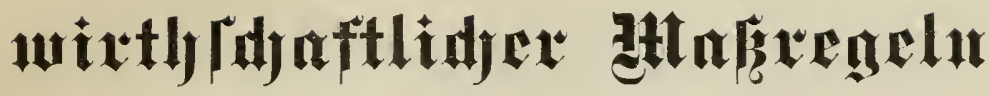 \\ anf}

อెּwachsverhältniffe nuto 2Rentabilität

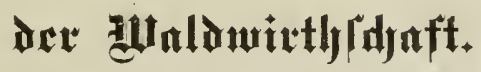

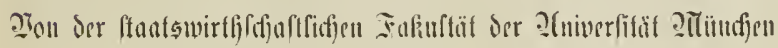

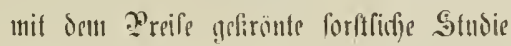

ron

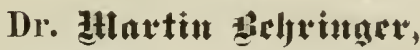

Rönig!. bavr. Foritnmtहaififtent.
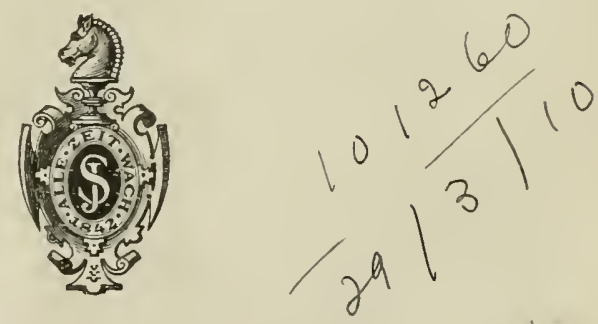

อ̧extir.

Derlag vou Julius Spriuger. 


$$
\begin{aligned}
& \text { SD } \\
& 393 \\
& 34
\end{aligned}
$$




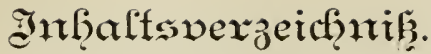

Seite

Ëinleitung

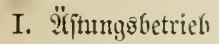

II. Yeinigungshetrieb

III. Durdforjtmngebetrieb . . . . . . . . . . . . . . . . . 11

1) 3uwathisgantg in Bejug ant:

a) Beptandesitürfe . . . . . . . . . . . . . 12

b) Şäge und Etammjorm . . . . . . . . . . . . 25

c) Bejammtmaīe . . . . . . . . . . . . . 30

2) Mientabilität im $\mathfrak{I}$ Ifgemeinen . . . . . . . . . . . . . 40

a) im autijeţentom betriebe . . . . . . . . . . . 41

b) im Paadgaltbetriebe. . . . . . . . . . . . 48

IV. Rifutungafetricb. . . . . . . . . . . . . . 54

1) 3mwadgrgang im $\mathfrak{U}$ (fgemeinet . . . . . . . . . . . . 55

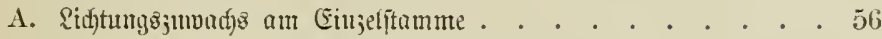

B. = an ganjen Bejtänoen. . . . . . 63

2) Mentabilitït im Iffgemeinter . . . . . . . . . . . i1

a) im aนşetzenten Betriełe . . . . . . . . . $\%$

b) im शadghaltfetriebe. . . . . . . . . . . it 



\section{Einleituthg.}

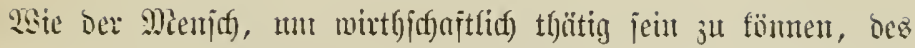

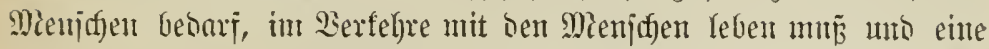

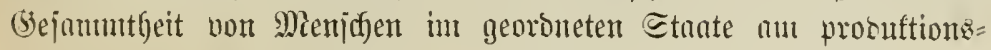

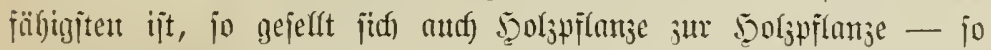

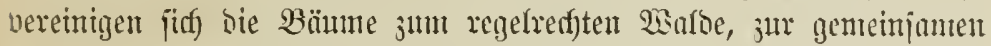
mo barm un jo gedeiffideren Firobuftion. Şier wie bort ijt ser Sampi tun bas Dajein burd) Das 3njannuenleven uno ben greiden

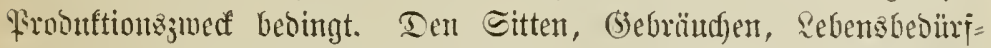

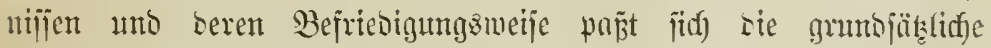

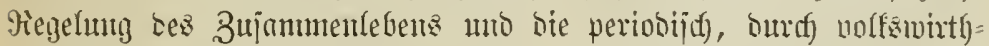

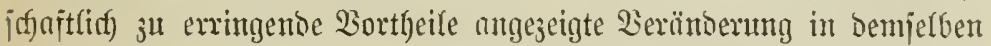
an - bie Eigenjanjten ber jun gemetujidajtlidgen Bejtonos leben

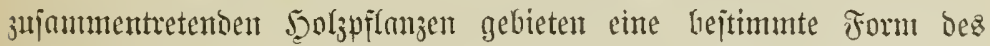
3ujammentebens uns verlangen, jofern ber nationale Wablifant ge=

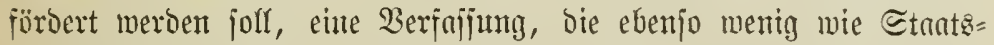

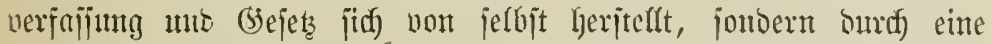
autoritative Gewalt hergejtell uno bejtündig regulirt werben mús. গ⿰氵

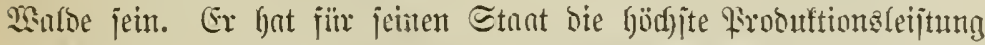

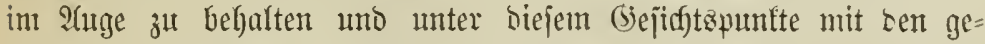
gehenent Berbältnifien redfneno Conber= unt Gejannt = interejie jeiner lluterthanen genenjeitig abzumägen, in Einflang ju bringen

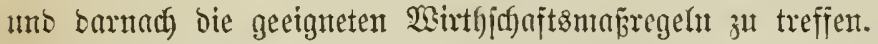

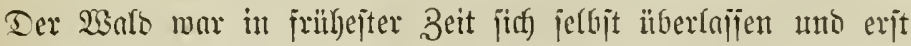

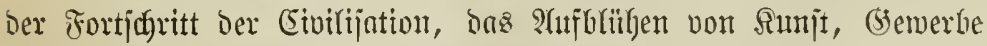
uno Indurtrie zmängte benjelben in bejtinmte Formen, benen bie

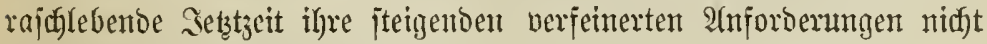
rajch gentrg abgerwimmen fann.

Die Ummandung in ber $\mathfrak{W}$ aldowirthifgaft gegen früfer volfjog sebringer. 


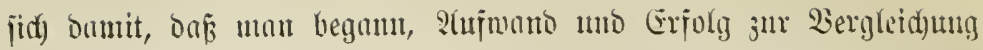

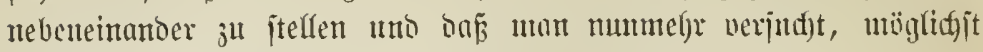

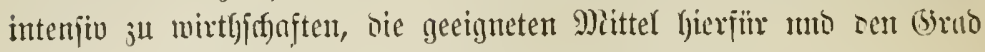

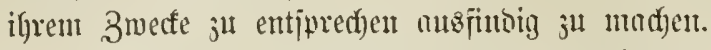

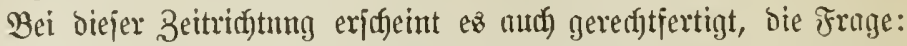

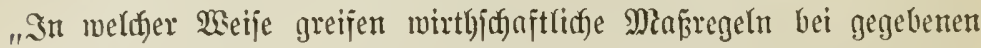
natïrlidifen Stunbortsaftoren in ben Bumadfiganty ber Bäume unto

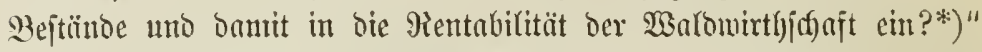
oufzumerfen uno einer nälyeren Eriorterung ju untergieljen. Dieje

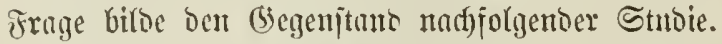

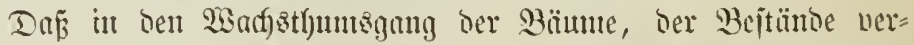
änberno eingegriffen werben faun unb unter llnijtïnten eingegrifien

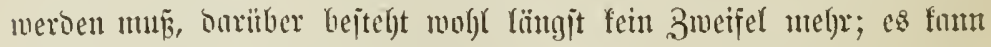

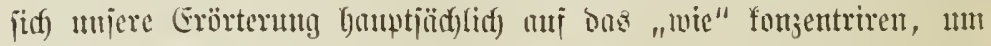

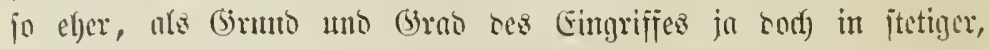

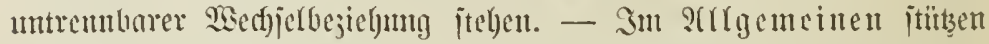

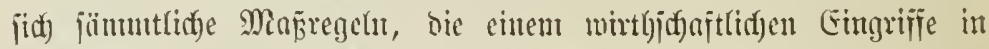

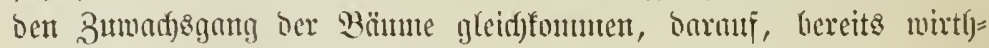

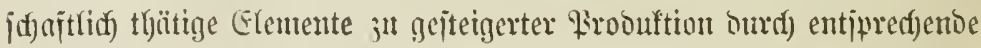

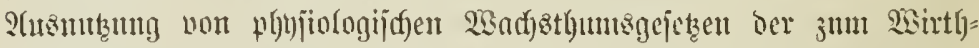

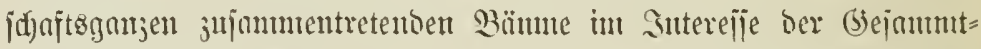

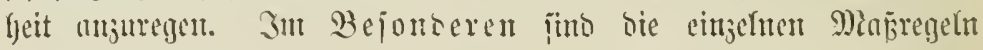

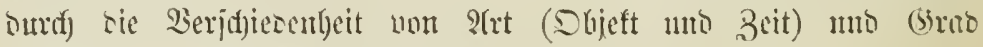
(Stanoroun) bes (Eingrifijes d)arnatterifirt.

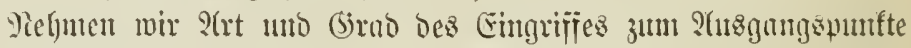

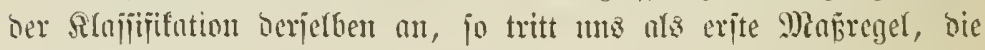
jidf) แteijt un anj amsertejene, bontinirentoe Etïnnme mo zmar leoig=

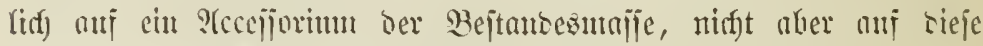
jelbjt, jidf eritrecft - bie sc ejtung entgegen.

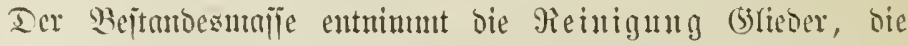
won P(njant an tem defintiven Bejtanbesbitoe nidjt jugebad)t jein

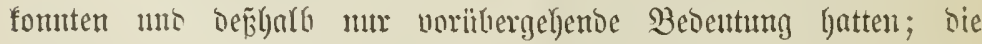
Durdforitung ragegen entient je nad) bem (stabe bie nntljütigen,

*) Wortlaut ber non ber ftaat8ıirthiđaftl. Fafultät Mändjen pro $1889 ; 90$ gefterten Breisaufgabe. 


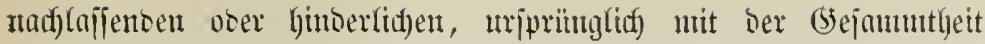

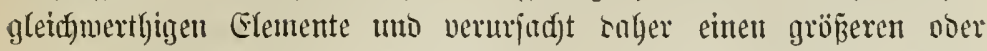

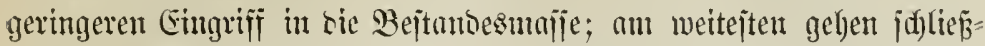

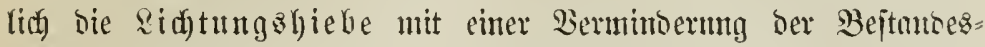
glieber zll Gumiten meniger bevorjugter Snbiviouent uno mit ciner onnernten Luterbredfung bes Bejtantesiffinfes.

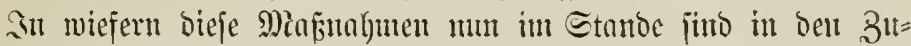

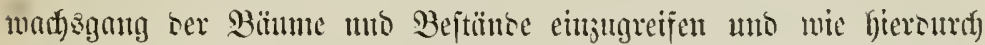

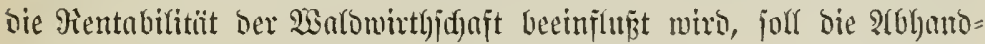
fung Der einjeltuen Maßjregefn jelbjt zeigen.

\section{Alfutugsbetxieb.}

Die Forjtwirtbjiffart unteriffeibet nad) Dent Materiale, meldyes

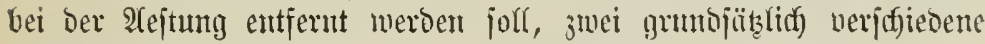

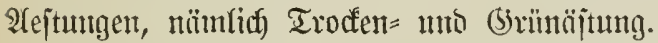

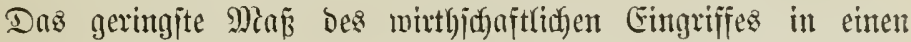

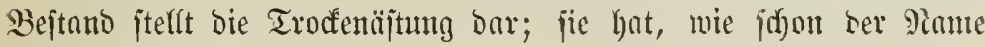

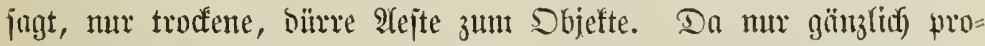

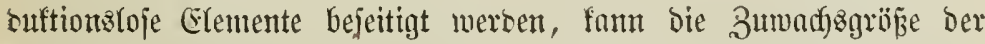
betroffenen Bäume nidgt beeinflupt werben uno inzbejonbere feine

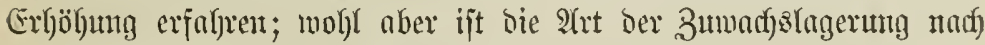

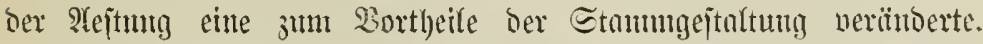

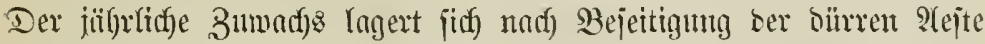

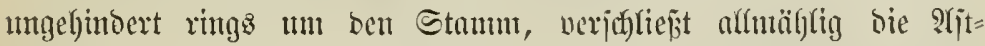
munben un ver(eilyt bem Stamme, da sas Cimmachjen ber tobten

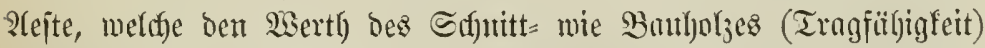
erfheblidy oriuffen, vermiesen ijt, cinte ajtreine, watjige bsejtalt unto

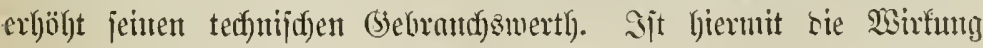

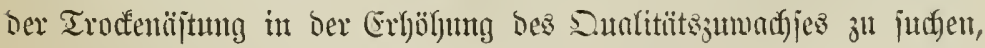

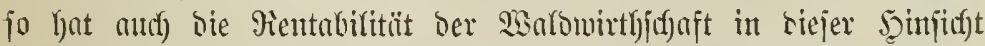
nübjit ben in Betrad)t fommenoen 2(ejtungafojten bie betreffertoen Suafitütosbifferenjen zu head)ten. 
2(

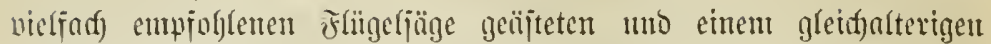
midft geäpteten Etanme in J̄anbarfeitzalter (won 80 Sabren) nuf 7 Maf. uno berectnet bent (seminn nuf $62 \%$ von Bruttoertrage.

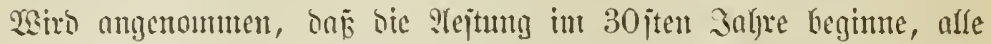

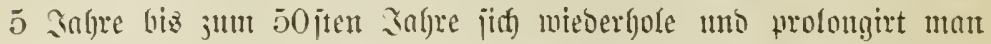

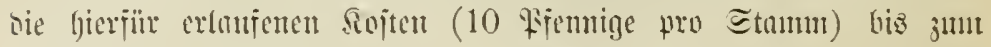

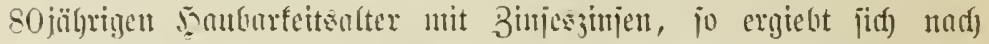

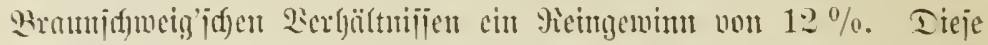

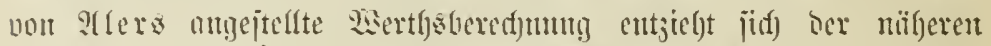

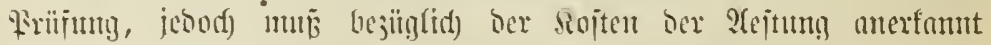

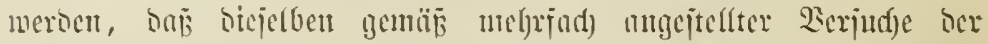

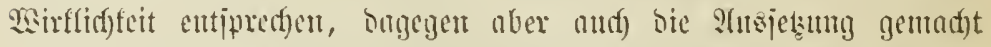

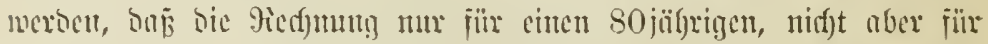

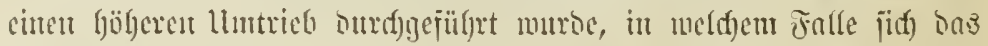

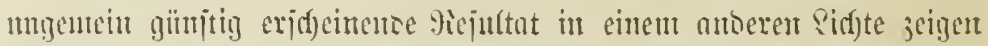

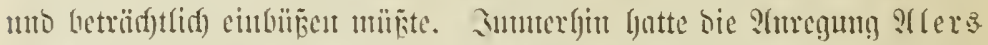

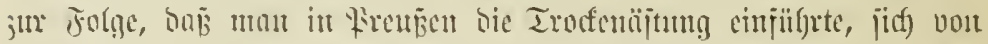

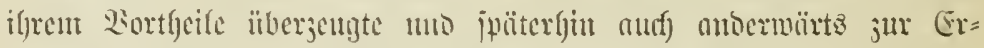

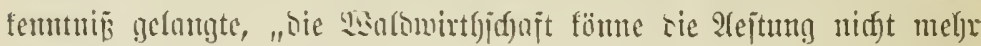
enthel)ien" $"$ *\%; $)$.

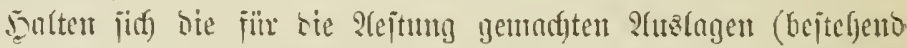

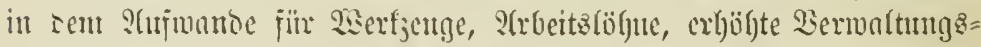
fojtert) mo ber crutidfte Erjoly (höberer Erlöz fïr baz geäjtete

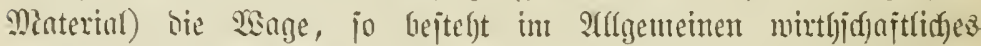

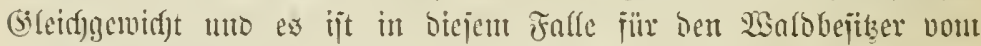

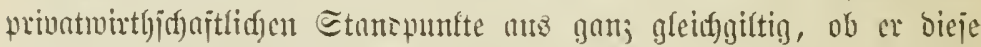

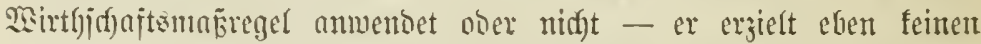

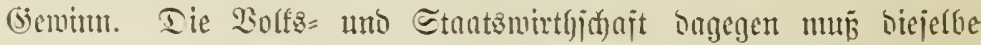
menen ber Erjeugung einer gröjeren E.unntitït glattjogatigen, gut

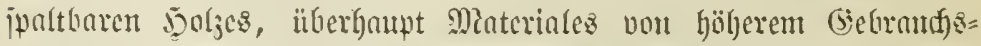
mertf)e uno megen ber (jemïgrung bon irbeitsueroienjt als rentabel begriǟen.

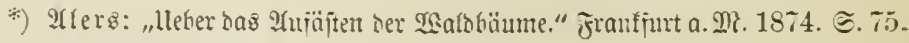

***) Eäd)ijiger Foritherein $15 \%$. 
Die Pentabilität Der Rejtung jteigt mit affen jentent Miomenten,

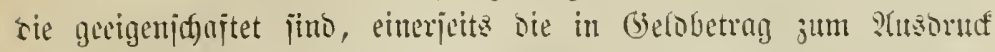

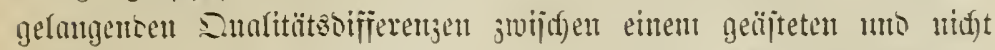

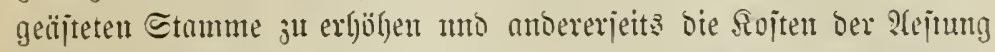

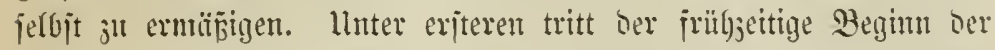

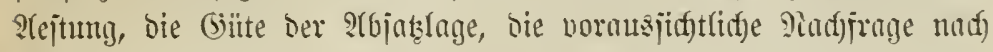

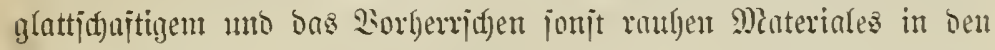
Borbergruno; bei ben Ketzeteren sagegen ijt bie Bermentung gejuidfter,

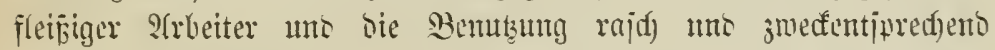

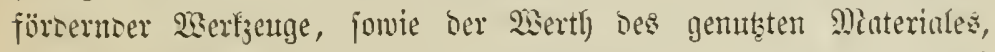
foierne ein joldjer vorganden ijt, aușjulaggebento. Ferner mun nod)

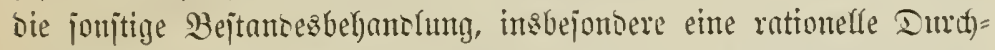
forjtung nor ber Rejtung, Durdy weldye bie Trofentïjung auf

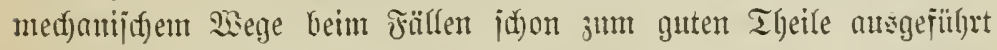

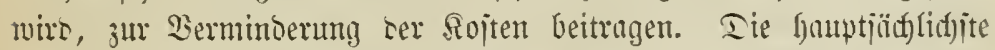

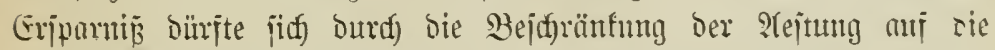

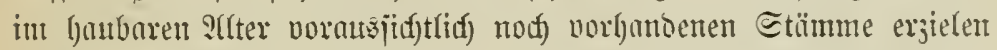

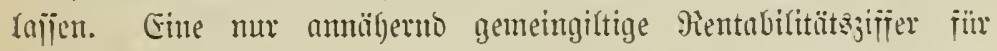

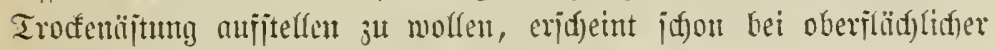
Erwüigung uno Bergleifung gennunter Faftoren als ein Ding oer

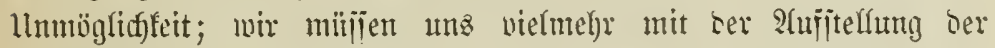

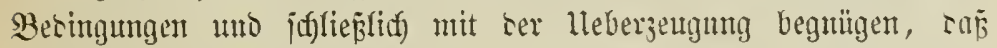

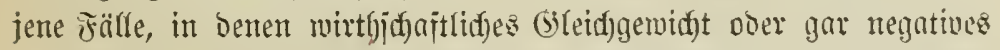

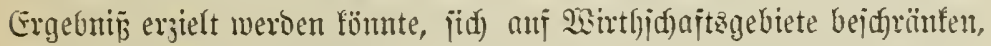
meld)e ïber(fartpt nod feinem intenjioen Betriebe mo bager andf ber 2(ejtung nidft zugüngliđa jirts.

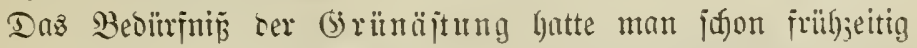

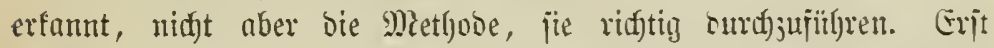
Sicomte be Courval, De Car beanjuruden bas Berbiemit, gegen bie bis in sieje Beit vie(fad) gebräudfitid)e 2erejtitmmelungsmanter

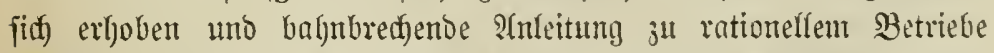

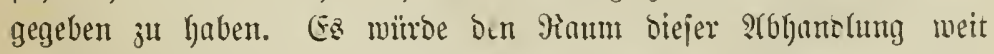

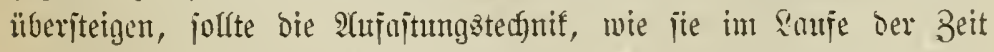

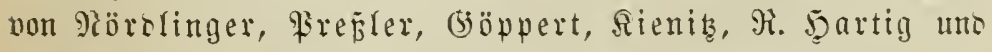

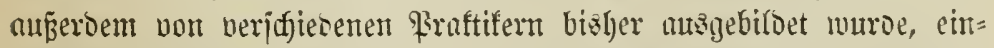
gelyeno beiprodjen werten. Die vertangte gientubilität ber Alejtung 
jekct eben Den rationellen Betrieb vorans unto erfordert, buj bie ge=

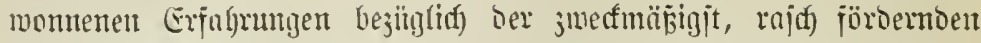

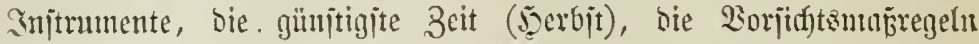

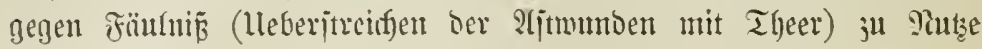
gemad)t merben und bie 2(ejtung jelbit bie juläjijge Grenje in Ironen= löbe mo acjtitürte nidgt überjogreite.

Die Entnnfyme non grïnen aejten legt bie Siermutfuntg nube,

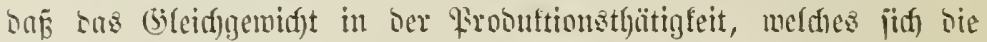
Datm bis jum Beitumfte ber 2lejtum im Zujammenmirfen wou

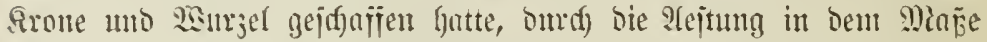
geptibt werbe, als bie Biloungsitoife erjengenten Blattorgane ver: mintert merten. Es ijt jebod) fincsimegz immer afs anbebingte Fulge der 2lejtung eine Bmmad)zulungme ju fonjtativen; biejelbe fann mir eintreten un miro jidfer cintreten bei gejteigertem Diape sev

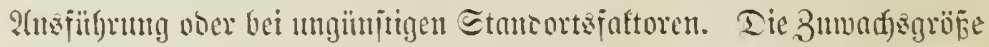

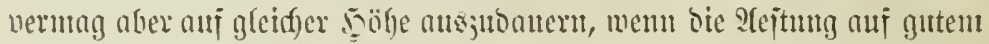

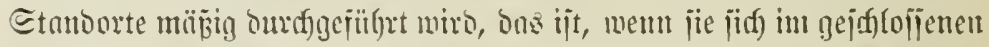
Beitance aui Bmeige mit jajt unthütigen Blattorganen bejh)ränft.

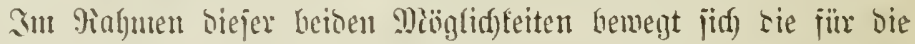

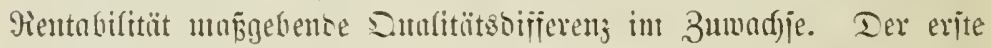
Fall ijt in jeiner fmonomentalen saifung gleidjbebentento mit ber

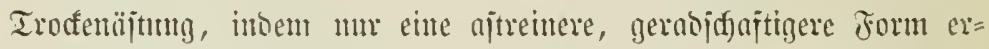

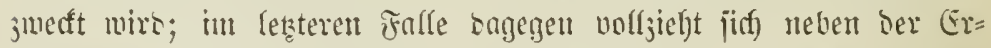

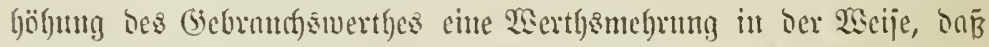

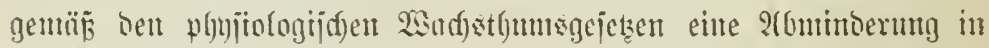

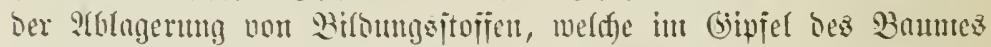
begimnt uno mad) unten ji(f) jurtjetst, nuj Sojten rer unteren Stanmu=

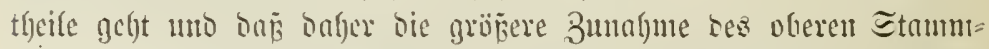

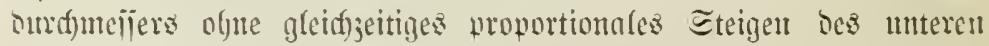

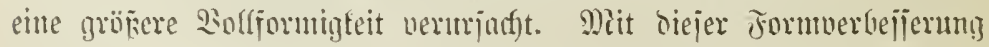

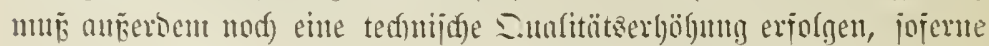

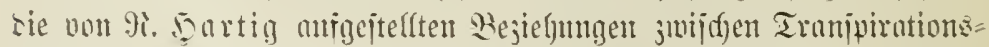

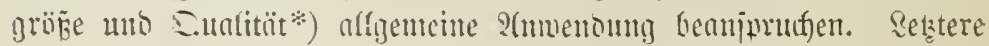

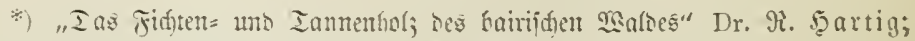
Eentraftalt $ं$. D. gejammte Jorjtuejen 1888. ₹. 363. 


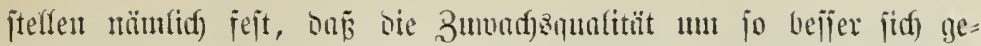
jtaltet, je mentger bie 3mmaffsutenge im Berbältnilje jur Tramjpi= rationsgröß̈e jütt.

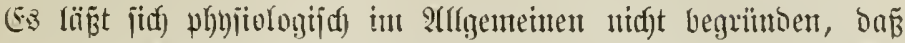

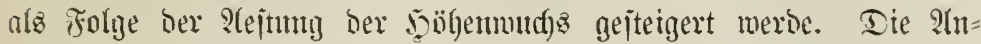
fidfen uno (Erfahrungen geben in biejer Şinjuft anzeinanoer.*)

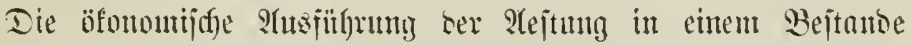

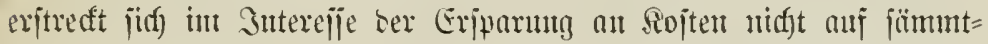

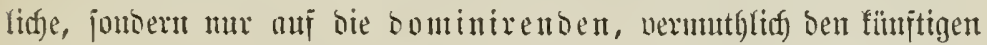

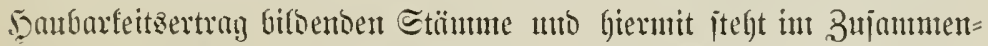

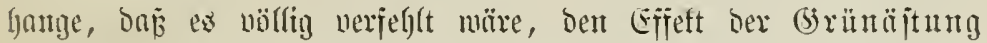

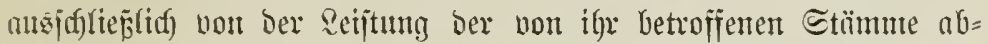

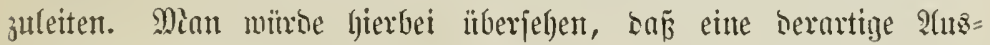

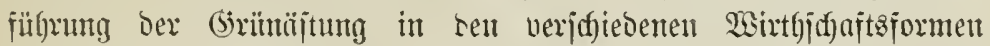

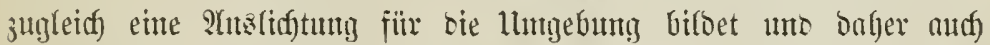

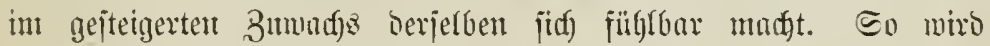

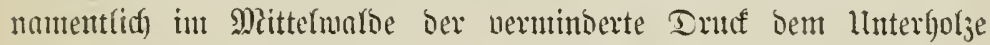

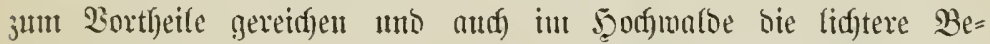

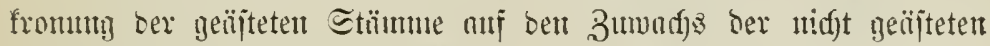
Jinct)barptüume gïutitig cinmirfent.

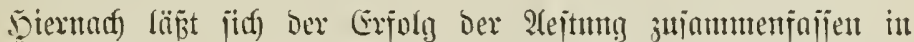

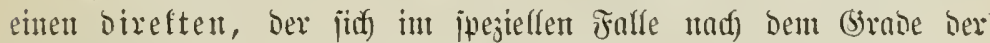

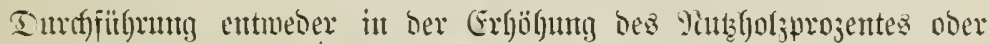

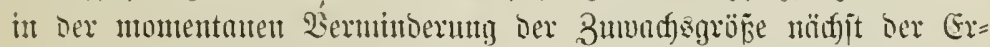

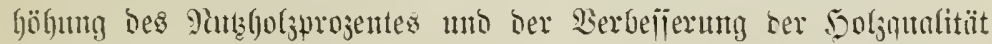
ausjprift; unt in einen inbireften, ter in bem entiprechento ver: meforten Bumadjs ber llngebung ju judfen ijt.

Sie Situratm enthält über bie 2lejtungserjolge fïr unjer Thema nun jegr menig. Sas hieritber Seröffentfidfte bejielgt jidj loaupt=

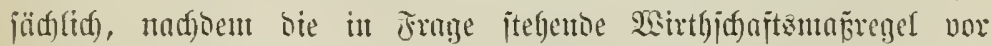
menigen Iejentien erjt grïnthif) nentilirt uno in neuejter Beit audf non ber firaxis exuthajt in bie hano genounten ijt, barauf, bie

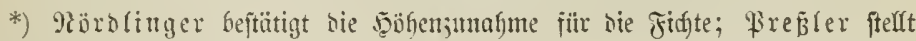

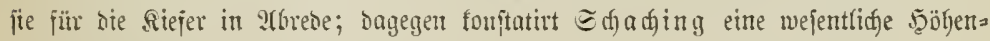

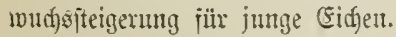




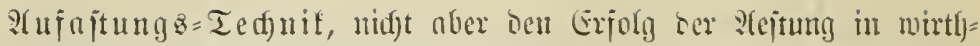

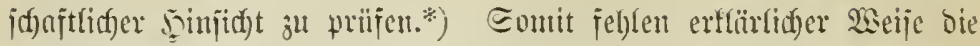

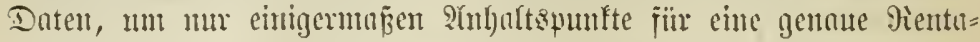

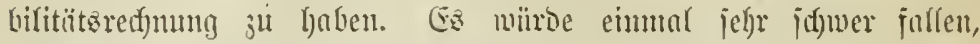

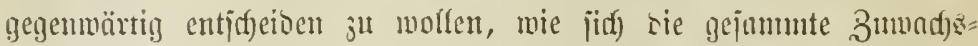

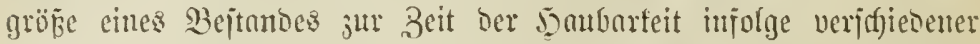

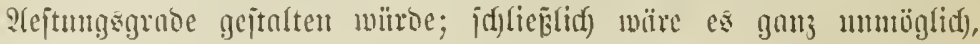

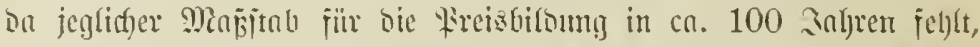

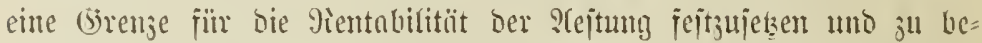
ftimmen, weld) bö̈gerer Freis oer Eintheit gen̈jteter Minjīe jufoumen

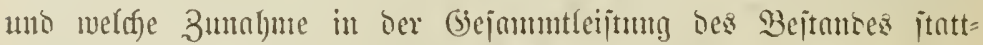
Finsen milife, onmit jiberfoupt bie sojten geoceft mïrsen - ober

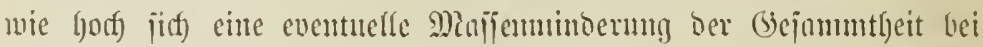

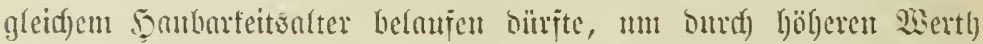

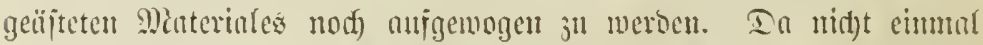

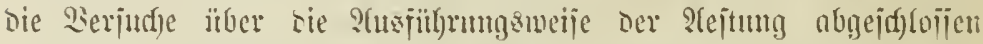
jimo uno nod) meniger itber ben Erifolg Biejultate norfiegen, fo ent=

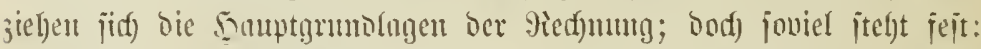

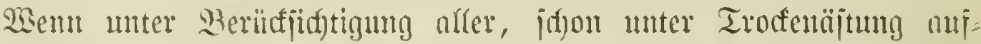

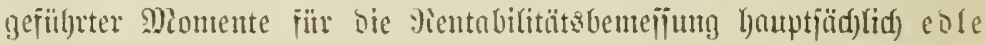

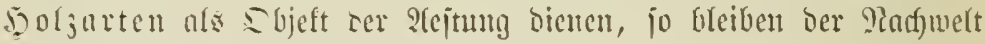
Biloer, wie jie tiej herabbenitete morjde alte Eidjen bieten, erjpart

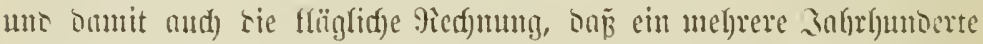

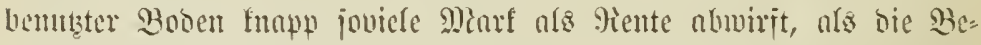

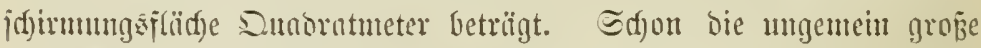

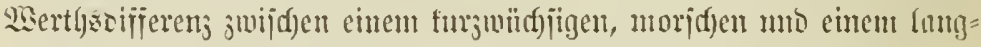

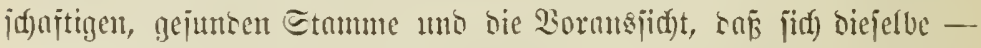

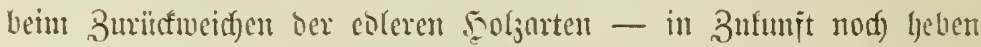

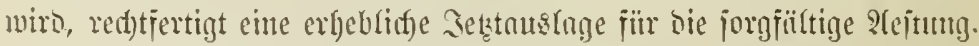

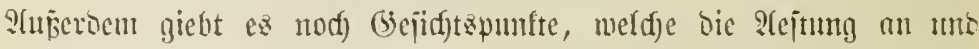
fïr jid) rentubel erjajeinen Injןen; fie geljören zwar, jtreng genoumen,

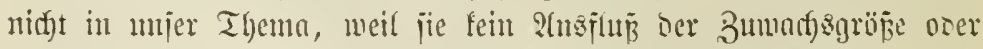
=(biute jino, jontern melgr negntiven Ermügungen entipringen; wir glauben jebod) biejelben in ?anbetrad)t ber lyoben Besentung, wethe

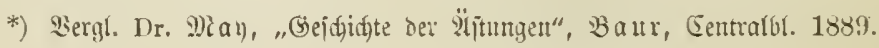




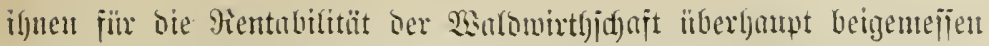

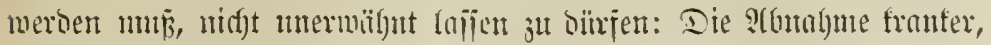

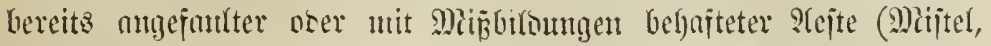
Sexcubejen zc.) nermeiset ein llebroreijen ber fruttgeit in sen

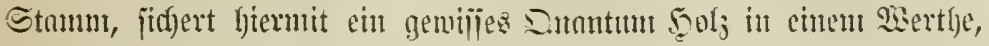

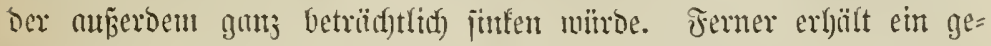

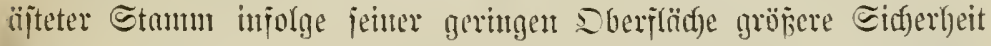

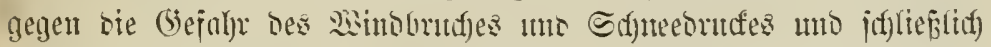

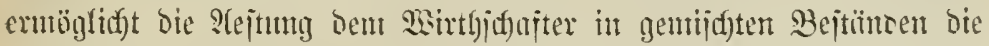

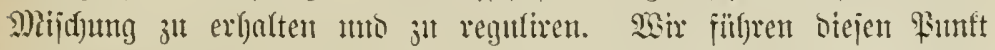

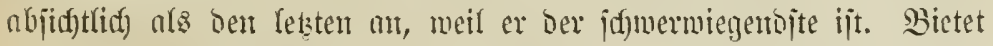

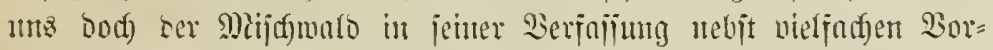

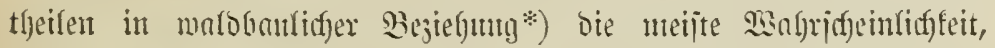

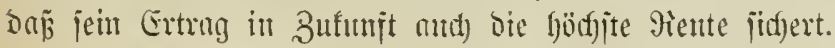

\section{Zienrigutugshetrieb.}

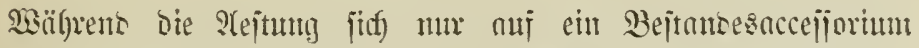
eritrecft, entrinmt bie Reinigung sem Bejtante woribergehento jut=

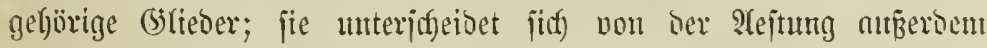

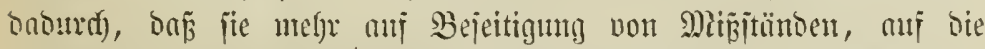
Seeritel(ung oer northeiflyojteiten Bejtonoesgejtaltung uno bantit in= bireft audf) nui eine Bumad)siteigerung abjielt. Wir jitfrent biejelbe

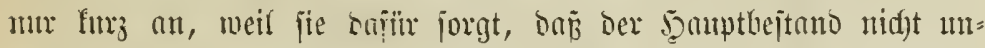

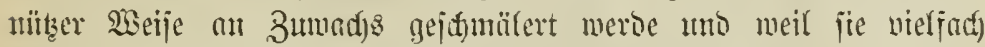
für bie Rentabilität von hervorragender sistftigfeit ijt.

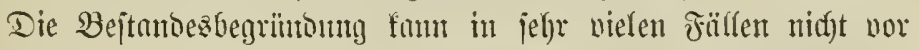

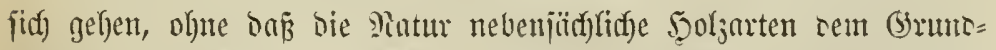

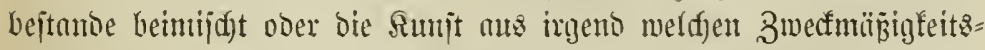

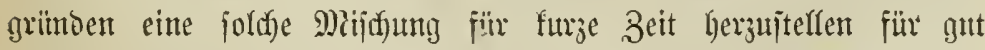

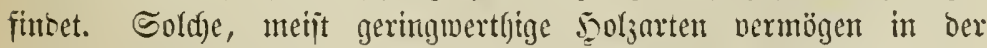

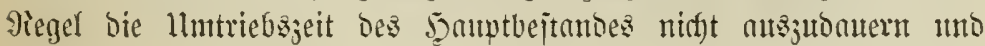

*) Dr. (s) ayer, der gemijijte WSato. 


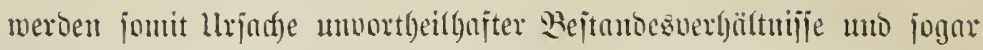
theilmeijer Firoduftionslojigfeit bes Bonens, eriüllen nber anbrerjeits

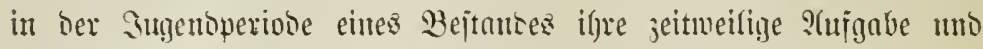

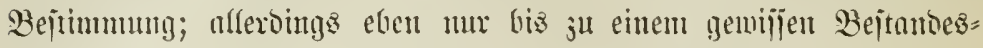

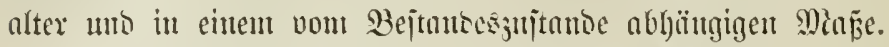

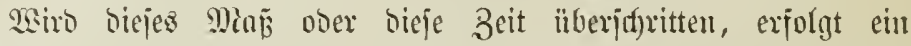

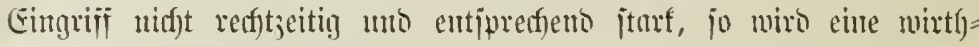
idfajtlidfe Siunce begangen, bie jidf jmax erjt ipät, aber un jo jufwerer rïrfyt.

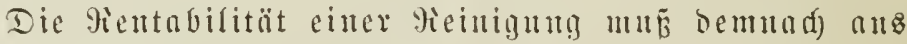

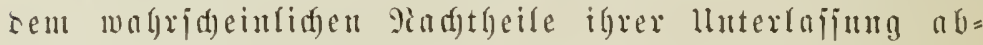
jtragirt weroen.

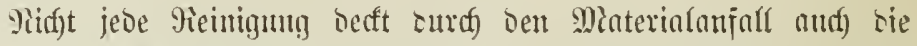

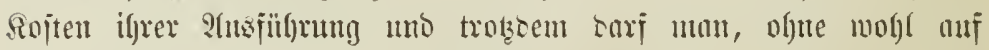

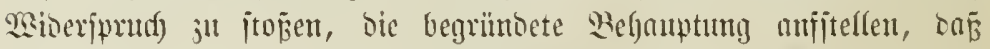

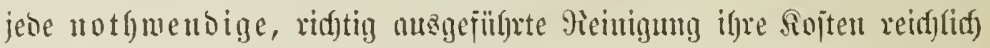

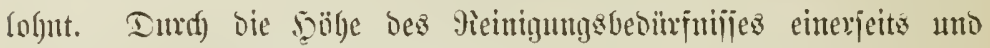

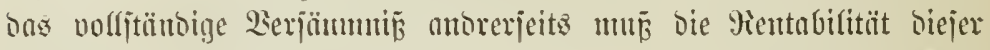

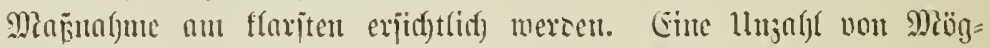
(idffeiten ijt jmijgen biejen beiben Extremen gelegen, je nadf beun

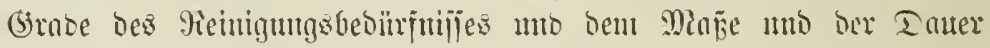

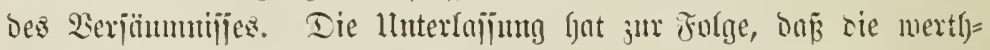

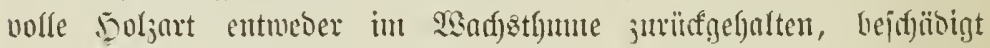
(burd) qieitjden :c.) voer uollitänbig ïberwadjen miro unt an beren

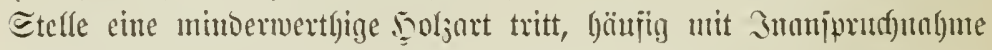
eines Bobemrames, ser mit Dem barauf probujirten Minteriafe in

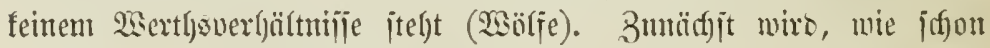

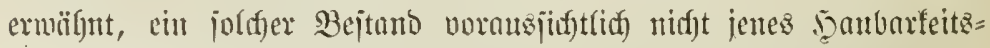
niter erreicfen, bas igm ofyne sie Beintengung ber Meinigungsitüume jutgefommen märe, uno samit liefert ber Bosen andf) nidjt jente volfe

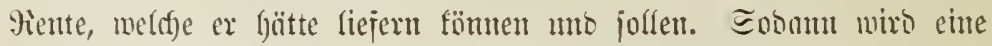

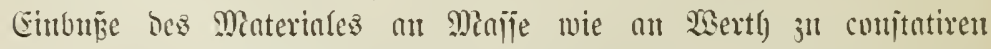

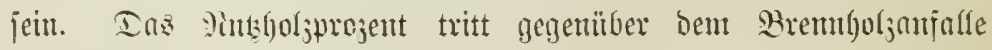

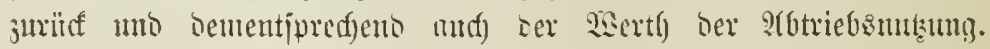

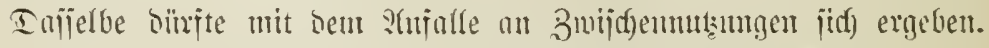

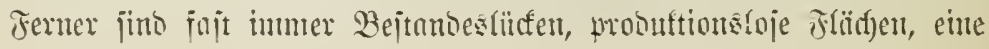




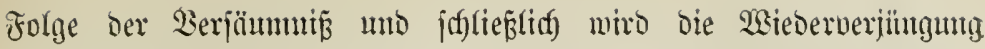
eines nifft geeignet gereinigten $B$ ejtandes, wenn man ber jtandortz=

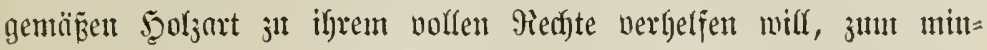

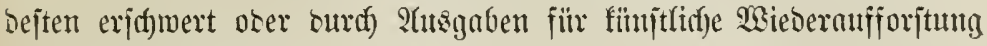
mejentfid) nertleutert.

Wätroe ez gefingen, an einem exaften Beijpiele bie Ertrïgnifife eines nad) den Miegeln bes 2 sarbonues gereinigten uno eines ver= nadfläjiinten Bejtanbes in (5elo anjujetzen unto and) nod) bie jonjitigen

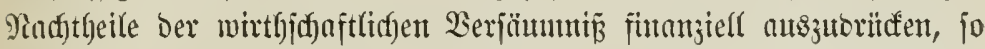

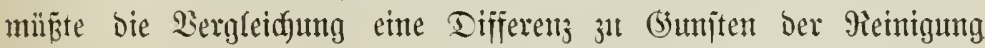

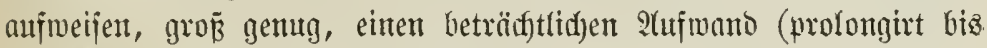

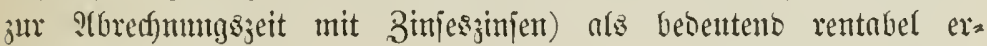
jofeinen ju lajien.

\section{Duxdffoxlfungs}

Die Bergleidfunt eines in bifftgejffloijenten Bejtanbe ermadfienten

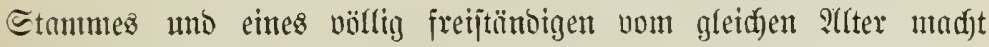

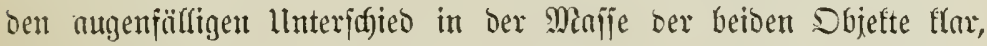
fülfyt aber audf) ju ber lteberzeugung, ba

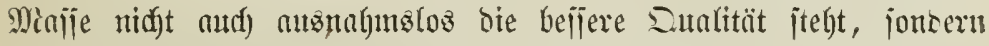

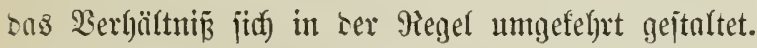

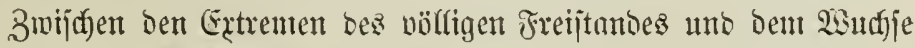

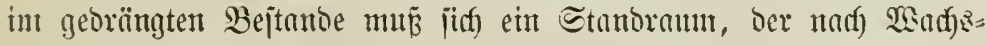

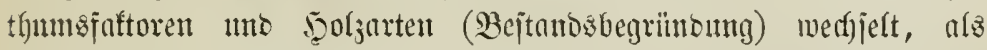
Mittel finoen, bei weldfent bie Majife ein Mancimum erreidft, ofgne on bie Dunfität Derjelben eine Cintonß̌e erlitte.

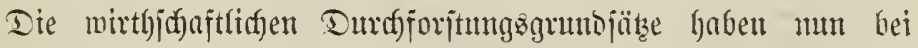

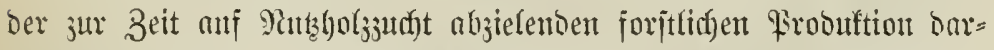

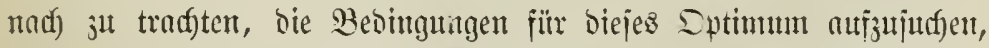

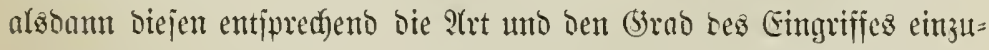
ridften und ben verffjiebenen Rebenzperioden eimes Bejtandes anjupaijen.

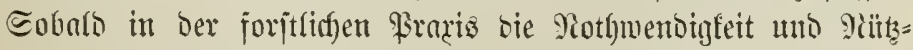
fidfeeit ber Durdforjtung inferbaupt eingejegent murte, madfte fidf 


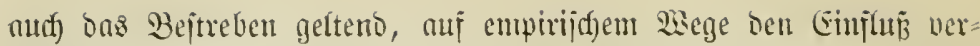
jajiebener Iurdjorjungsెmeije ju ermitteln mo biejen Bejtreben nerbanfen mir bas Entitehen viefant ausemander gehenter Thenten.

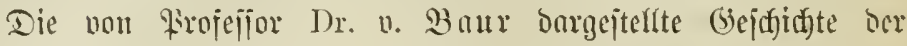

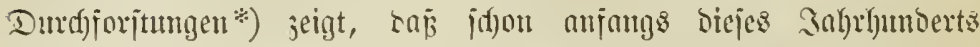

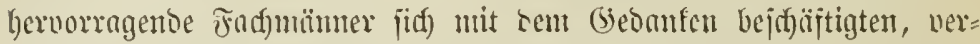

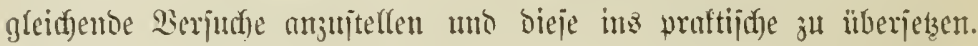

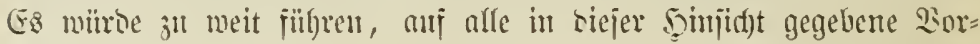

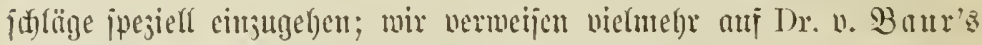

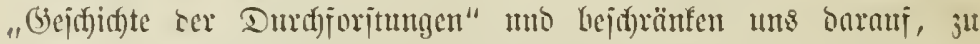

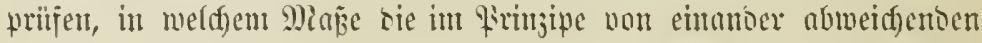

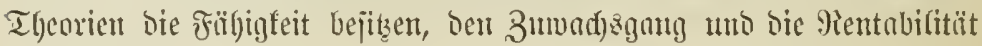
з̆ beeinjūjen.

Edjon (3). S. Jartig mo jein Beitgenoje Entta maren bie

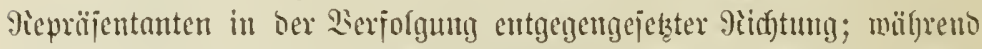

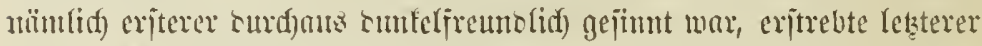

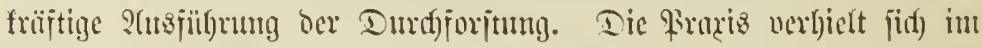

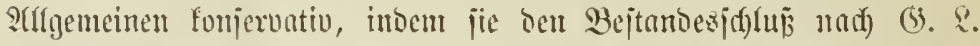
Jartig's (5rmbojïzen ju mafren trad)tete. Vicuertings bringen Dr. Borggreve mo 2 anguer, jeoer in eigentiger 2seije, ourd)

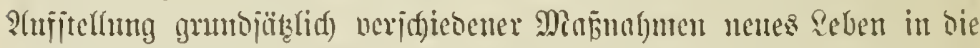
Turdjorjtumgsfruge. Seine ber beioen Theorien hat bis jetzt joldt)

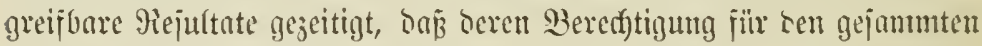

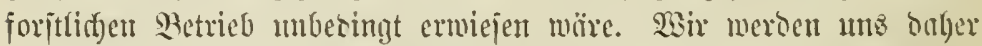
mit benjelben mu nom Etanopunte ber Theorte an bejä̈jtigen, in ser Sauptjatbe aber bie llnterjufungen in Sinne ber risber

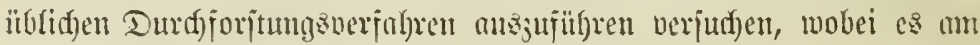

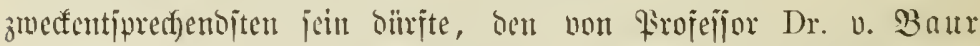

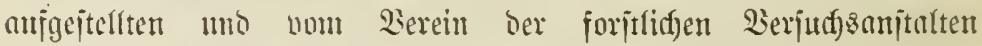

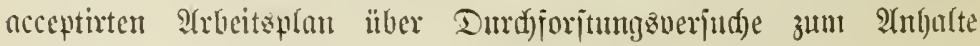
zit neluten.

Die Fragejtelfung des vorwinrigen Thentas gliebert jelbjt jufjon

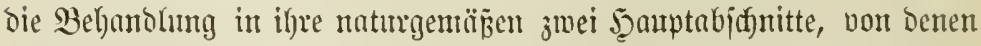
ber erjte bie art bes 3umadgangez, ber zmeite bie hiernon ab=

*) Banggofer, foritt. Berpudgivejen Bo. II. G. 216 fi. 


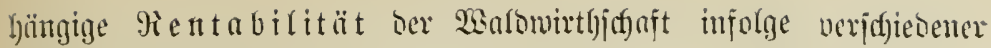
Dunfojorjtungsimeije jum (Segenjtante gat.

\section{1. èmundjgnnug.}

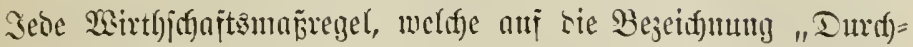

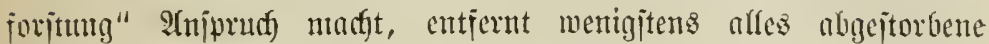
Miaterial ats bem Bejtande no wolfjielyt Giermit nur cinte Ernte, melde bas Bejtandeşleben burd) jeinen Entmicfelnngsong jelbjt ge=

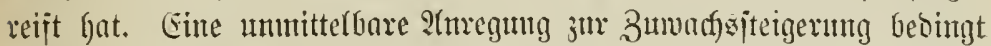

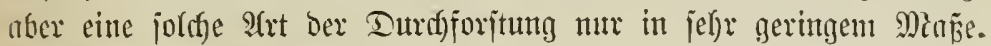
Eryt ber Eingriff in Den Rronemram der lebensfäbigen Stünme giebt

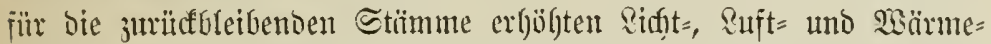

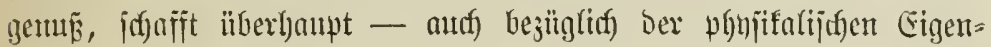

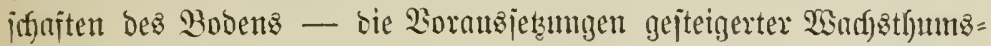
reijtung.

Sollen gegenuiärtige lluterjudumgen einen bentidgen Einflicf in

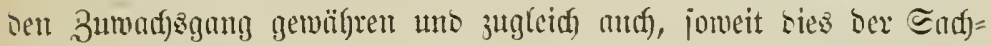

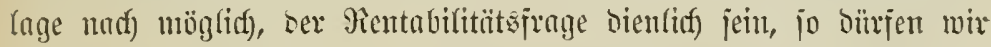
uidjt sie zu einer bejtimmten Zeit probujirte Nanje an uno fitr jicf

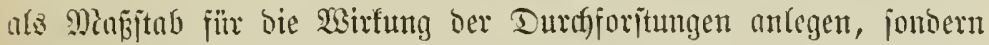
miijen audf) ber 2(rt ber 3umadfolagerung, wie jie ben 2 Sertl) bes

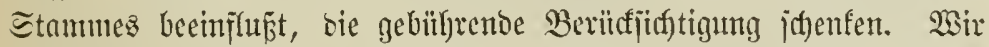

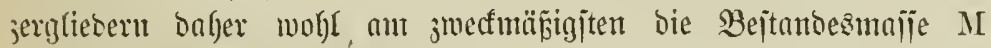

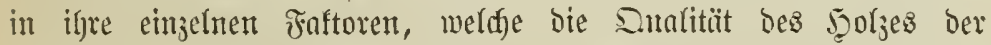

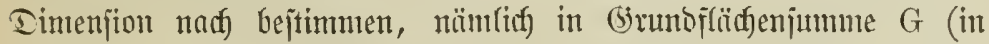

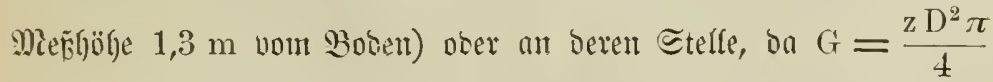

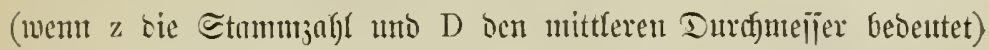
bie mittlere Bejtandesjtärfe, fermer bie mittlere Bejtandeshöhe $\mathrm{h}$ uno bie mittlere jormzahl f. Sdfliefilid) juj̄en wir bas firooutt Ghf $=$ M zm : jujanmen.

a. Beftantoesfärfe.

In jebent aud noff jo regetmï̈̈igen Bejtunde - uno jei er

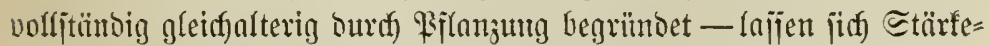
itujen unterja)eiden unto zmar in ber Megel um jo melor, je älter er 


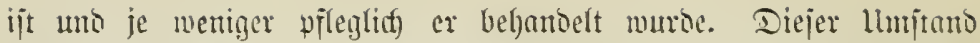

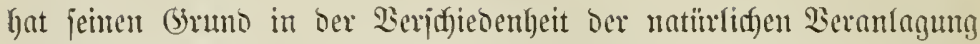

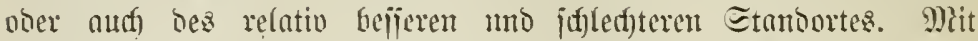

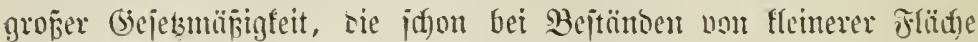

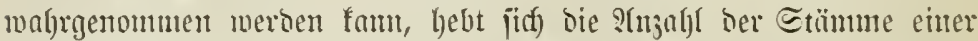

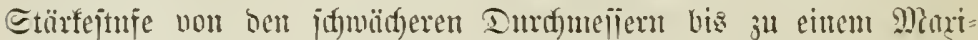

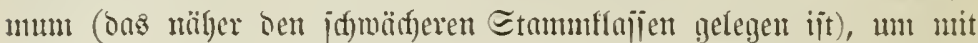
Bunahme bes Durdmmejiers mieber ju jüfen. Trägt man bie in

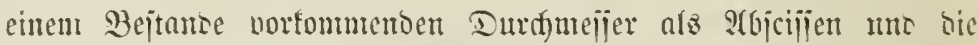

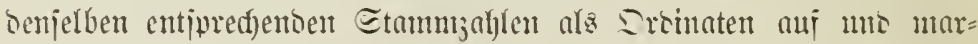
firt surd) cinen $3^{\mathrm{ug}}$ ans freicr Dome interpolationsmeije bie :age

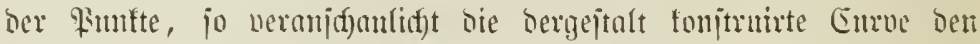

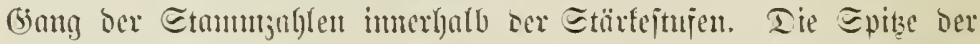

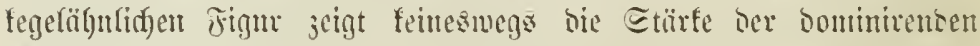

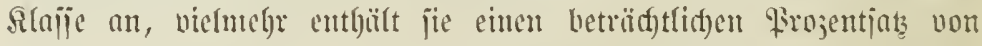

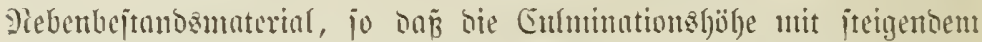

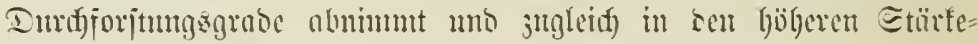
jtujen weiter ritift.

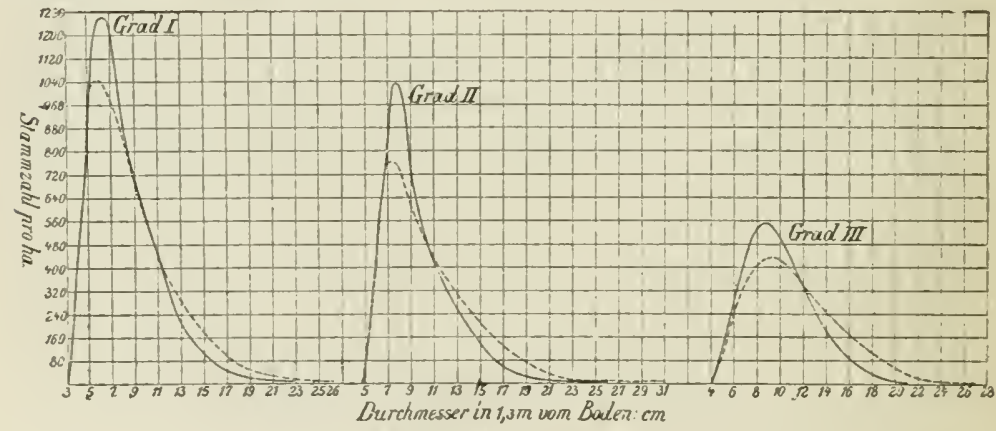

zrig. Ia: $\Xi a n t b e j t a r i$.

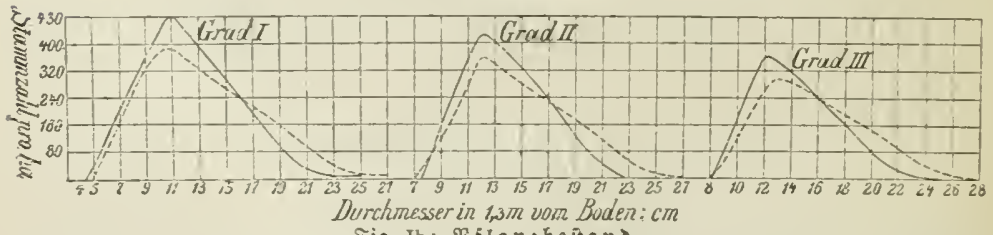

Frig. Ib: $\mathfrak{B}$ f I a n beitano.

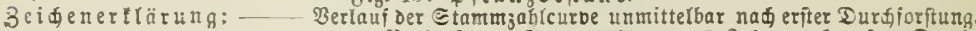
Berlauf Der Stammablcurbe 5 3abre naळ exjter $\mathfrak{D}$ ur jorftung. 


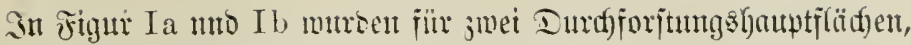
gelegen in fgl. Gayr. Foritamte Eadjemrieo, Dijtr. Eumjadf), $\mathfrak{2}(6=$

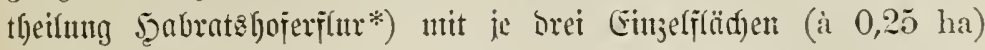

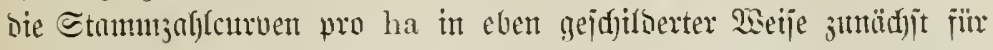
Den Stand unmitte(far nad) ber eriten Imrfiforitung oargejtellt.

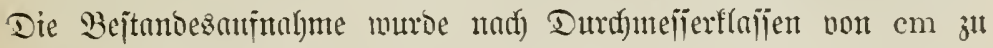

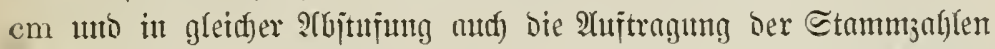

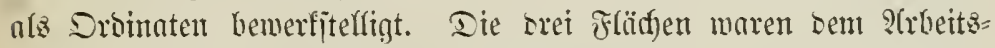

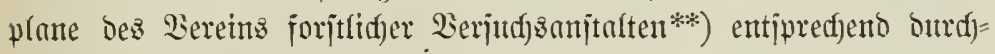

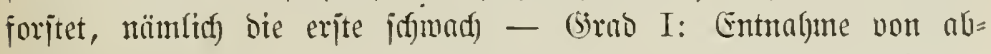
gejtorbenem uno abjtertentom Miatertafe —; bie jmeite mäß̈̈ig (5rad II: Entnalyme von unterbrïctent @tümmen -; bie britte

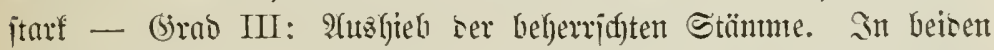

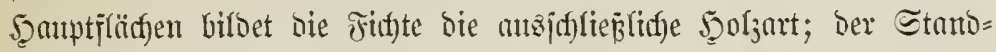

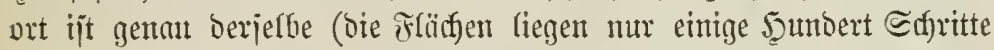
non einamber entfernt); jeoon ijt bie Begründungzant berjafieben, inbent bie eine - 38jägrig - burdg Eant, bie anbere - 37jälfrig -

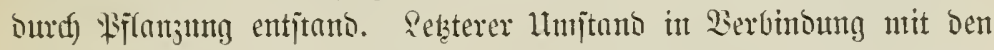

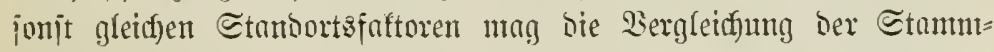

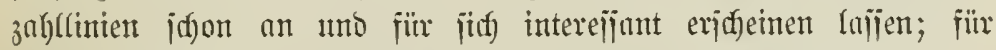
unjer Thenta ijt jeood) ipejiell bie Frage non wistgtigfeit: In

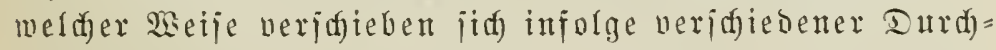
forjung nad) einem, gemijien Beitrante bie Etammjablen innergalb ser Etärfejtujen ober anders ausgebrïft: Wiste gejtaltet jidf ber Zumadjz ber einzelnen Stammiärlen?

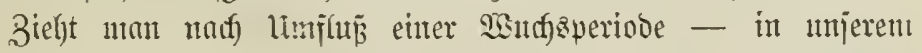

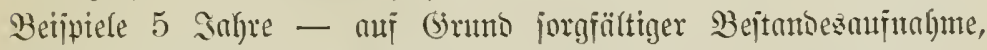

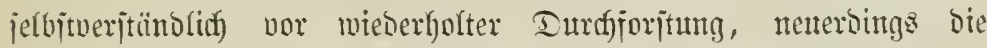

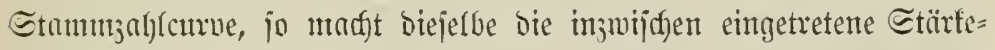

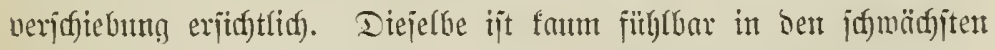
Stammiflajent bes Wolfbejtances ober in (Grad I, bagegen idjont be= trä̈thtidid) in (brab III, in weld) leţterem bie punftirte Sinie ber

*) Der betreffende Beitano fitoct $780 \mathrm{~m}$ God ïber bem Meere auj ebenem

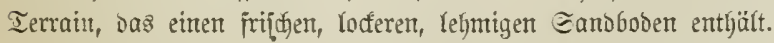

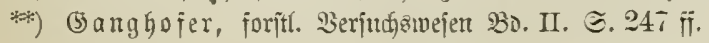




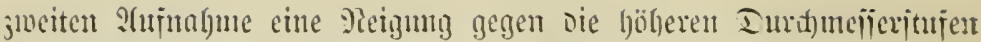

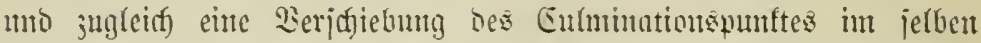

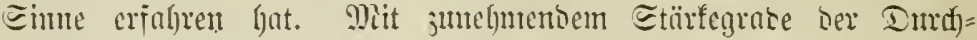

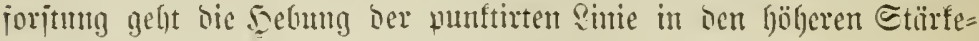

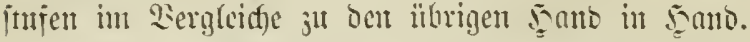

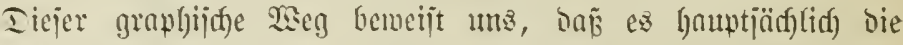

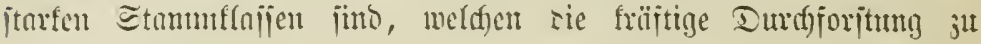
ginte fommit.

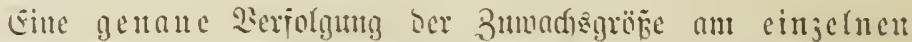

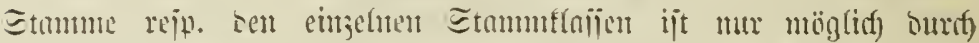
Petmerinuty berjelben. Iiejes Frincin verjolgt ber santon= Sber=

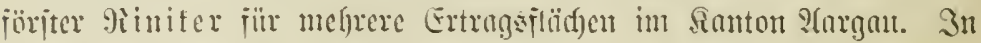
belïen Edfriftdfen*), Ier Zumadfagang in jidftent= unt Budfen=

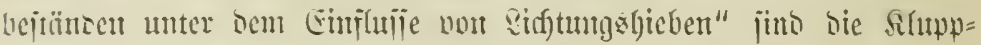
mannale nad) cinjschten mmerinten Etämmen fïn bie 2(ujnalnten in

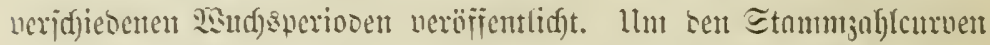
jüntgerer Bejtünto ente joldfe älteren Bejtanocs entgegenjtellen uno

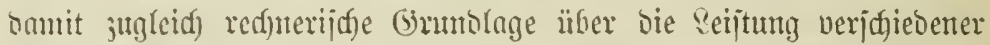
Etanmflnijen gemimten jt fömnet, judjen mir bie 2trgaben bes

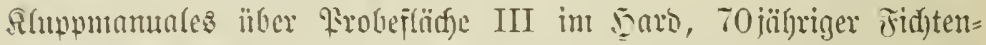

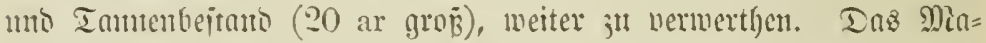
unal bictet bie Etänme (1.3 Etild), wie jie in ber 9intur neben= einnter jtehent ammerirt unt auf $\mathrm{mm}$ genta gemejīen murben.

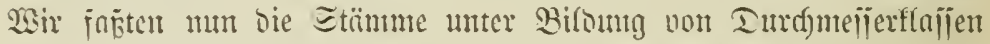
von je $3 \mathrm{~cm}$ (wic $13,0 \mathrm{~cm}$ bis 15,$9 ; 16,0$ his $18,9 \mathrm{~cm}$ u. i. i.) jujammen, Gereffneten fïr iebe Ilajie sas Mittel ber Miejungen

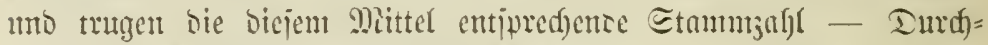

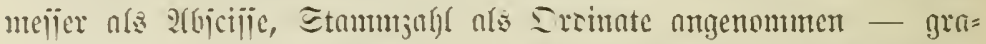

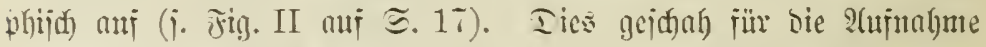
unmittelbar nad) bem ?hushiebe vout $7,5 \%$ ber norlhanbenten Stanm=

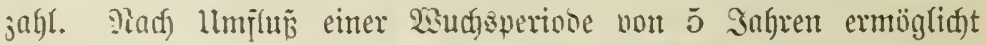
bie Tiumerirung oer eimjelnen હtänme, bic Slaijen mit ben nüm=

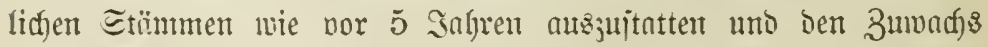
biejer Slaijen jut ermitteln. Fitr bie beredgneten nenen Durdf =

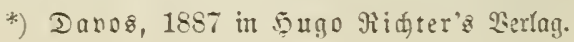




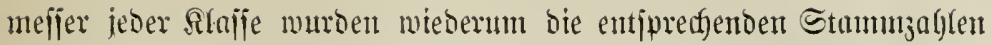

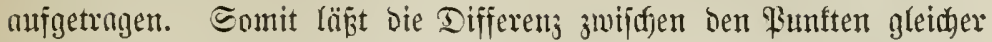

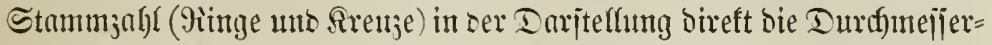

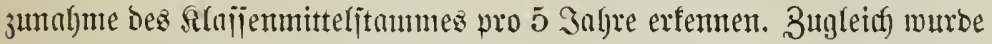

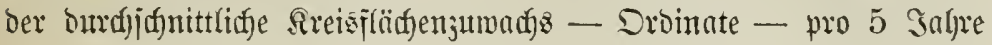

Fig. II.

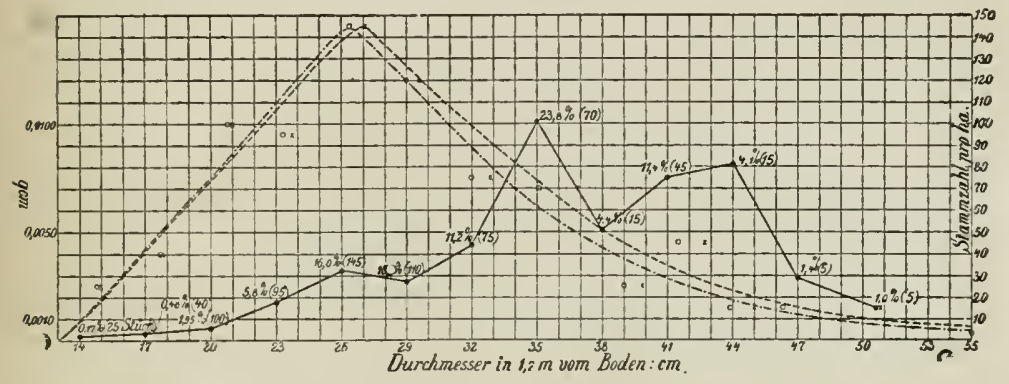

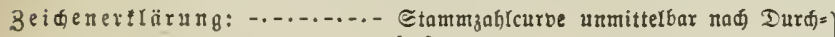
forftung.

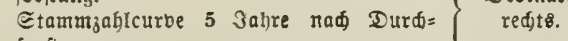

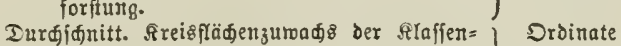
mittelftämme in 5 3ahren.

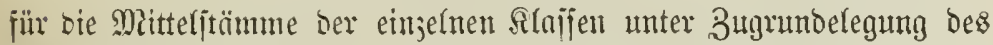

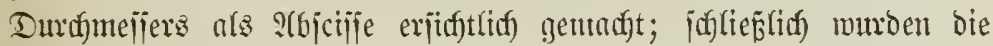

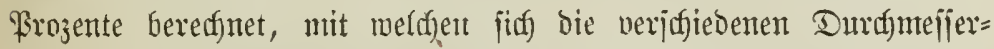
jtufen an gejammten (srunbflïgenjumadje betbeiligten. Die ge=

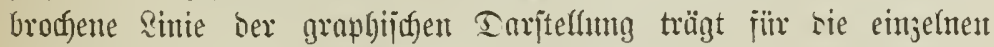
Durfmeljeritujen von 3 ju $3 \mathrm{~cm}$ die $2(n j a g l$ der Rrojente, mit meldfer fith rie in Slammer beigejetzte Stammjagl ber betreffenden

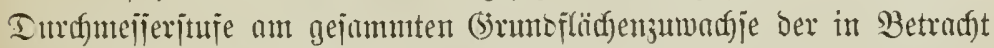

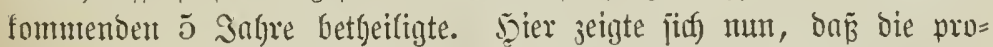

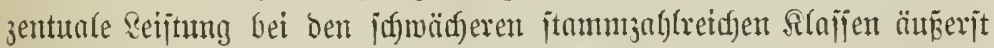
gering ijt, bã mejiers jtattfintet bis ju einem Cutminationspunfte unb bam wieber Sinfen eintritt. Die Gulmination fiel bei einem Durffmefferipatium non 10-69 $\mathrm{cm}$ Der Silnije $35 \mathrm{~cm}$ bei mu $10 \%$ Der Stamnizahl mit $23,8 \%$ ber (5ejanmtreijtung 31t. $34,6 \%$ Der (5) jammt= jtammjahl, ben jamäderen alajen angehörend, bat nut 
$8,5 \%$ oer (5ejummtle ifund, ongegen $65,4 \%$ ber (jejumt=

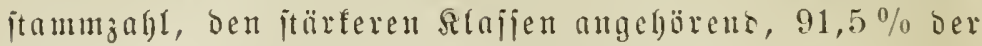
(3) ejammteijtung an brunbilïdenjumads anjumeijen. Somit muren von 800 Stünmen pro ha, trotzocm cin ?hasfieb von

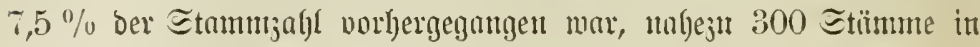
jef) yeringem Miaje junadysthätin.

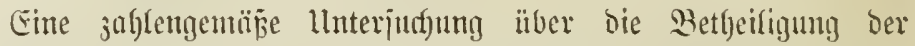
Stïrfeffajien an Bejtanowzumadje jtelfte Dr. (Brmoner an*).

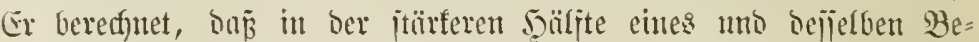

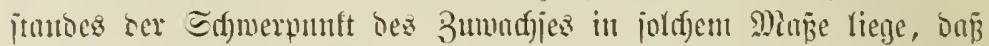

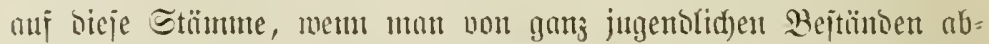
juge, menigitens $80 \%$ ses (sejunumtzumadjjes entfolle. Bum gleiffen

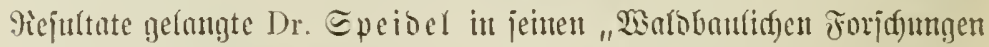

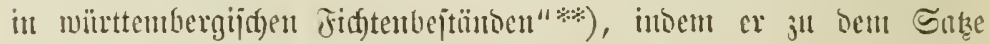

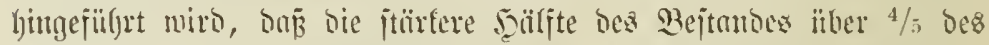

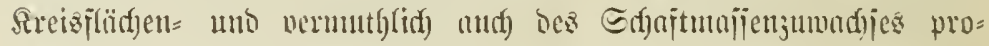
Dujite***).

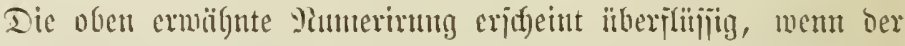
Erjolg einer :

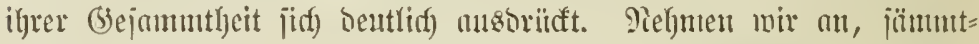

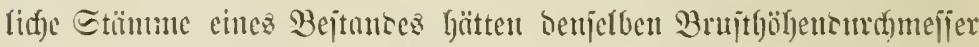

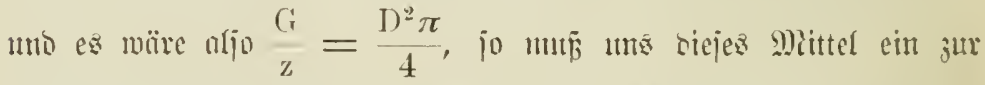

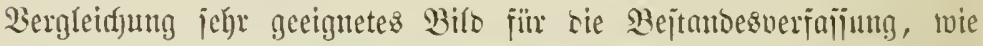
jie surd) Den verjofiedenartigen Eingriff umutittelbar uno mittelbar

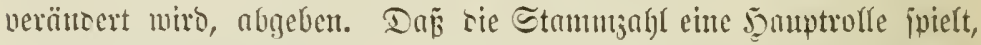
ijt gunj jelbjtneritündlid). Diejelbe mus theoretijd) fïr bie jujammen=

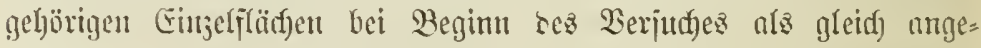
nommen werben; praftija) sagegen ijt bie vöffige llebereinjtinmmung

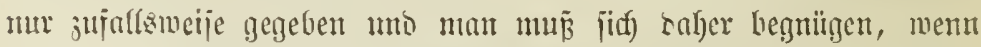

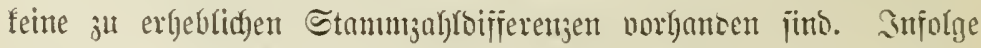
erfmaliger Durdfjorjtung zeigen bie mipringlidf) gleidfen Mlittel=

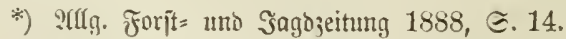

**) Tübingen, 1889, ๔. 49 .

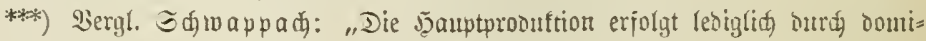

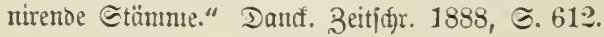


Surdmeljer ser Gingelflüd)en Difjerengen, die jür ben Grab ser Durffforitumg (barafterijtijd) jint. Se jtïrfer niumlidf ber Sieb bas Tiebenbejtanosmateriar rebujirt, is meiter man ren Begrifi Les

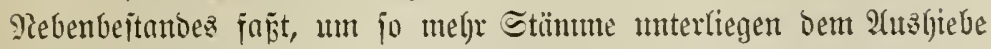
uno un fo boüger bebt jid) mit Sinfen uon z ber aritgmetija) mittlere Durfumejier. Sir Gaben baher bei der für eine Durfforjutungs:

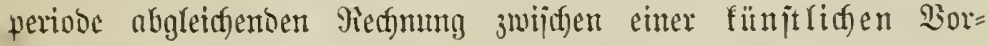

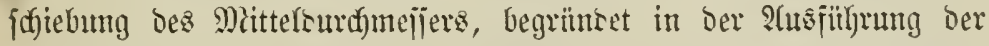

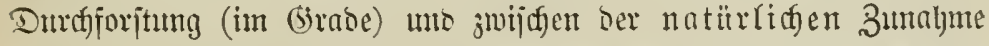
befïelben auj (5rumb gejteigerten 3umadjes zu unteridgeioen. Erjtere ijt bis ju ciner gemiljen Grenje sie Urjadje ber Yetzteren.

Bleiben wir bei ben jfjon angeiüljten Beijplelen bes Forjt= antes Sadjenried!

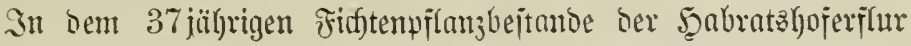

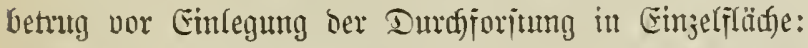

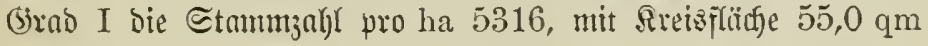

$$
\begin{aligned}
& =\text { II }==5252=56,6= \\
& \text { III }==5110=53,3=
\end{aligned}
$$

Die erjte Durdforitung entnalym ats

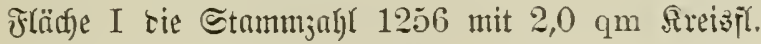

$$
\begin{aligned}
& =\text { II }=\quad=2056=6,3== \\
& \text { = III }=\quad 2428=8,4=
\end{aligned}
$$

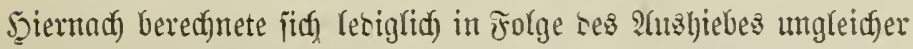
Stanmjahlen uno Stärfen ber arithntetijule Mittelouramelier fïr Fläd)e I ח1ז $128 \mathrm{~mm}$

$$
\begin{aligned}
& =\text { II }=142= \\
& =\text { III }=146=
\end{aligned}
$$

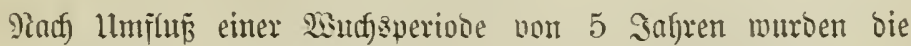

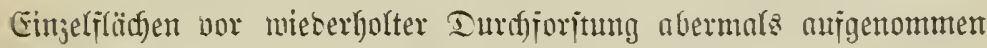

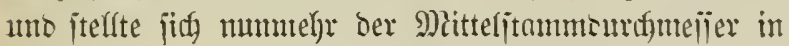

Fläcye I auf $137 \mathrm{~mm}$
$=$ II $=154=$
$=$ III $=160=$

Sonady war ber Mitteljtamm in

$$
\text { Fliicf)e I um } 9 \mathrm{~mm}
$$

$=$ II $=12=$
$=$ III $=14=$


in 5 Sabren jugemadjen, moraus hervorgeft, baj ber Mittelitanum von Fläd)e II gegeniüter bem von I um $3 \mathrm{~mm}$ mo frädge III gegenüber sem uon I $\mathrm{mm} 5 \mathrm{~mm}$ in derfertben Beit mefr an Stärfe zugenomment ljat.

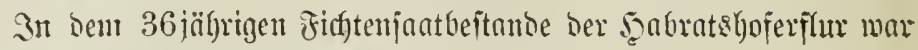
vor Beginn res 2erjutfes in

Flïd) I bie Etunmughl 19268 ; bie Rreisiflädjenjumme $49,9 \mathrm{qm}$

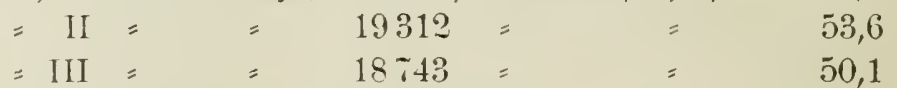

Die Turdjprituntg entutalun ans:

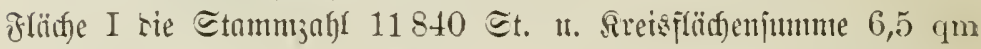

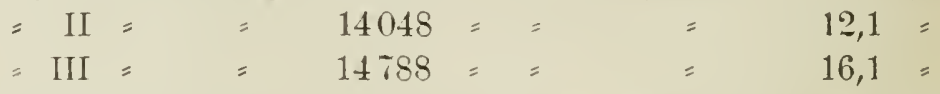

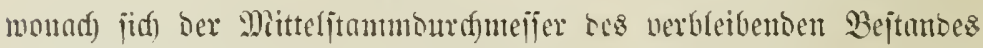
beredfute in

$$
\begin{aligned}
& \text { Ffläche I aนๆ } 86 \mathrm{~mm} \\
& =\text { II }=100= \\
& =\text { III }=105=
\end{aligned}
$$

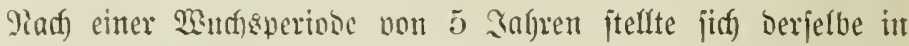
$\left.\begin{array}{l}\text { Fläd)e I mif } 97 \mathrm{~mm} \\ =\text { II }=112= \\ =\text { III }=118=\end{array}\right\} \begin{gathered}\text { jontud) Zuntume } \\ \text { in } 5 \text { Safrett }\end{gathered}\left\{\begin{array}{l}11 \mathrm{~mm} \\ 12= \\ 13=\end{array}\right.$

unto bie thatjädflifye Metyrlejitung gegenüber (5rab I bei (5rab II anf 1 uno hei brab III auj $2 \mathrm{~mm}$ in Ber Etärfe.

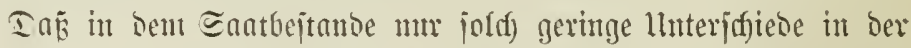

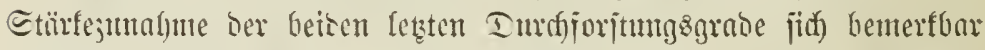

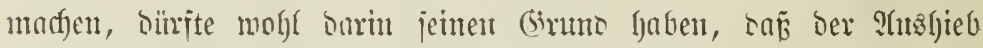
Des abjtüntigen Miatertales in (jrad I aud bem bisher viel ju bicften Bejunte unu mejentfidjen Bortfeilc mar, ber eben bie

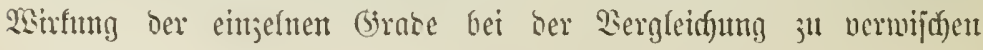
geeignet ijt.

?(ngenonmen, jänuntlid)e Etämme in einem Bejtante rätten im

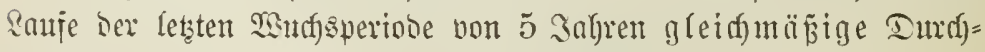
mejierzunagme erfafrent, jo würoen bie Etämme bes:

Fiflanjbeitantes in Fr. II am je $0,6 \mathrm{~mm}$; in Fl. III um ie $1 \mathrm{~mm}$ Enathejtanoes $==$ II $=0,2=\quad=$ III $=0,4=$ 
Wro Sangr an Etürfe gegenitber Denen non Grad I noer bes Soufs Kejtanbes jugenommen labent.

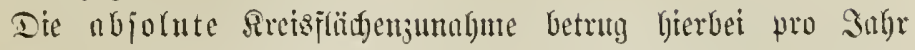
tuto ha:

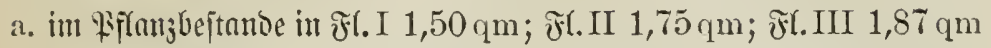
b. $=$ Snatbejtonoe $==$ I $2,02==$ II $1,98==$ III $1,87=$

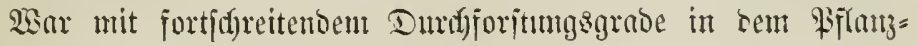
bejtanoe, ser won fritgejter Sugeno an lisfter gejtefit war, bie abjo=

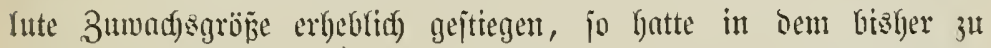
georïngten Snatbejtanoe bie Durdjoriftung sie untgefeffrte Wsirfung

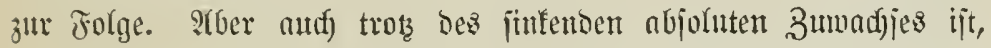

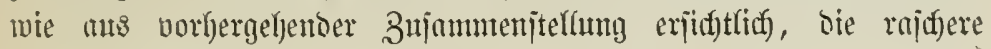

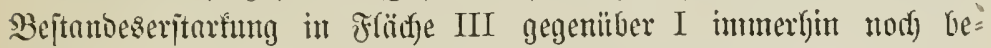

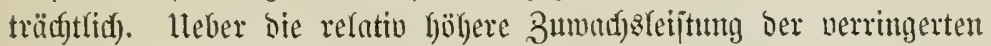

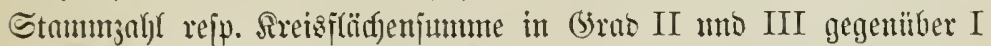

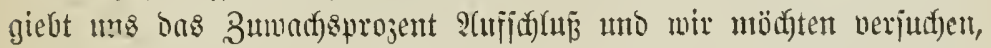

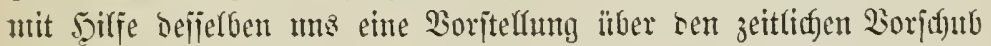
ber Sintzung, welder ber rajderen Bejtandeseritarfung entipridjt,

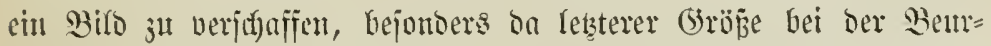
theilung ber Pientubifitïtsfrage eine nidgt unbebentente giolle jut= fommen bilrite.

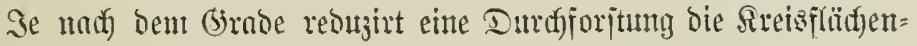
jumme (bejogen auf $1,3 \mathrm{~m}$ 'vom Bocen) Des betreffenten Bejtandes

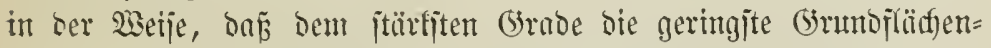
fumme verbleibt. Waitros nad) einem genifien Froduftionsjeitrume

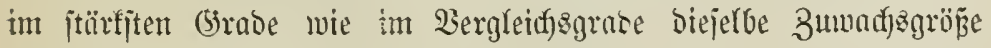
erjengt, fo müre bie Seijtung feinesmegs auth) eine gleiffe, viefmethr eine um jo höhere, je geringer sie produjirente Stmme mar. Lleber

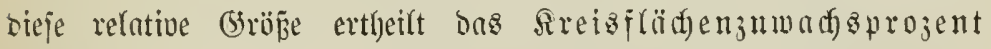

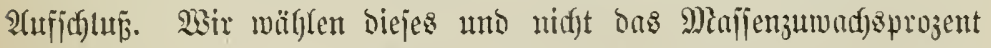

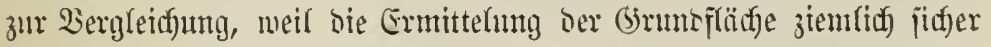

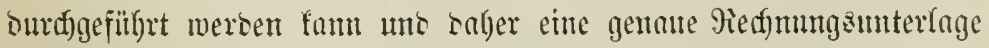
genüllyt, fermer weil wir ljier ipejtell bie Etärtejunalgme bei ver= fidjedenten Durtfjurfungagraben verfolgen mollen.

Es bejeidyne g bie Sreisflärfe eines Beptandes zut Beit des

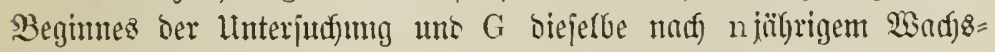




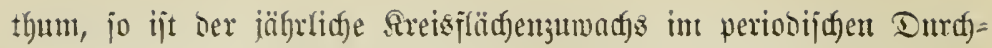

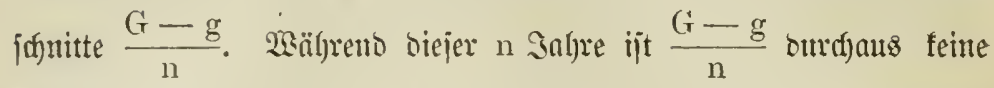

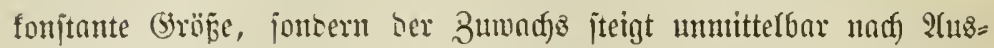
füfruutg ber Turdjoritung an uno nimmt mit ber $2 e r s i d$ tung bes Bejtntessidfuijes mieserum ab. (Es fann baher weter g nod) bas

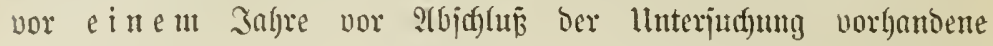

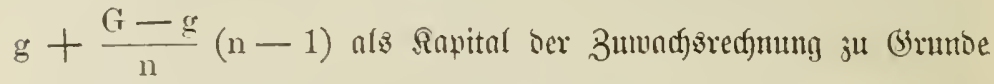
gelegt merben, jonbern sas Mittel jwijdjen siejen beisen Extrement, nämlidy bas in Ser Mitte ser F'eriobe tyätige Sapital miro an bejten

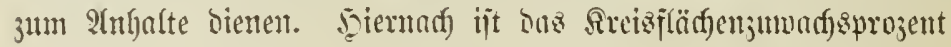

$$
\begin{aligned}
& \mathrm{p}=\frac{\frac{(\mathrm{i}-\mathrm{g}}{\mathrm{n}}}{\frac{\mathrm{g}+\frac{(\mathrm{i}-\mathrm{g}}{\mathrm{n}}(\mathrm{n}+1)+\mathrm{g}}{2 \cdot 100}} \text { ocer } \\
& \mathrm{p}=\frac{200(\mathrm{G}-\mathrm{g})}{\mathrm{G}(\mathrm{n}-1)+\mathrm{g}(\mathrm{n}+1)} .
\end{aligned}
$$

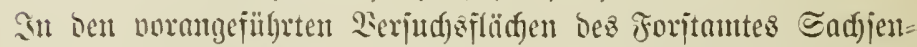
rieb mar, menn bie Rreişlädyenjunmen ber einjelnent flädgen uns Deren Buwadjôprojente burd) set Intę ber betreffenden Durdf $=$ forjtungegrabe gefennjeifnet werten:

a. Für sen Yiflamjhejtano

$$
\begin{array}{ll}
\mathrm{G}_{\mathrm{I}}=60,53 \mathrm{qm}, & \mathrm{g}_{\mathrm{I}}=53,01 \mathrm{q}_{1} \mathrm{~m} \\
\mathrm{G}_{\mathrm{II}}=59,39= & \mathrm{g}_{\mathrm{II}}=50,65= \\
\mathrm{G}_{\mathrm{III}}=54,29 \Rightarrow & \mathrm{g}_{\mathrm{III}}=44,92=
\end{array}
$$

uno mit ñife obiger Formel beredfnet jidf jür bie $2 \mathfrak{s u d j} 3=$ pertose nou 5 safren

$$
\begin{aligned}
& p_{\text {I }} \text { nui } 2,68 \% \\
& p_{\text {II }}=3,22 \% \\
& P_{\text {III }}=3,85 \% ;
\end{aligned}
$$

b. Fiir den ভantbejtano mar

$$
\begin{aligned}
& \mathrm{G}_{\mathrm{r}_{1}}=53,42, \quad \mathrm{~g}_{\mathrm{I}}=43,33 \text { unt liernadi } \mathrm{p}_{\mathrm{I}}=4,27 \\
& \mathrm{G}_{\mathrm{II}}=51,41, \quad \mathrm{~g}_{\mathrm{II}}=41,50=\quad=\quad \mathrm{p}_{\mathrm{II}}=4,39 \\
& \mathrm{G}_{\mathrm{III}}=43,29, \quad \mathrm{~g}_{\mathrm{III}}=33,92=\quad=\mathrm{p}_{\mathrm{III}}=5,02 \text {. }
\end{aligned}
$$


Ser 94tabieb gatte betragen:

$$
\begin{aligned}
& \text { im im } \\
& \text { Hifanjeftance Earbeftande } \\
& \text { Fläxfe II } 5 \% \quad 4 \% \text { । der uriprïngliffen } \\
& =\text { III } 16 \% \quad 22 \% \text { (5itmbiliës)e. }
\end{aligned}
$$

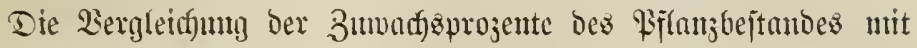

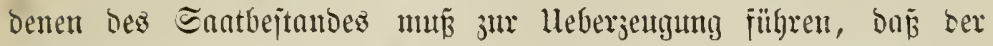

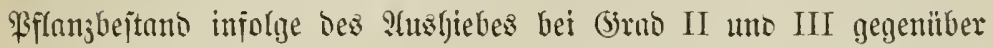
(3riab I mebr geleijtet hat rejp. rajuer exjtarft ijt als bie gleiffen Flänen bes @antbeitumbes.

Es liegt aljo bier eme Berjufebung in ber Beit ber Piutzung

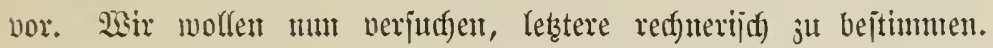

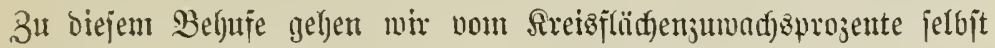

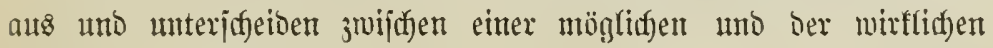
Reiptuny eines Beitandes.

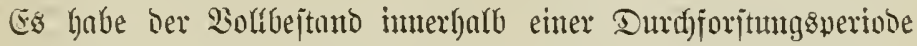
von $n$ Sabren jeine Sreisflüd) $g$ auf $G$ geboben uno ljierbei mit

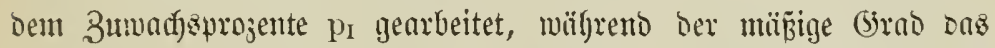

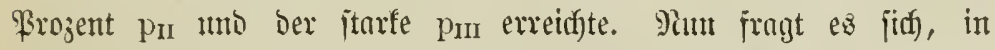

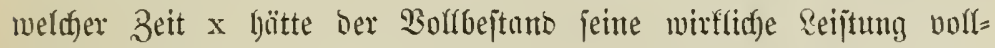

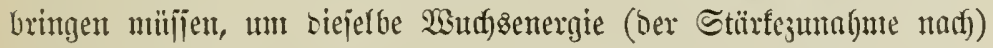
wie (5rao II ober Grad III jat entĩalten. Sifentur mur bieje Zcit

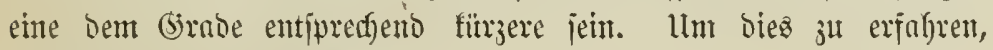
bentuten wir bie (3)leidfungen:

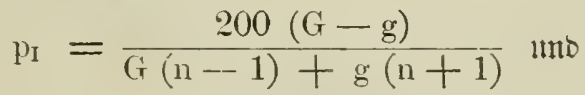

$$
\begin{aligned}
& \mathrm{P}_{\mathrm{II}}(\mathrm{III})=\frac{200(\mathrm{G}-\mathrm{g})}{\mathrm{G}_{\mathrm{r}}(\mathrm{x}-1)+\mathrm{g}(\mathrm{x}+1)^{\prime}}
\end{aligned}
$$

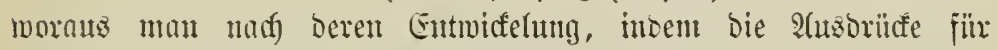
$200(\mathrm{G}-\mathrm{g})$ eintancer gleiffgejełzt werben, findet

$$
\mathrm{x}=\frac{\mathrm{p}_{\mathrm{I}}}{\mathrm{p}_{\mathrm{II}_{(\mathrm{III})}}} \cdot \mathrm{n}+\frac{(\mathrm{G}-\mathrm{g})\left(\mathrm{p}_{\mathrm{II}_{(\mathrm{III})}}-\mathrm{p}_{\mathrm{I}}\right)}{(\mathrm{G}+\mathrm{g}) \mathrm{p}_{\mathrm{II}_{(\mathrm{III})}}}
$$

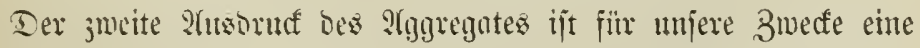

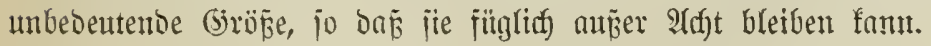

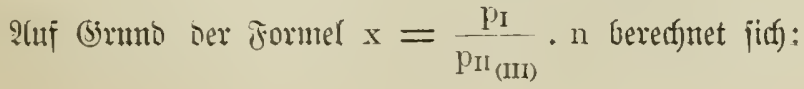


a. Fïr ben Piflanjbejtano:

bei Fläche I gegenituer II $\mathrm{x}=0,84 \times 5=4,2$ Salfre

$=$ III $x=0,71 \times 5=3,6=$;

b. F̈̈r cen Eantbejtans:

bei Frläge I gegenilher II $x=0,97 \times 5=4,9$ Intre

III $\mathrm{x}=0,86 \times 5=4,3=$;

bas beipt aljo, wenn wir ben exiten Fall ins शruge fajien, Grob I

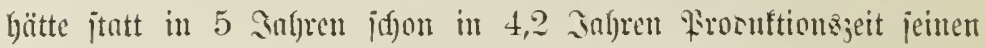

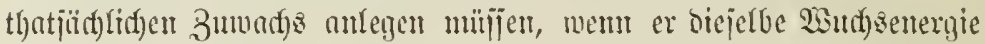

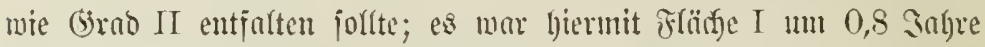

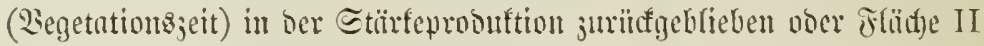
un ben nïmlidjen Betrng ber Jenubarfeit nïler gerïat.

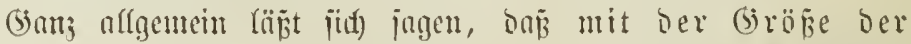

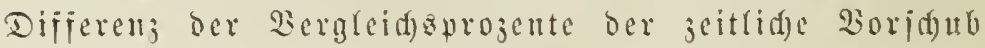
ber Mutzontefeit wäd)jt.

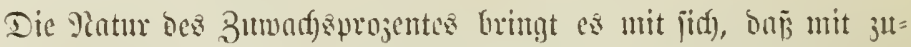
neljmentem Bejtandesalter, jelbjt gleidfbleibencen ober nod jteigenten

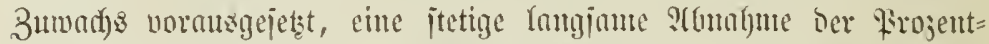
ziffer eintritt, intem das fixe arbeitenoe Sapital - ment nicft jtörento Eingrifïe gemad)t merben - im Bejtunde jid) in bem Mraje bäujt, als bie Beit ber sombarfeit näher rïft; bagegen bie prosujirte

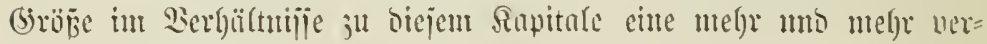
fifmumbente mirt.

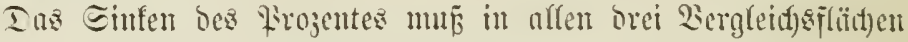
eintreten, jeooch wiro, menn man in flüchen nit (5rat II uno III

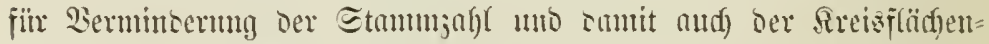

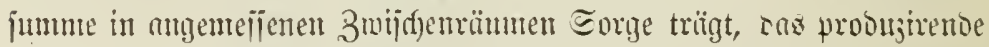

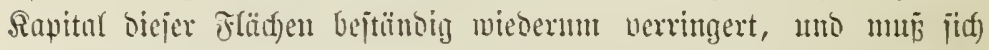

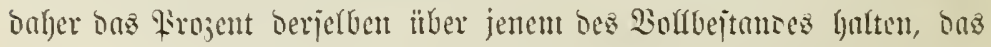

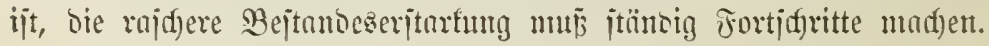

Iiez ijt aud) ofne meiteres flar, menn man ermägt, bas bei bem abgentuberten Sapitule cine verhältnip̈niäßing geringe Etanmtgnhl

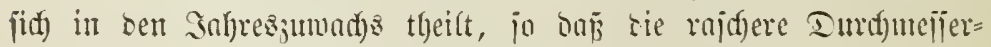

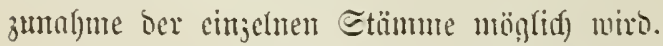

ltm ein fontrajtivenoes Beijpicl anjinteffen, nelumen wir an, es

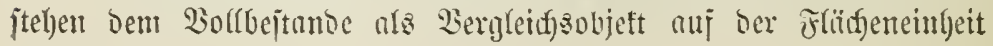


nur vereingelte in völfigem Fretitanto Gefinolitfe Etänme gleidfen

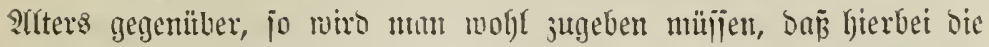
Difieren; der Zumadfsprojente gegenitber sen anderen fällfen ein

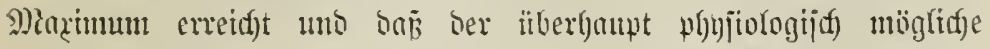
Borjajub in ber Durdumejererjtorfung erfolgt. S(noererjeits jeigt biejes abjtratte Beijpiel and, san bie böbere abjolute Proouftion

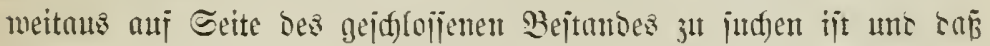

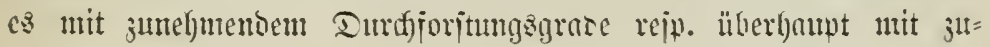

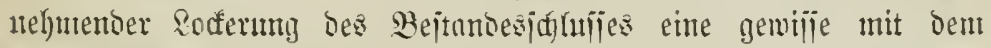
Bejtandesalter medjeltnte birenje gebent mus, bis ju meldjer ber S(ushieb sie Störtfejunafjute förtern fam, ofjne jugletif) bie siermin=

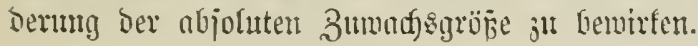

\section{b. Eä̆he mo Stammiorm.}

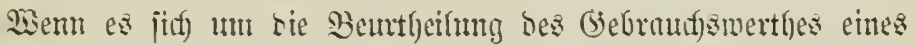

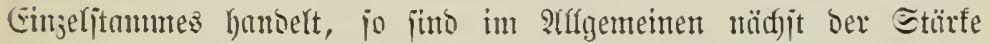

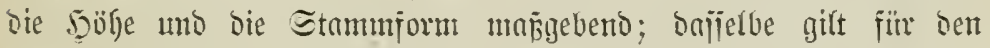
ganjen Bejtant. Daraus geljt bie holje Bebeutung herwor, weldje

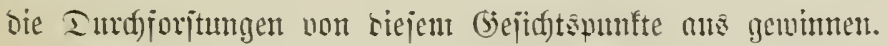

Mian jolfte glanben, ba cin Bergleidf) ber aritfmetija mittleren

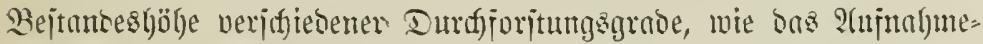

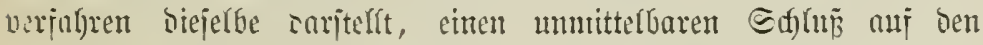

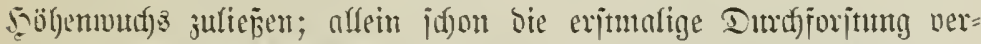

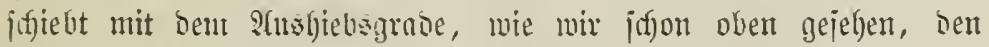

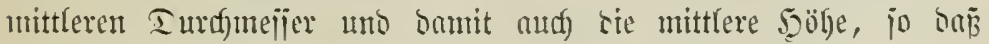

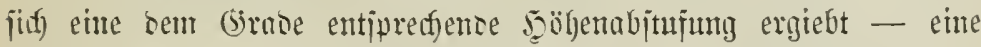

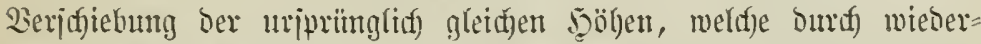

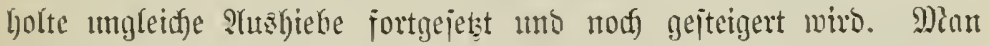

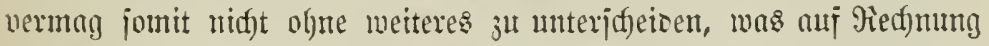

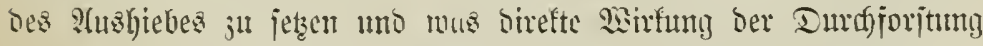

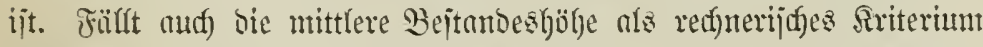

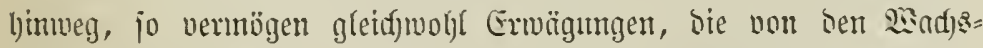
thum

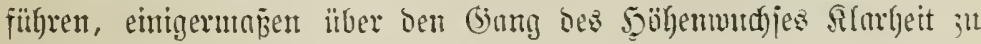
erthalten. 


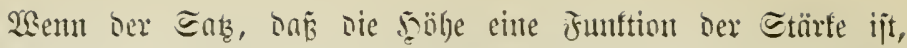

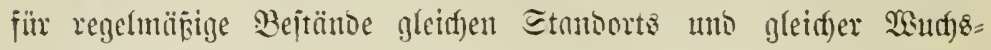
bebingungen als giltig angenommen merben bur - mo bie Er= fahrung hat bics liskger hejtätigt - jo jofgert für Bejtünte ver=

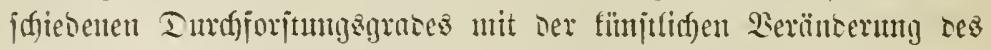

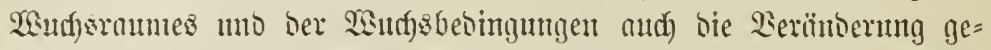

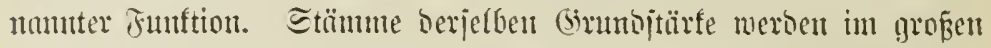

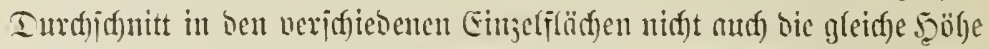

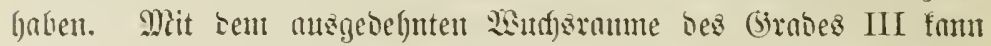

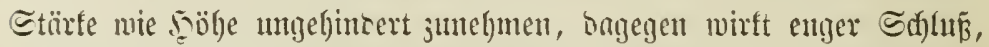

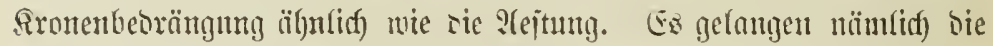
Bif(sungsitofie nidgt in benjelben Mañe nad) ben unteren હtanun=

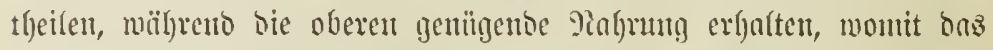

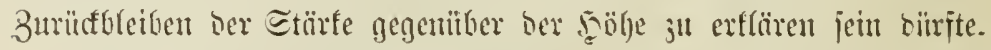

Mit bicjem serbalten ijt jugleing nud bie Stammjorm bei verjofiebenemt Édfluggrabe besingt uno gegeben. Ier fichtere Etano ver=

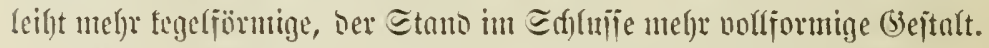

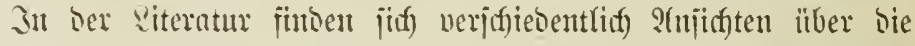

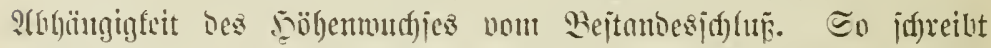

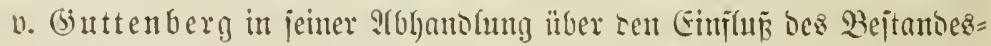

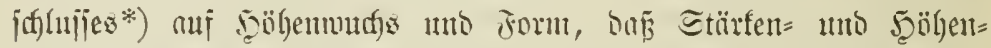

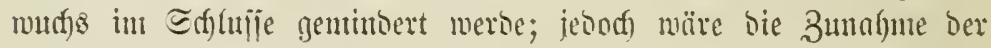

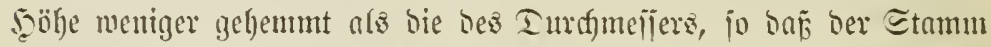
eine föblere uno joffanfere form erlyalte.

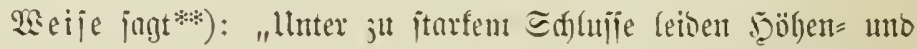

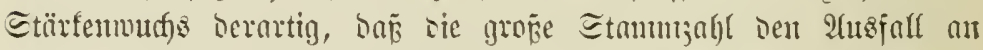

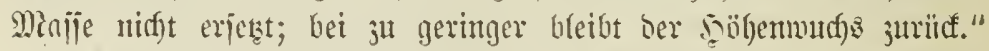

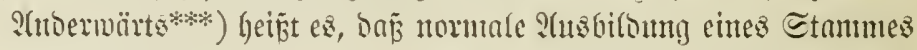
normaten sisadistan erforsere; cine serringerung befielben ver=

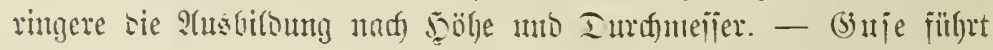

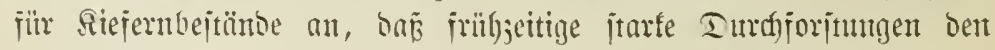

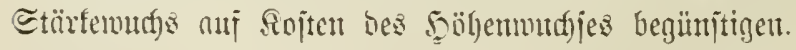

\footnotetext{
*) Septerr. 2ierteliahräđarit 1886, p. 103.

**) Seije, Chroutif 1881, E. 25.

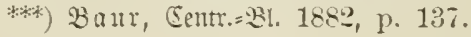




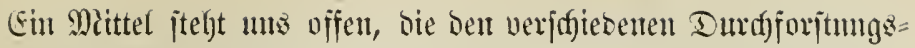

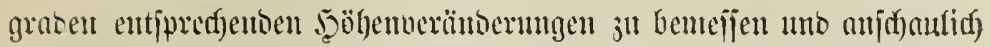

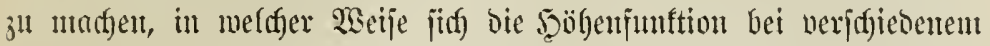

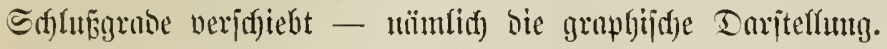

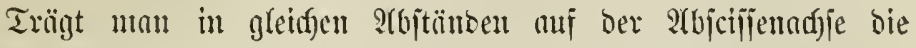
Durdutufierifufen, melife in bem ju unterjutfenten Bejtanoe nor=

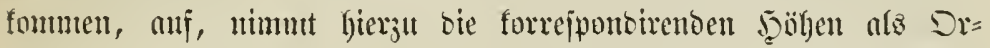

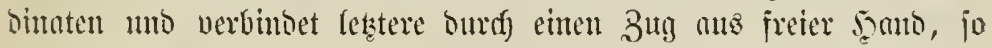

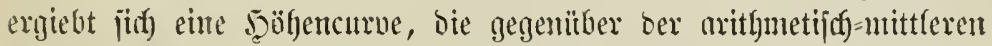

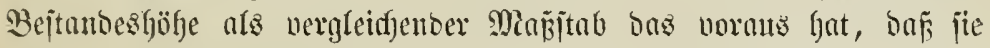

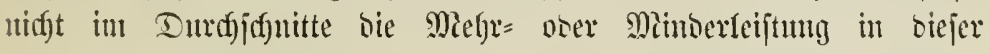

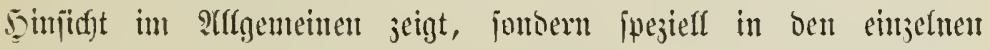

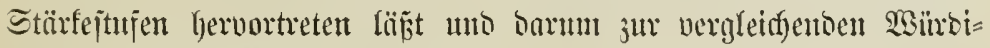
gutry um jo ivertfooffer erjofint.

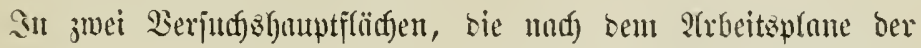

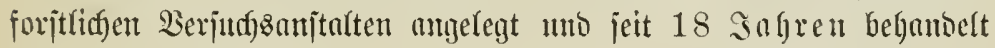

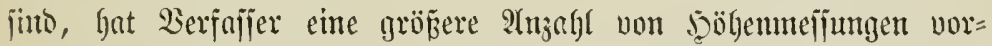

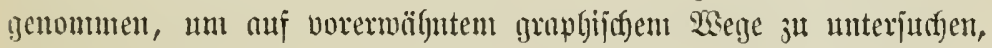
Fig. III a.

Fig. III b.

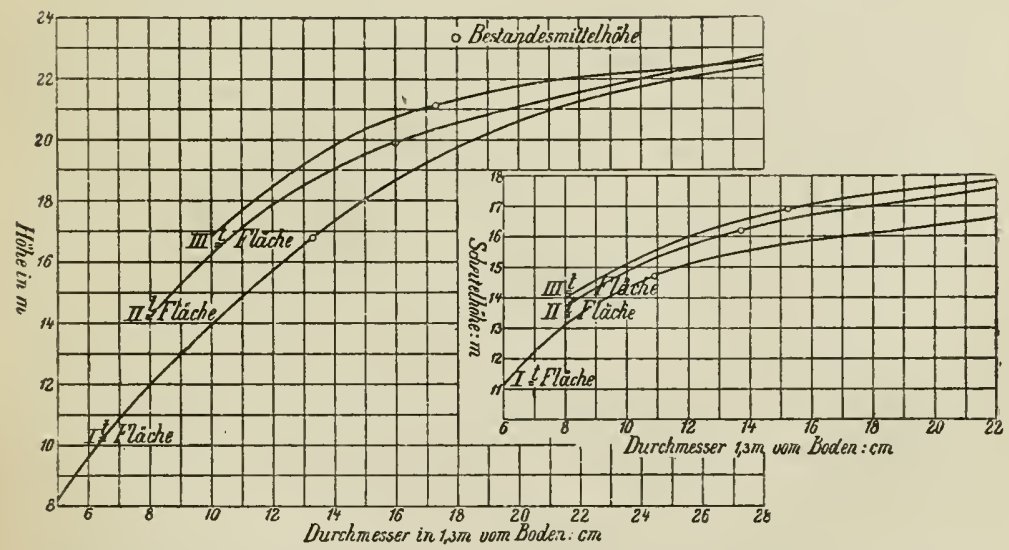

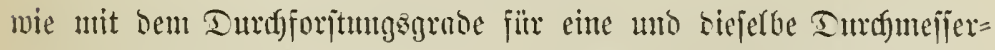

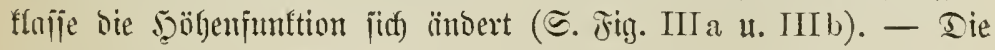

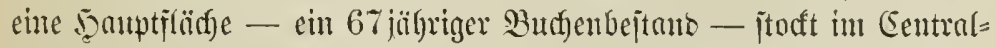
gebiete tes Eteigermaloes (Foritant Fathifjthleidfan) auj Boden 
befter Bonitüt (Reuperfanf). Die Stunbortzbeidgaffenteit ift in ben

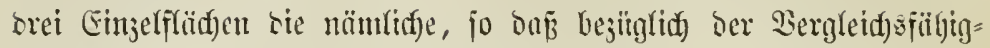

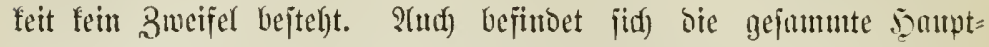

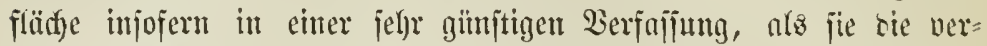

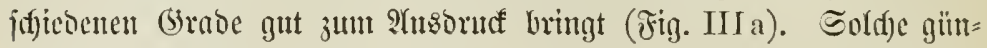

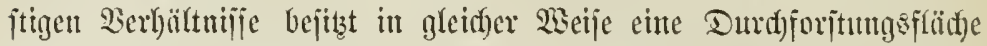
befteheno aus 46jülyrigen Riefern des Jantmoormaloes (Foritant

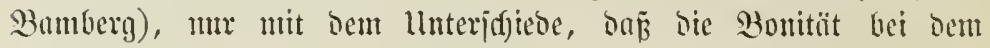
ansgeiprodyenen armen Sanbboden eine ben vorigen Flädjen ent= gegengejekzte ijt (Fig. IIIb). Trozzoent aber - uno bies neroient

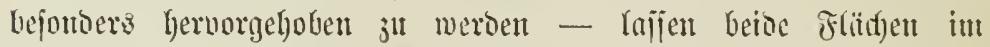

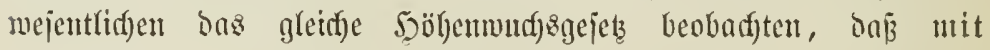
gejteigertem Durdjoritungsgabe die Döhenentmidelung ber Durdemefierzungute ganj betrïdtlid) uoratseilt, onz aljo wenigitens mälgreno eines gewijien Bejtandesalters

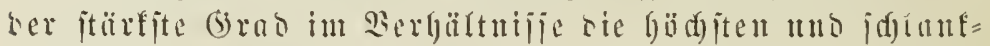
jteu Etïume erjengt.

Die beigefïnten Dingramme Injlen bet jeber filidge bie llnter=

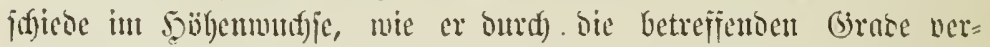

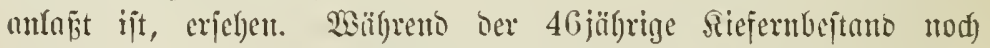

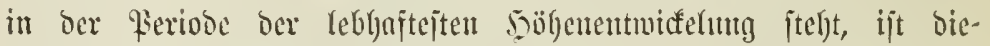

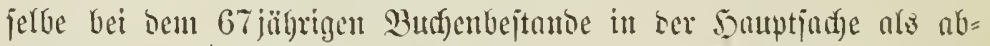

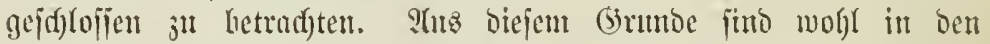

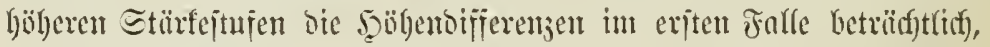

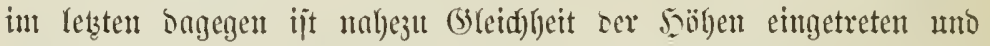

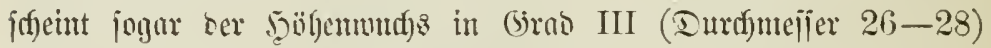

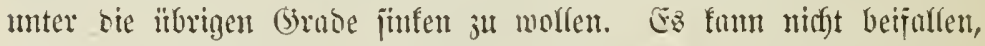

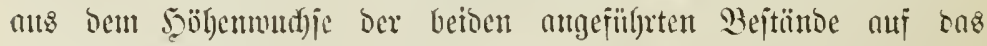

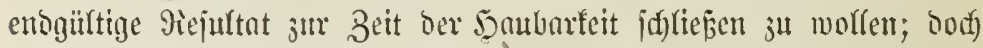

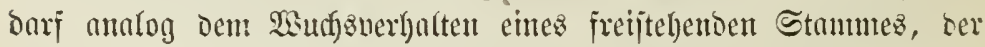

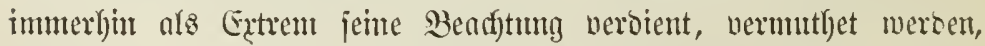

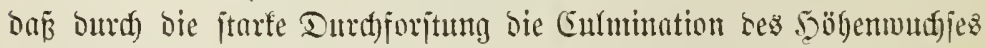
ebenjo wie bie Stärfeprobuftion ïberlyant zeitlidf) vorgej(f)ben wiro

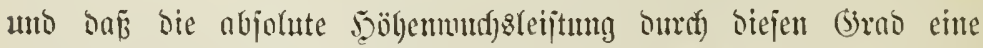
Berminderung erfalfren

Die mittlere Bejtanoesformzaly, jet es Sefjaft=, Derbl = ober 


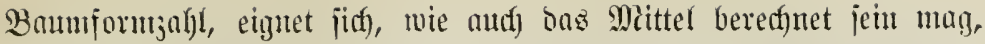

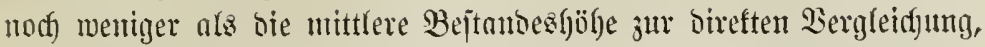
um ïber bie formberïntorung gleich alter aber neriffieden befan=

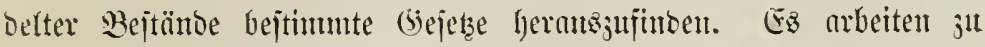
mnmigfadje Faftoren jul beren Bejtinmung jufammen, jo bas feime

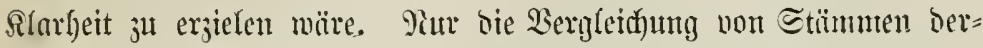

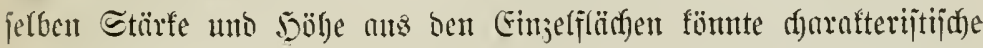

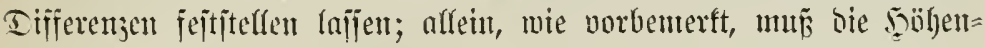

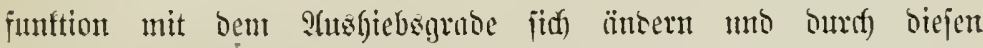

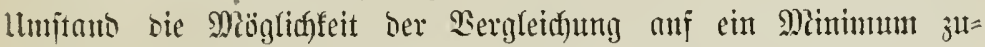

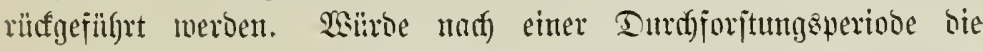

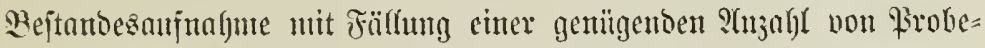

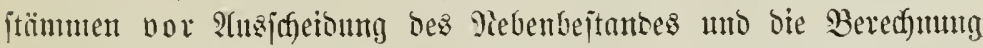

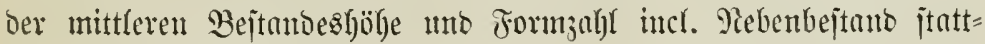

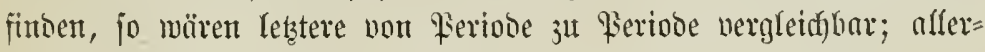

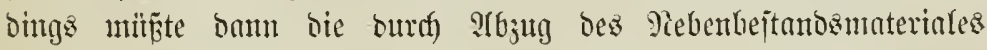

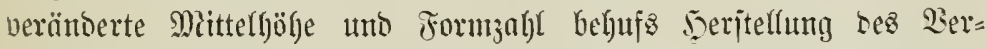

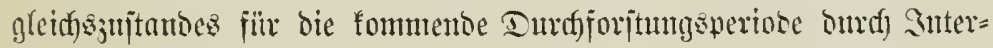
polation gemomen weroen.

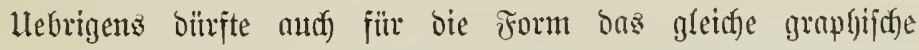
Berfafren wie bei ber Bejtantosfö̈ge 2(nmentung finden unb von Erfolg begleitet fein; namentlidf) wern bafjelbe jugleich mit ber

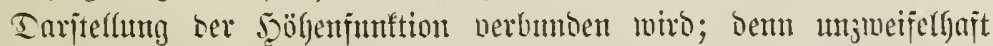

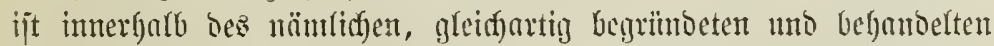

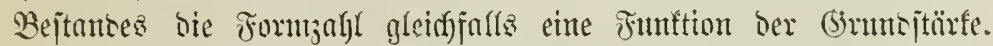

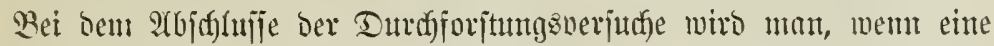

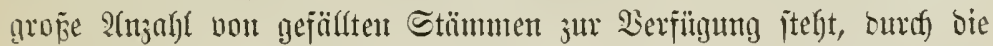

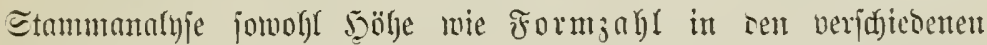

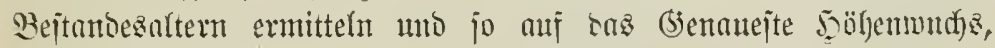

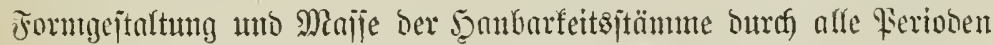

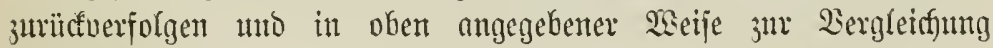
jiefen fönten.

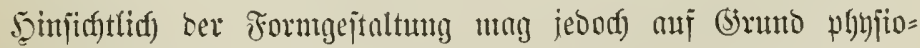

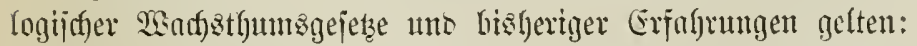

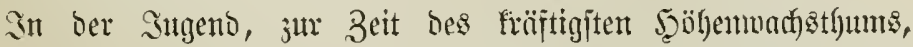

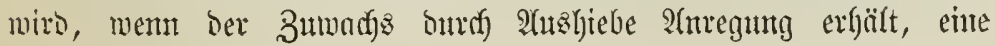




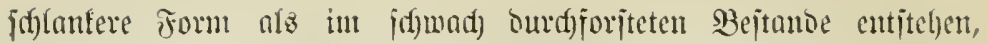

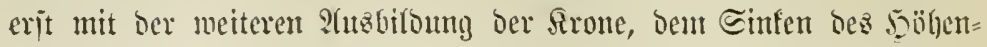
mudjes unt bes laufenten Bumadjes ïberthaut mito in ter 5aupt=

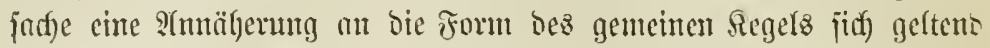

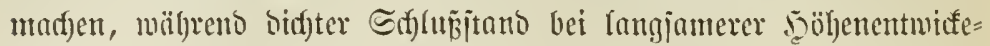

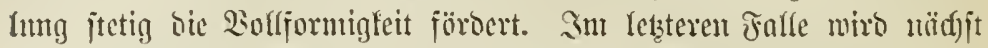

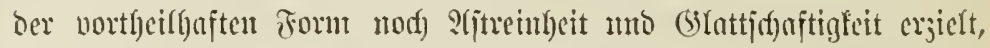

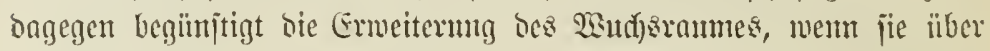

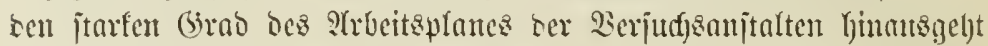

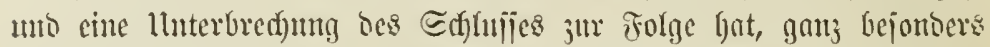

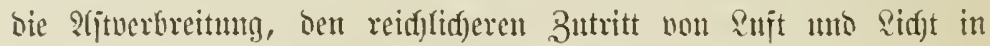

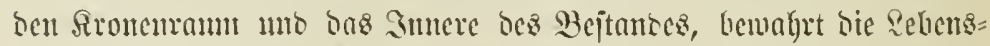

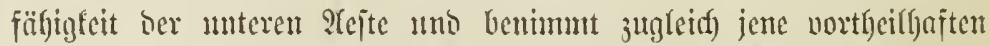
llmjtänoe, meldf)e bas rajd)e s(bjaulen ber bïrren Sejte begünjtigen.

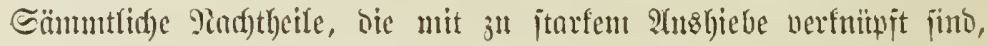

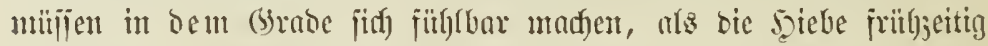

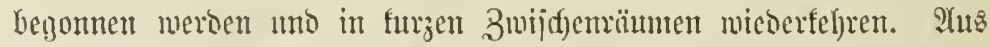

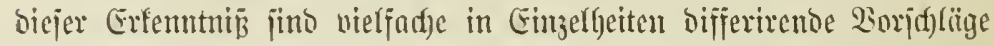

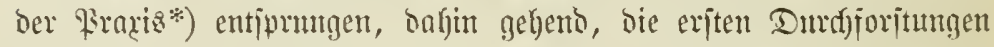

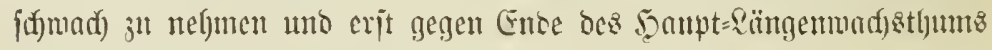
ber Bejtünto jtarf einzugreifen.

\section{c. Gejanmmtntajie.}

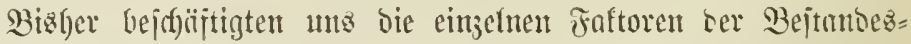

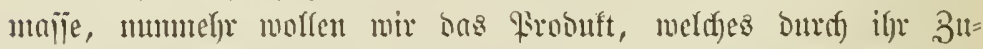

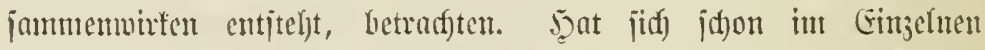

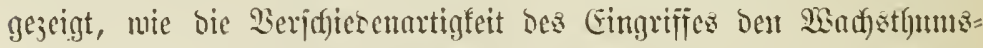

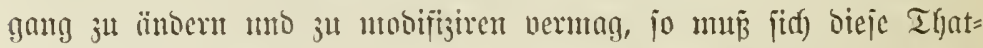

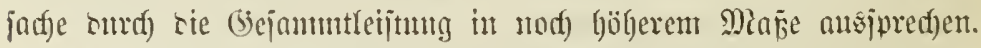

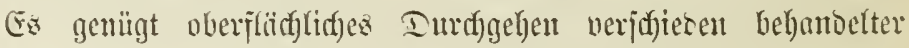

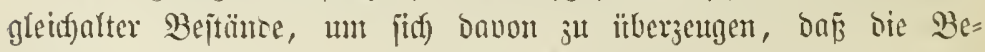

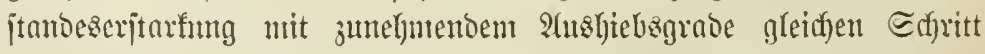

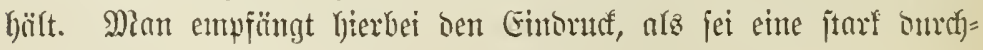

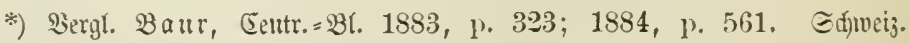
3eitidit. 1885, p. 2\%. 


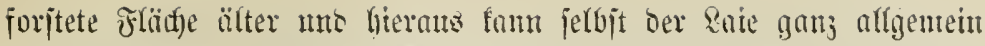

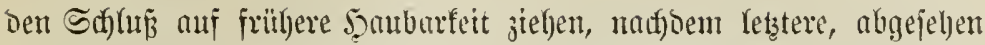

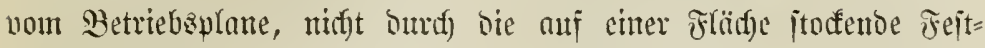
meternuantitït, jonoern burnd bie Stärfebimenfion ber einjelnen

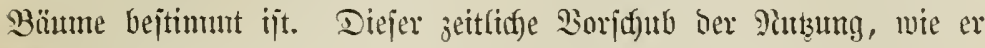

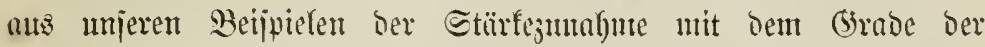
Durdforitung bentlirf) hervorgegt, wiro feites meiteren Bemeifes

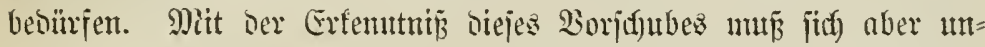

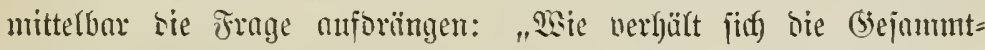

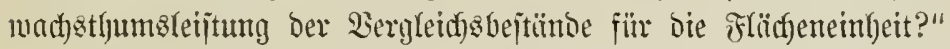

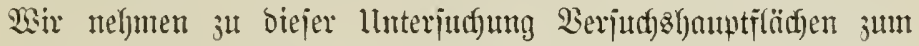

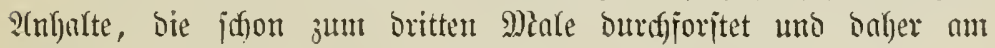

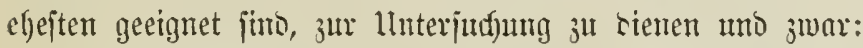

1. einen 51jührigen Fifftenbejtuns, entitanben ourd) 9iatur= veriüntyung in figl. Foritante Sttobeuren, Dijtr. Eieben= thannermulb, s(bth). Solditattfopi.

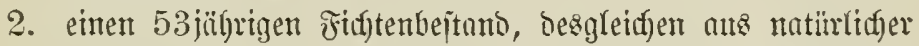
Serjïntgung herworgegangen in figl. Forjtreviere (stajrath), Dijtr. Nieringermats.

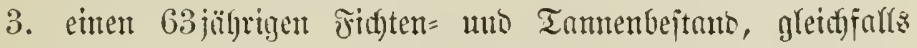
Satmorjiingung, int fgl. Foritumte Dttobeuren, Diftr. Siebentfjamermals; : s(bth). Edjodjenteidf.

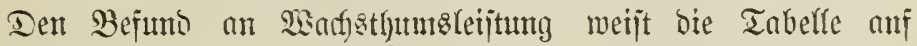
Seite 32 nadf.

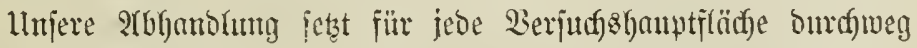
gleiche Etanborts faftoren vorans, jo baj bie Beridfiesentheit ber er=

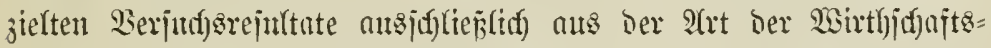
mapregel no ifyer banbljabung entnommen merten fanu umo bes:

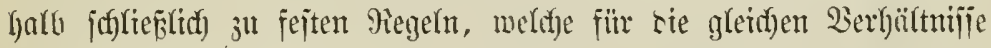

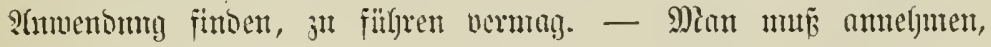
oñ bei gleicher Stanourtsginte glet(f)alterige Bejtünte von terjerben

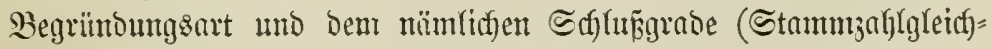

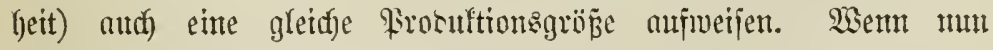

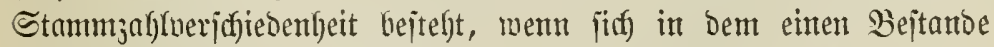

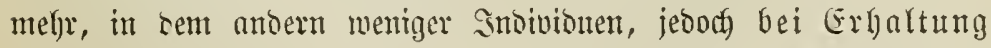

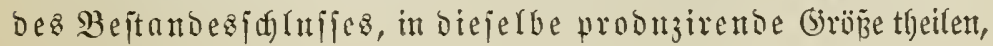




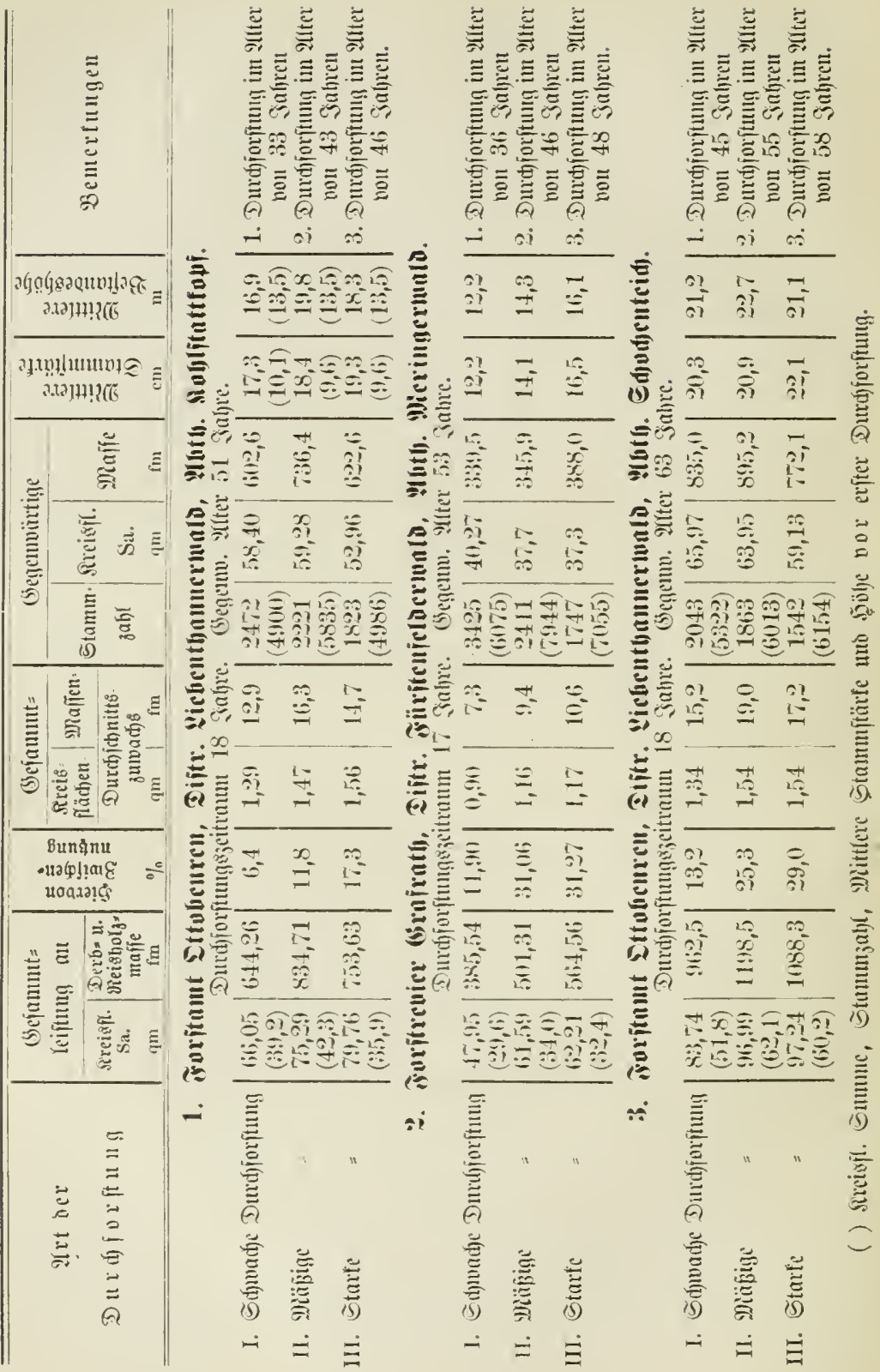




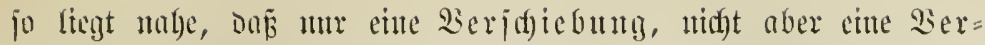

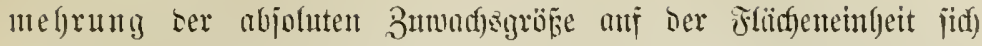

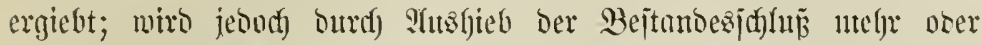

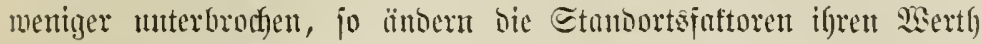

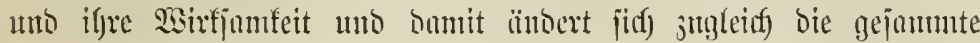

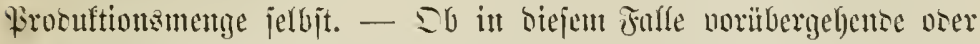
antgaltense Steigerntng erfolgt, funt worlänfig nid)t beniejen merben.

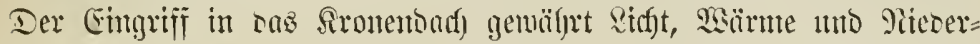
j(d)lägen leidfteres Eintoringen in bas Bejtantosantmere, als bies bein volfen Bejtambesfolujie ber Fall mar; bie cambiale Thätigfeit ex=

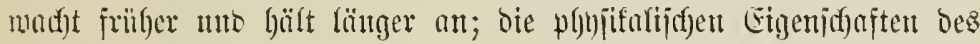

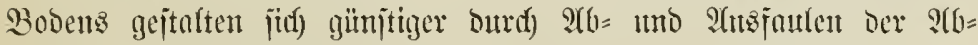

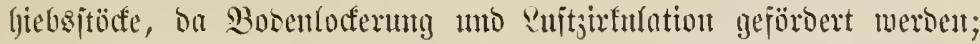

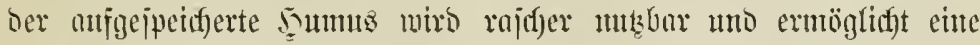

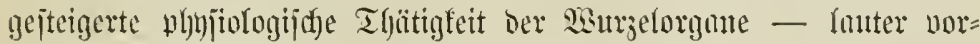

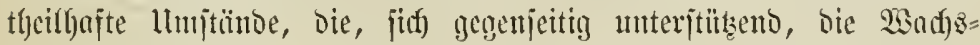

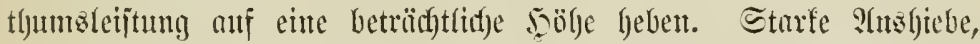

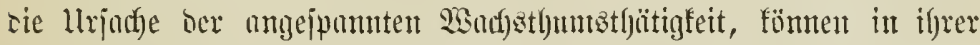

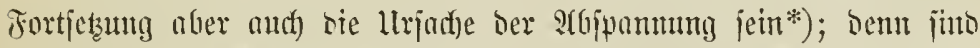

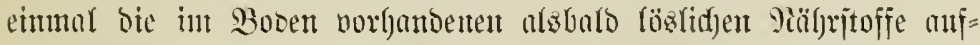

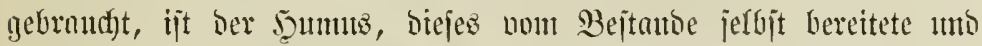
jtänoig ernente Iinngungsmiter, vermbeitet und ijt bie Beptandes= nerjajung nidyt meljr in ber Rage, bie Beoingungen fïr bejien vor=

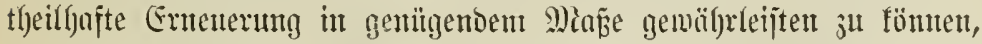

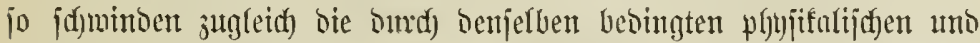

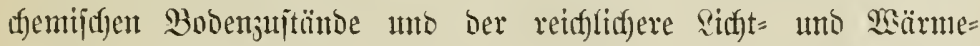

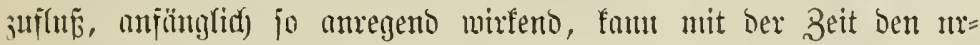

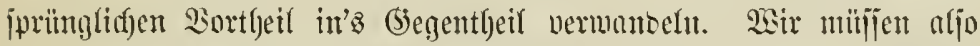

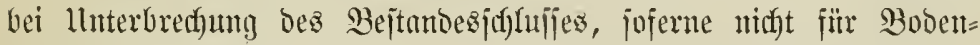

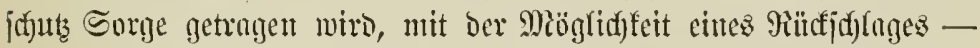

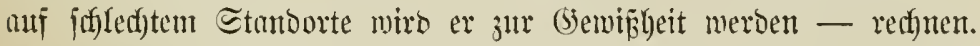

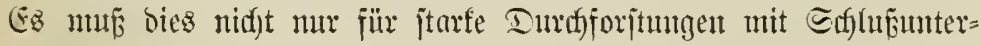
brechung, jontern auff, uno jun in böljerem Manjitabe, fïr bie juäter ju befanoelnoen Siffungen gelteno gemafft meroen.

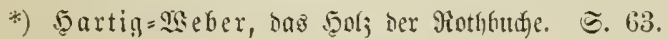




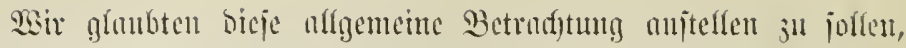

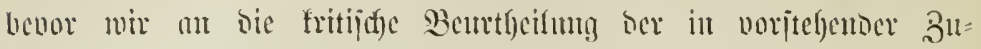

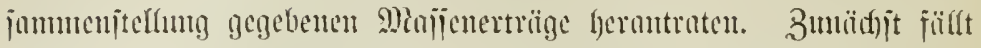

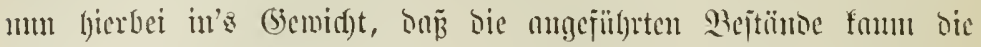

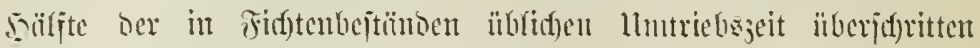

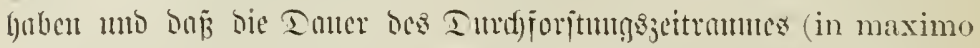

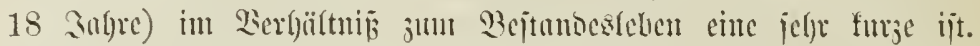

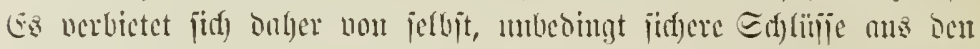

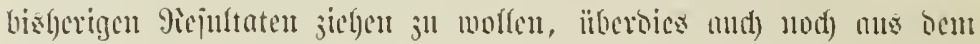

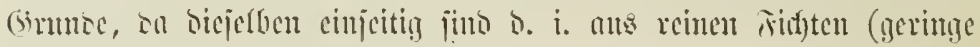

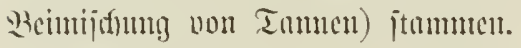

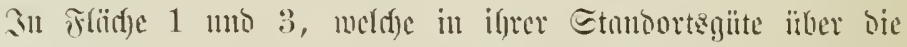

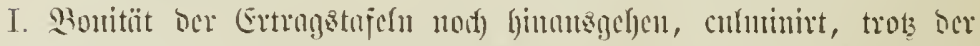

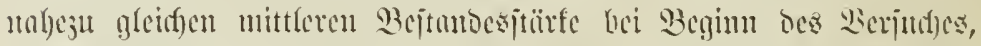

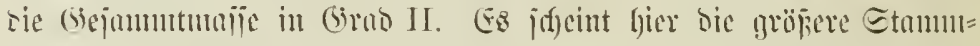

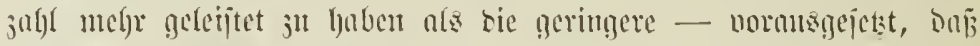

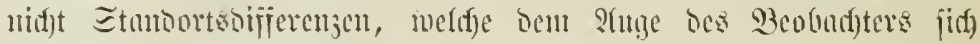
cutjiclyen, sie ltrjutge jims.

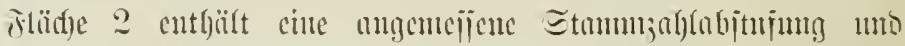

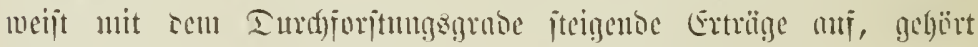
jeboff) ciner geringeren Bonitït un us bie vorigen.

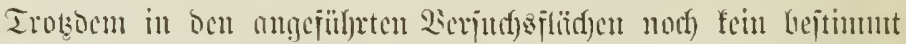

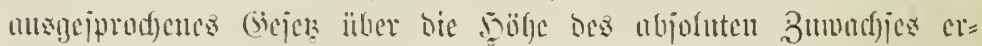

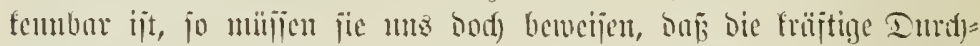

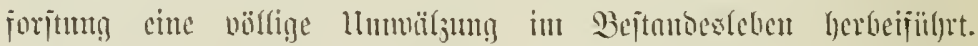

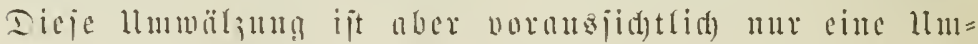

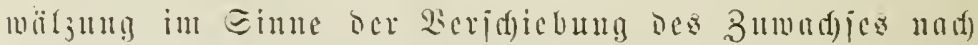

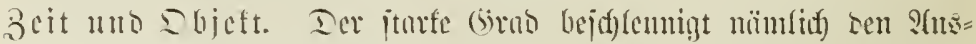

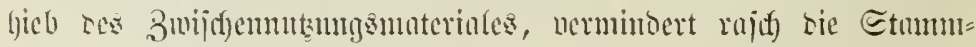

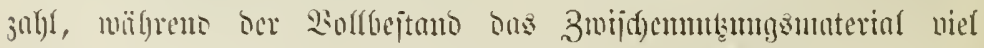

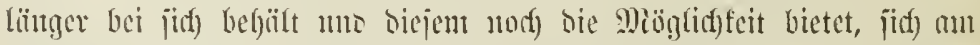

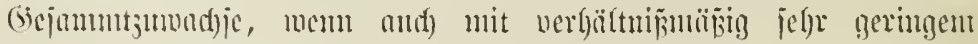
Hitrojente, jull betheifigen.

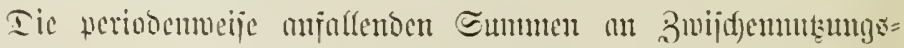

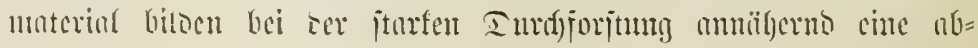




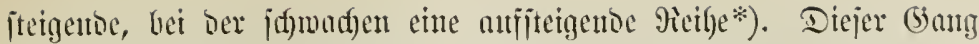

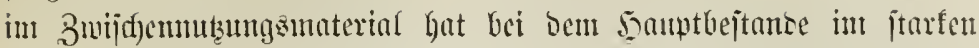

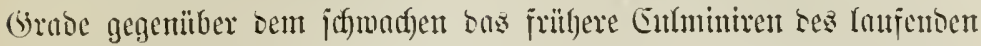

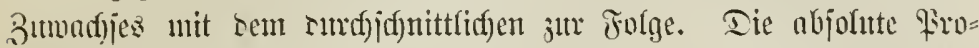

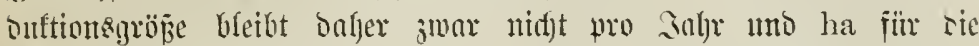

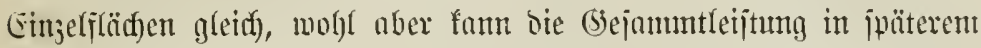

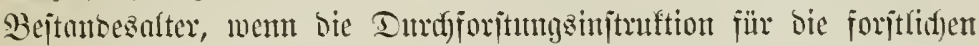
Sierjudgan

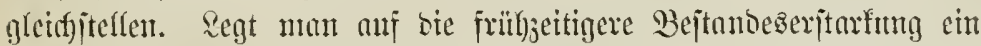
jt gropes (bemicfte (Wangener), jo faun jogar ein Einfen gegent= ither bent Doufbejtance eintreten.

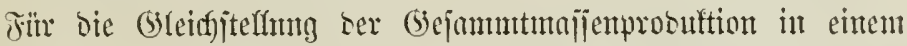
gewijien 3eitpunfte binfte melgr als ein $\mathfrak{2}(\mathfrak{n}$ )ultapunft gegeben jein.

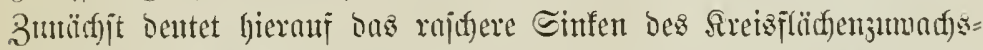

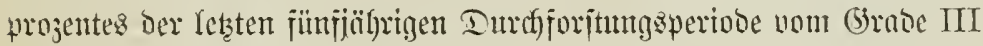

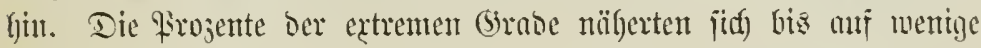
3elnter.

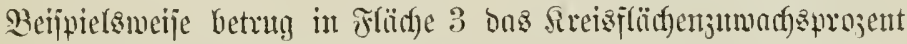

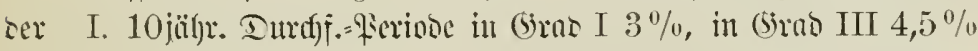
III. $5=\Rightarrow==$ I $2 \%,==$ III $2,1 \%$

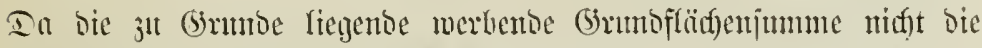

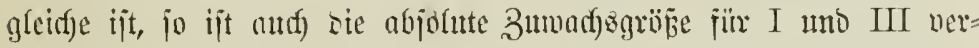

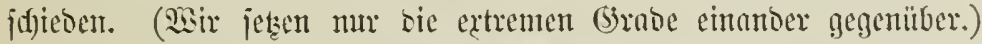

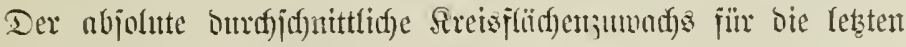

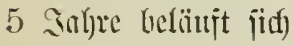

$$
\begin{aligned}
& \text { in I anI } 1,34 \mathrm{~cm} \\
& =\text { III }=1,2,2=
\end{aligned}
$$

Ia num bie mittlere Bejtanbesflübe in beiben Flärfen riejelbe ijt,

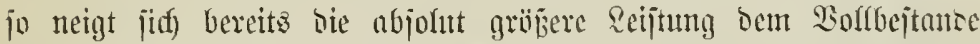

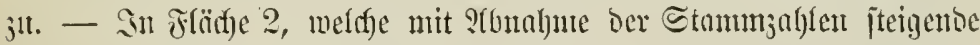
Miajenprosuftion jeigt uno bant mbeoingt in siejer Sinjirfot fïr

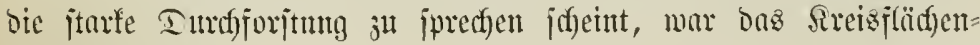
jum(ad) sprozent

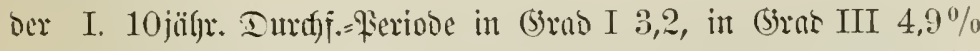
$=$ II. $5=\Rightarrow=\quad=\quad$ I $2,3,==$ III $2,7 \%$

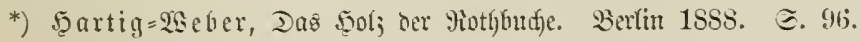




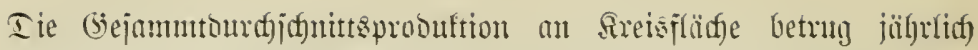
pro ha in Brad I 0,90 qm, in (jirto III $1,17 \mathrm{qm}$,

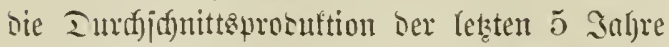

$$
\text { in (jrab I } 0,93 \mathrm{qm} \text {, in Grab III } 1,12 \mathrm{gm} \text {. }
$$

(E) nolfjieht jitf) aljo aud) ljer (auj ber geringerent Bonität) jdjon

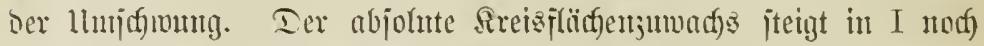

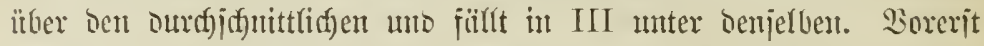

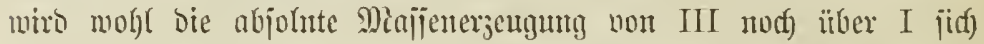

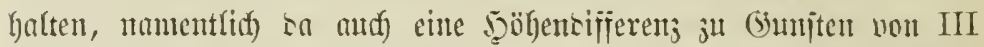

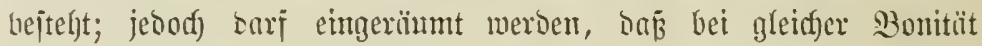

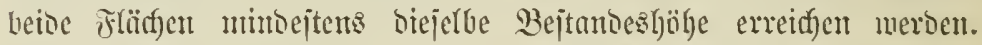

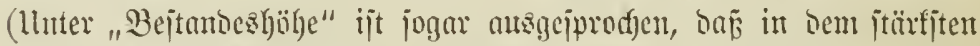
(Grabe cin Einfen unter I jtattfimoen fanm.) Dat jidf mu ber

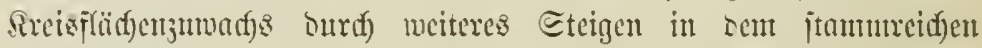
Beitambe Dund) Sinfen in Dem itmmuarmen gleidfgejtelft, jo binrgt

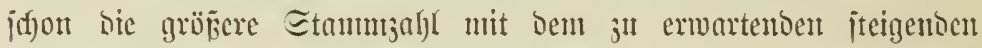

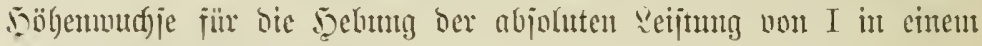
geniijen 3citpuntte ither III.

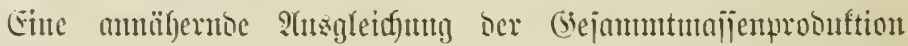
iit jount jiemlidf) walgriffeinfirfy.

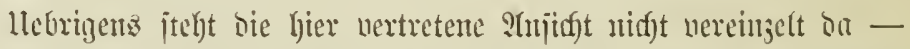
bie von anorer Ecite angejtelften 2 ergleiffungen gaben jofon poitive Ficjultate. So madjte Sammerrath Dorn in Bramidguein, mo= jelbjt man surdf) Beredftigungen gejunutgen war, nod) über ben

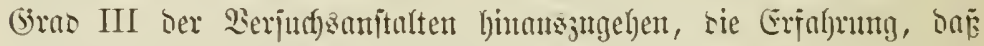
jtarfe Iurdfjorjuntyen in Budfenbejtünoen, jojeme siejclbent jiff) auj jüntgere Bejtüntoe eritrecftent, in bent gemölynlidfen Turnuts in gleiffer Etärfe nifft micberflgolt meroen fonten; man muspte entweder bie

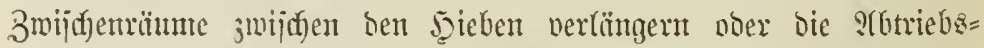
maije ermü̈̄igen. Die (jejammtmajienerträge nad) ben bis= Gerigen Erfagrungen, namentlif auf Bosen mittleres

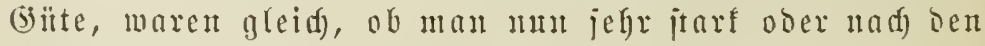
gewölntiden Megeln burdforitete.*)

verner nersanfen mir Exmappad) einte nergletdjente llnter=

*) Iandelm., 3. 1885, ธ. 125 . 


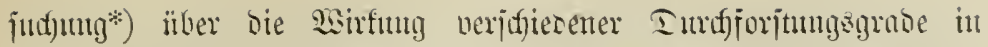

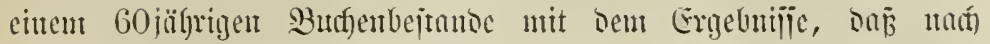

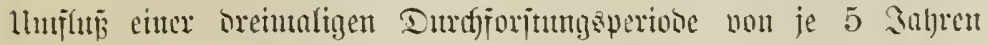

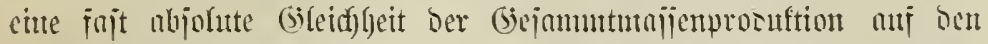

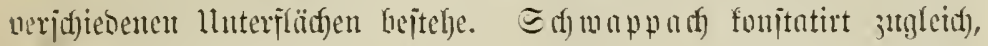

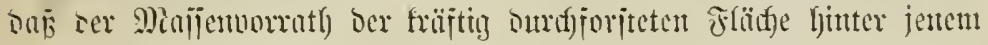
Des Grobes I juridefofieb.

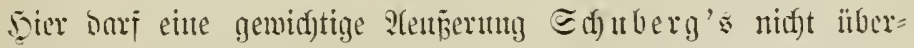

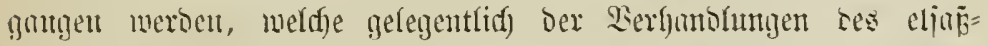

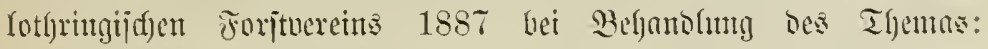

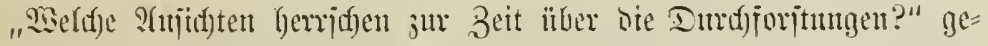
jaflen ijt.**) Diejelbe bejeidfnet sie Mieinutg, man föme surdf

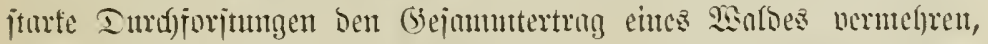

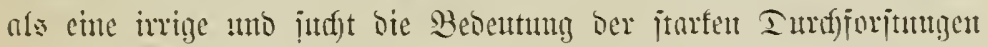
nur in bem erfjöbten sertfertrange. Dieje Eriafjung ijt

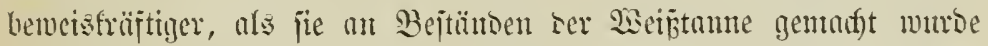

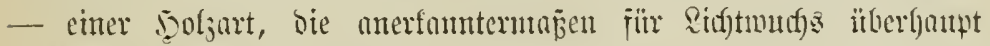
bunfburer ift, als bic fitchte.

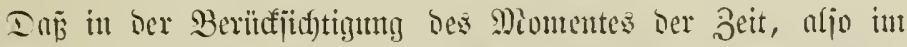

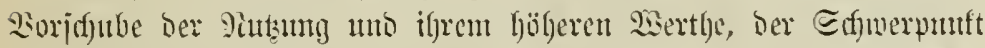

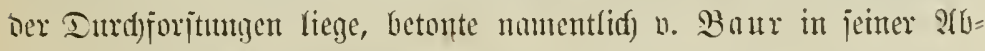

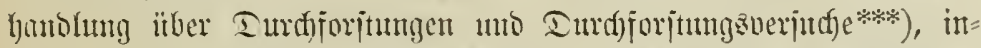

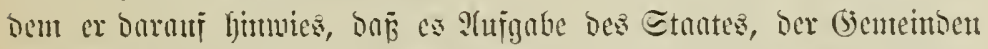
uno ber Privaten jei, Den probuftionsiäljigen Booen jo ju lewirtl)=

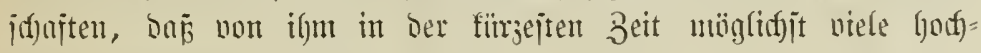
mertfige Frobutte gewomen merbent.

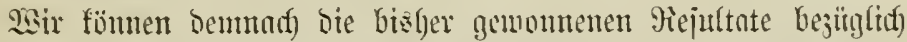

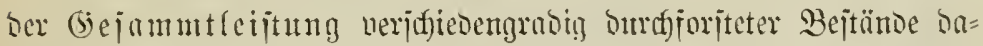

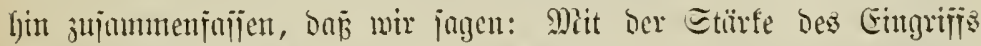

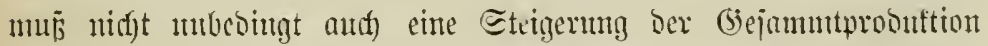

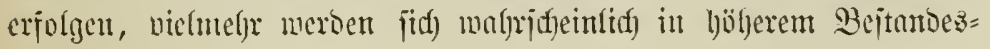

*) Daudm. 3. ․ ร. น. 3. 1885, ๔. 609.

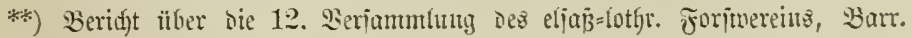
185\%, Є. 23.

\%) (5)anghoier, 28. II. 1884, こ. 209. 


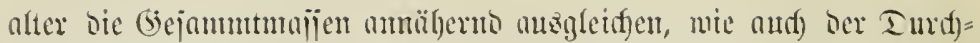
jorjungsantad gemejen jein mag; a

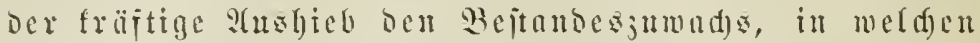

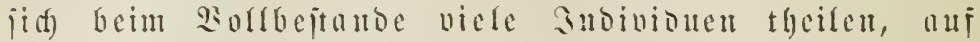
meniger Etämme fonjentrirt, bas onger bie Mägliffeit ber jriberen dingng uno nuter llmpänen höhere (jejammt= mertlerjengung ju ermarten ijt.

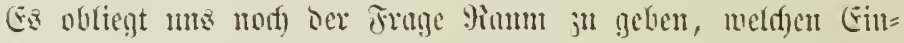

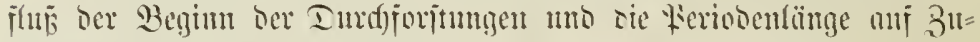

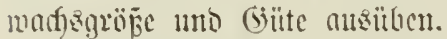

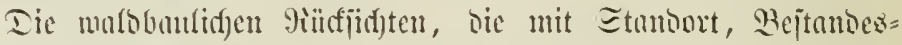

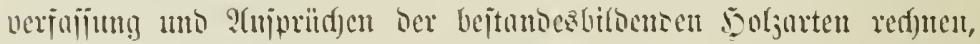

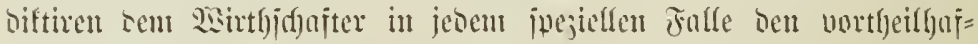
tejten 3eitpunft bes Beginnes. (Es nerbietet namentfidg bie Finjorge

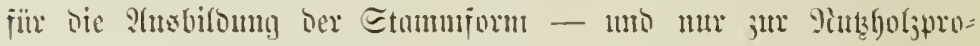

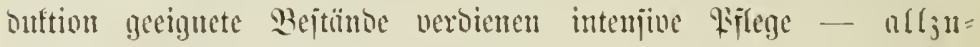

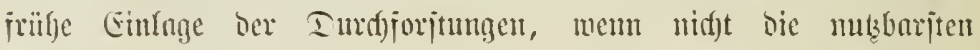

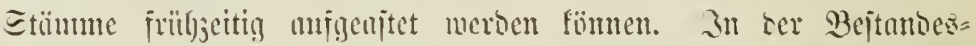

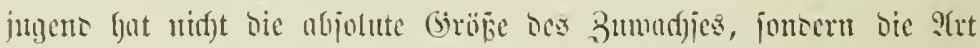
Der ?tnfagerung bas leitente Mintu fïr bie Majuegel res Eingrifies

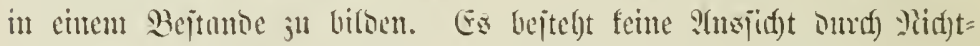

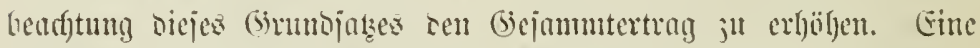

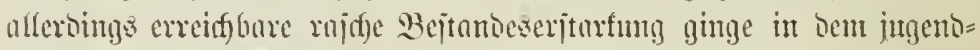

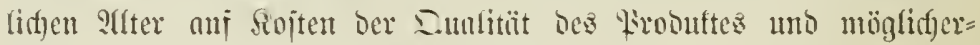

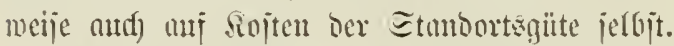

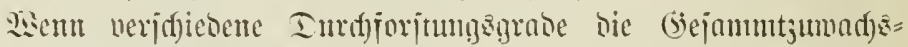
grö̈̈e nidft änorn fömten, jo meroen aud) bie Durdjorjtungsinter=

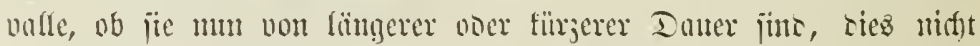

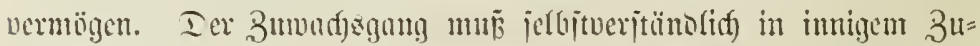
janmenfange mit ber Feriosensuer jtehen. Surje 3mijh)mäume jteigern ben Ejjeft jebes einjelnen (braces in jeiner seije, lange falfen bemielben meniger gervortreten. Eritere bejdleunigen ben Ein=

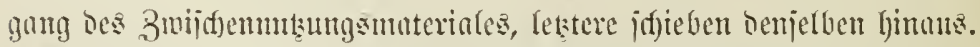

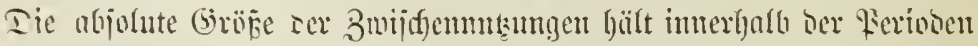
Den umgefefrten (jang cin; jie wiro surd) büufige siseberfebr bes Tiebes verminocrt - Der Zumad) oen bominirenten @tümmen ju= 


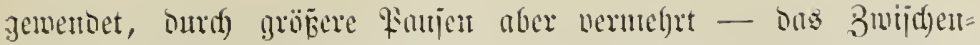

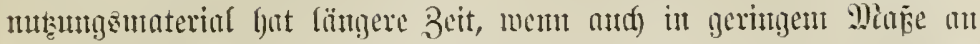

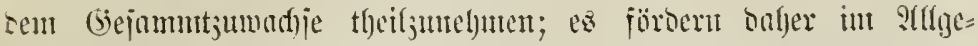

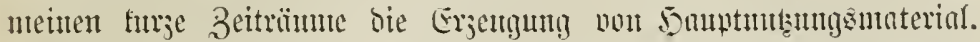

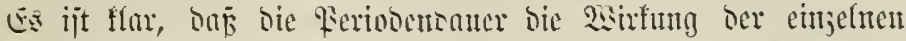

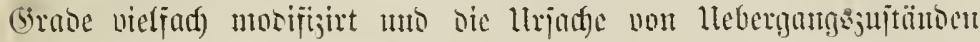

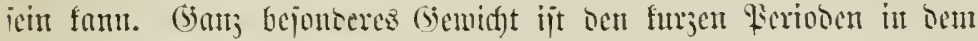
jd)wad)en un mäpigen Gruse heijulegen, meil siejelben bas Mittef

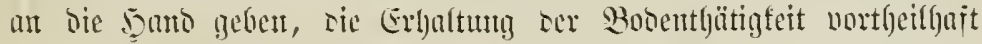

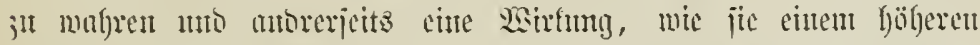
(5rabe afferbings in fïrjerer Beit cutipridft, Gerworjubringen. Iieje

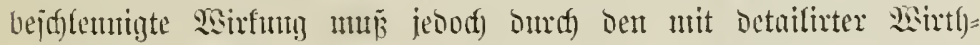

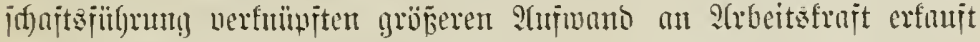

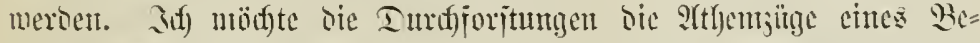

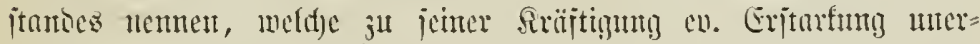

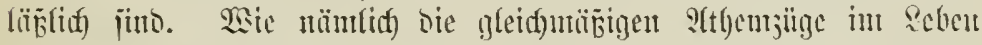

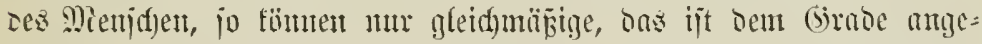

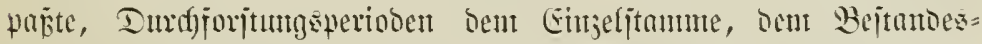

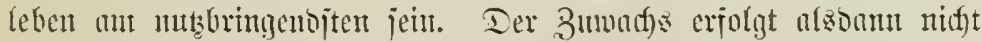

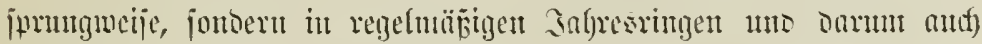
qualitutiv - jomeit bie Etruftur bes Dofjes in Betrad)t fonm in bejter Forin.

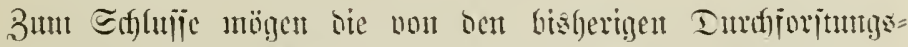

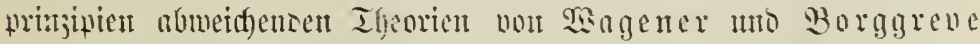

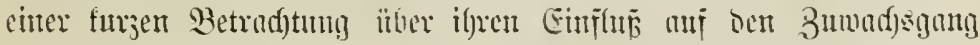

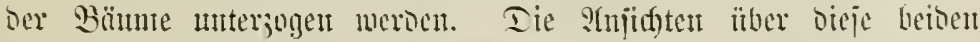

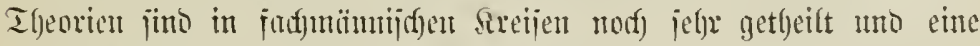
groge Folentif lont jid) an biejelben gefnilipit.

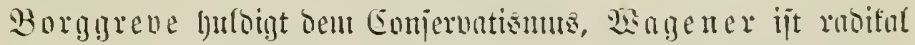
- Baz ijt mit mestigen

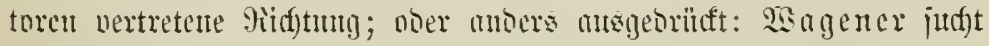
mit jeiner Theorie bie Wrocuftionszeit in ser benfour mögfidjten

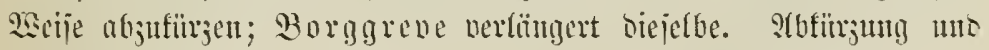

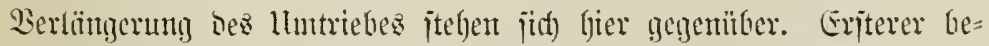

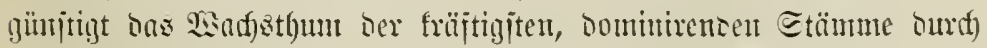

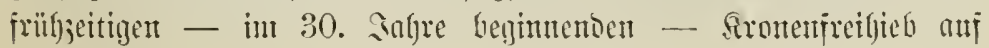




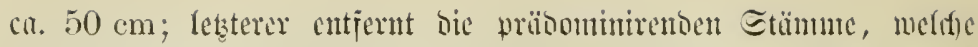

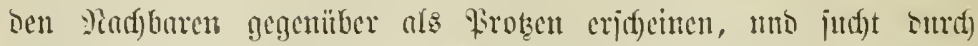

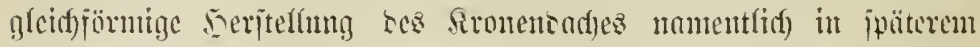

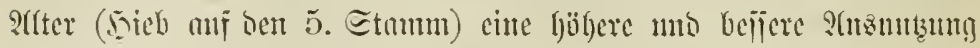

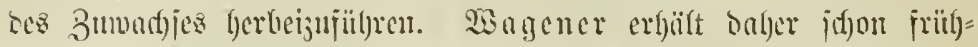

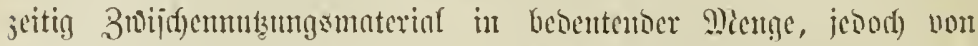

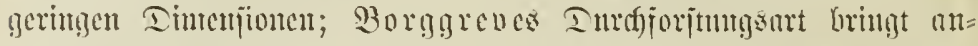

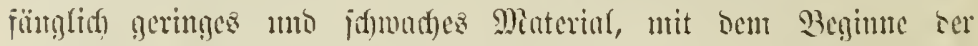

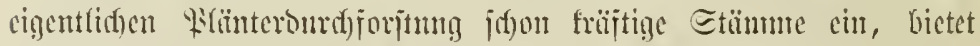

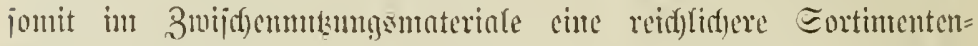

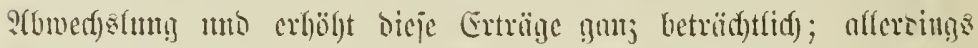

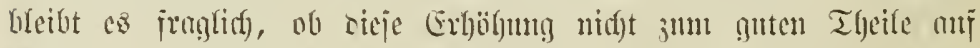

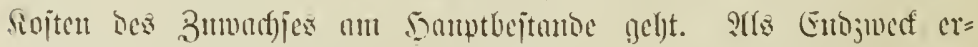

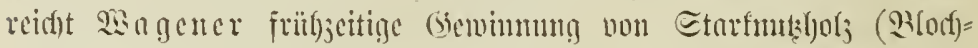

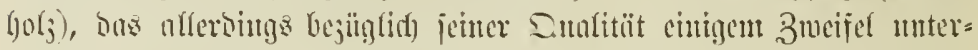

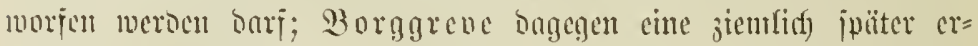

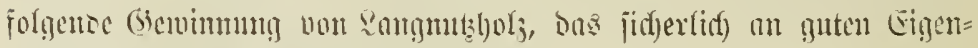

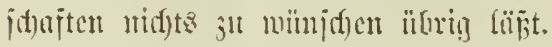

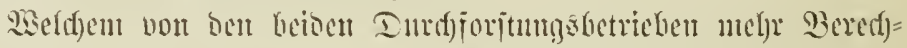

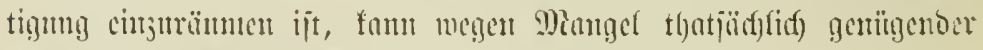

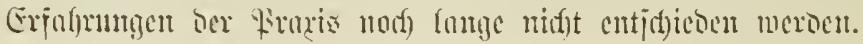

\section{Micntalitität.}

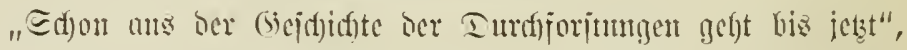

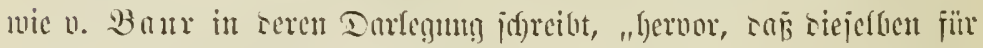

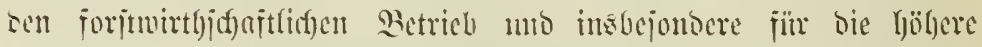

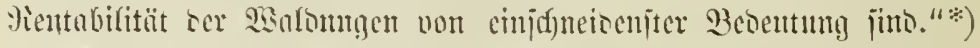

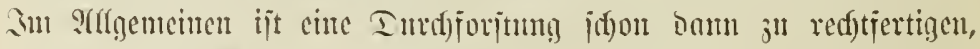

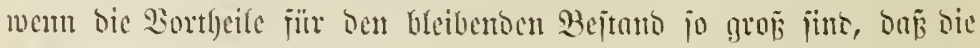

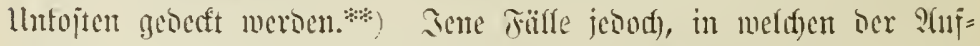

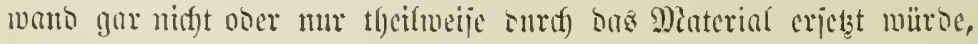

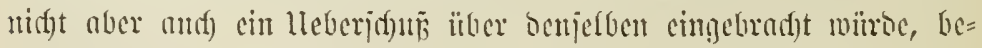

*) Baur Eentrbl. 188\%, ๔. 21.

*) Eiterr. \$siertelj. 1854, E. 15. ๒. (5 uttenberg, Etutitif bez Durd)= forîtungăhetrieges. 


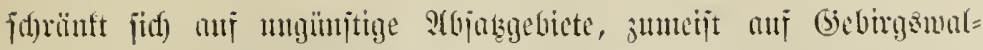

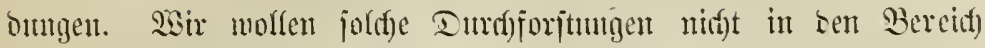

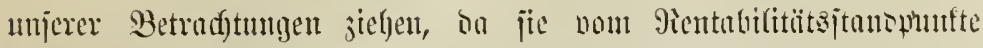

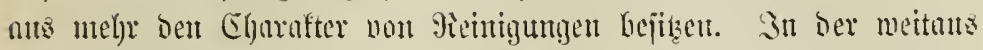

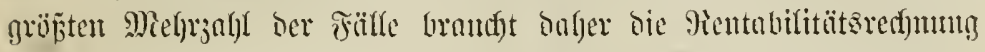

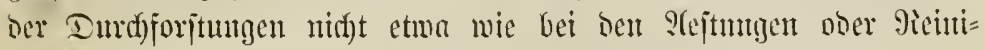

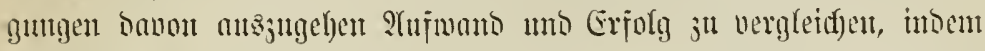

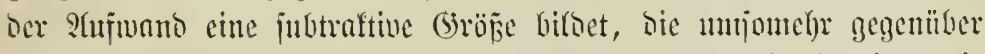

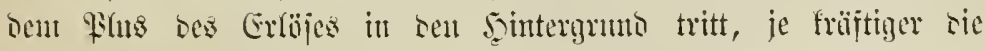

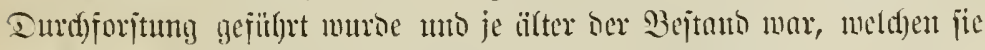

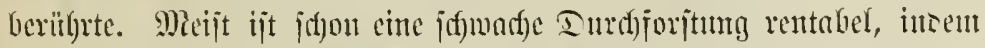

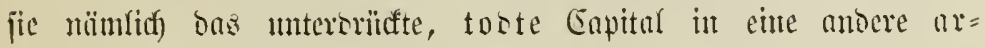

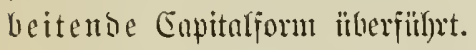

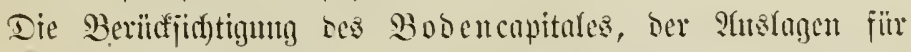

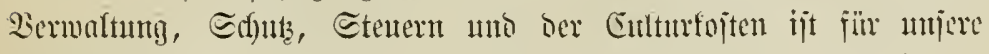

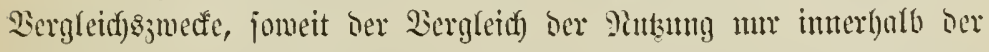

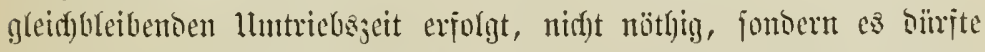

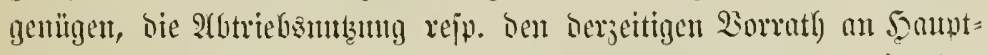

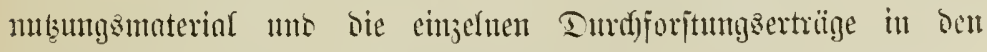

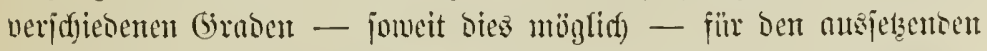
mie aud) fürr ben madghaltigen Betrieb jur Bentyeilung ber gien=

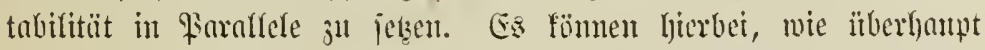

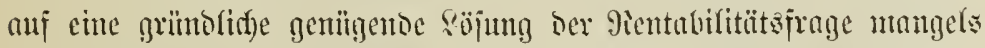
bes grunblegenoen Miaterinles uerjidftet meroen nus, Folgerutgen,

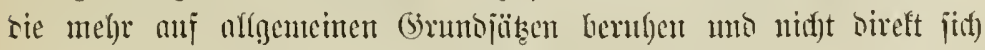

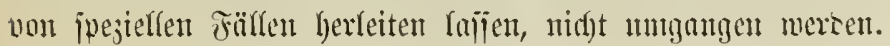

\section{a. 2lus-jebender Betrieb.}

Es ijt erflürlidf), menn ein Bejtante einen möglidyjt hohen Evlös ju erringen trachtet mo jul

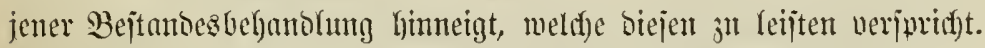
Gejetżt mut, man vergleich)e von biejem Stamopunfte aus bie er=

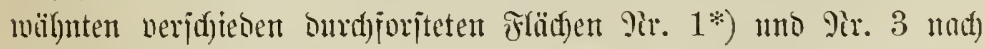

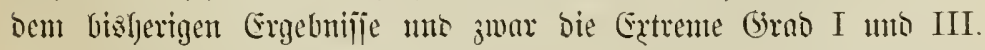

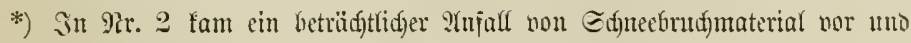

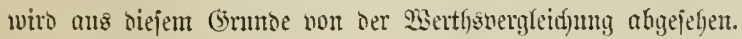


Sln̈de Jir. 1, :(oth. Moflitattfupi.

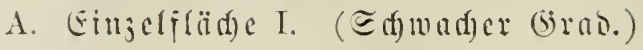

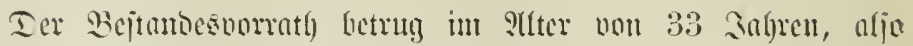

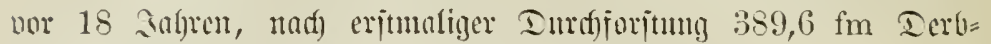

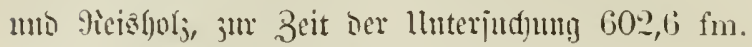

I ie Imdjoritumgertrüge marien ab

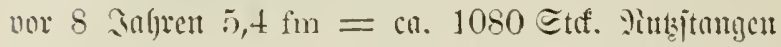

(unittlere Etürfe $4 \mathrm{~cm}$, mittlere vänge

\% m) pro 100 Etff. 2 Mif. . . . = 21,6 Mit.

$=5=20,8 \mathrm{fm}=$ ca. 1300 ङtuf. Yinßzitunger

(mittlere 巨türfi T $\mathrm{cm}$, mittlere vängze

$8 \mathrm{~m})$ pro 100 Ettf. F Mit. . . . $=91,0=$

$=0=15, \pm \mathrm{fm}=$ ct. $: 3 \pm 0$ Etcf. Iinzitungen

(unittlere 巨tärfe $10,5 \mathrm{~cm}$, mittlere vämige

$11 \mathrm{~m})$ mu 100 Etct. 18 iff. . . . = $=61,:=$

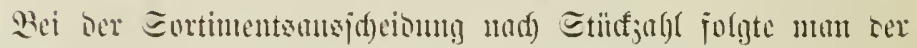

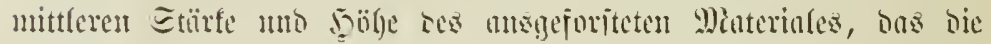

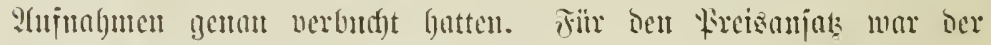

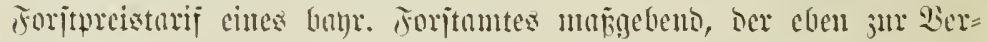

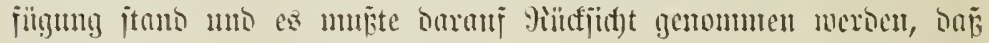

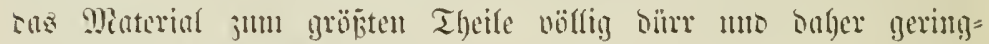
merthiger als grïnes mar.

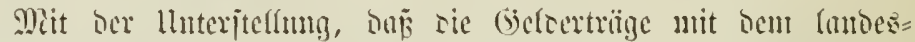

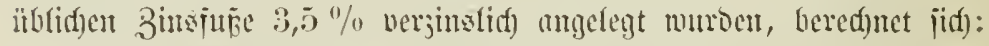

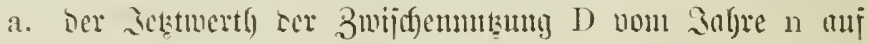

$$
\begin{aligned}
& D_{\mathrm{s}}=21,6 \times 1,035^{\mathrm{s}}=28,5 \mathrm{2} \mathrm{if} \text {. } \\
& D_{i}=91,0 \times 1,035^{5}=108,3= \\
& \mathrm{D}_{0}=\cdot \cdot \cdot \cdot \frac{101,2=}{\Xi_{\mathfrak{n} .} 198,0 \text { Mif. }}
\end{aligned}
$$

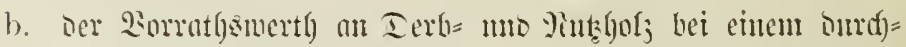

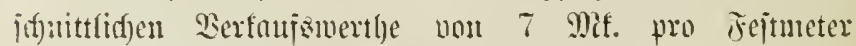

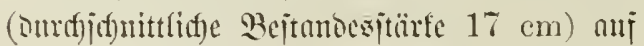

$$
602,6 \text { a } \div \text { Mif. }=4218,2 \text { Mif. }
$$

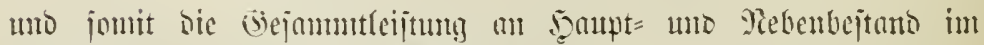

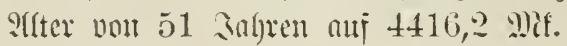




\section{B. Ėujelilüd)e III. (ভtnrfer Grar.)}

Ier Bejtunoesnorratl) betrug im צfter von 33 Sofflen math

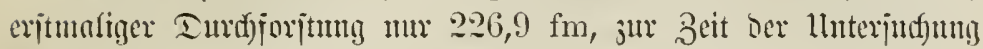
$622,6 \mathrm{fm}$.

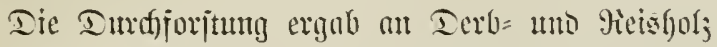

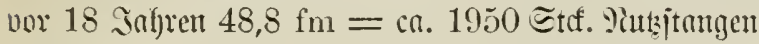

(8 cm mittlere Etärfe uno $9,5 \mathrm{~m}$ mittfere Qünge) pro 100 ङtof. 12 Mff. $=234,0$ Mif.

$=8=31,0 \mathrm{fm}=$ cı. 960 @tff. Pinţītungen

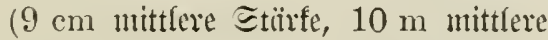

Pïnge) pro 100 @tff. 20 Mif. . . = 192,0 $=$

$5=38,9 \mathrm{fm}=\mathrm{ca} .650$ Etcf. Piuţitangen

(11 $\mathrm{cm}$ mittfere Stïrfe, $13 \mathrm{~m}$ mittfere

Qünge) pro 100 @ttf. 25 Mif. . . = $162,5=$

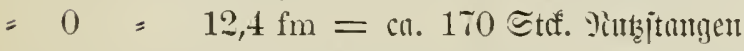

wro 100 ङtcf. 30 Mif. . . . . $=51,0=$

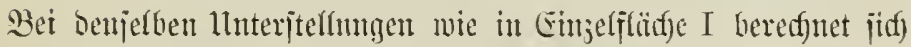

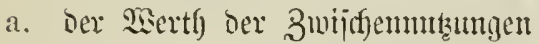

$$
\begin{aligned}
& \mathrm{D}_{18}=234,0 \times 1,035^{1 \mathrm{*}}=4329 \text { 920 } \mathrm{it} \text {. } \\
& \mathrm{D}_{\mathrm{s}}=192,0 \times 1,035^{\mathrm{s}}=253,4= \\
& \mathrm{D}_{5}=162,5 \times 1,035^{5}=193,3= \\
& \mathrm{D}_{0}=. . . . \quad .=51,0= \\
& \text { ङ1. 930,6 Mit. }
\end{aligned}
$$

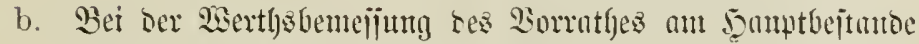

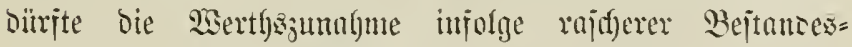
exjturfunty (mittfere Etärfe $19 \mathrm{~cm}$ ) in Yedfunng ju nelgmen jein uno onber ber Freis pro jejtmeter mi 8 gif. jidf itelfen.

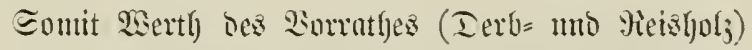

$$
622,6 \mathrm{fm} \times 8 \text { Mif. }=4980,8 \text { Mif. }
$$

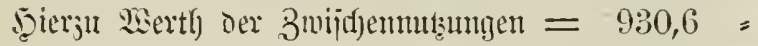

$$
\overline{\Xi_{n} .5911, \pm \mathfrak{M i f}_{\text {. }}}
$$

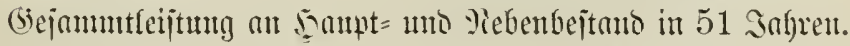
2ergleifft man cent Ertrug von Flätye I mit 4416,2 Mit. unt 


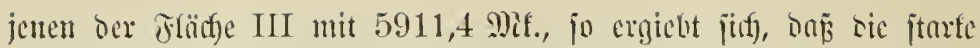

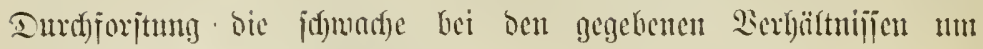
ca. $33 \%$ in Extrage itbertrijit.

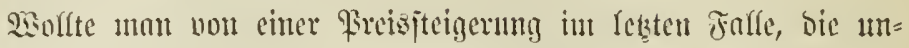

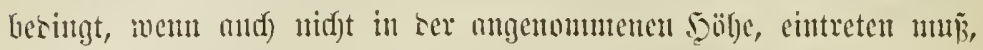

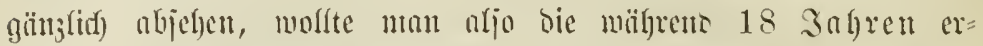

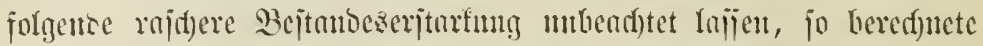

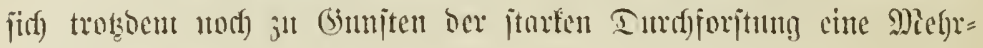
reijtung unn $20 \%$.

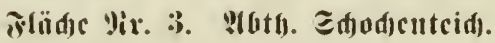

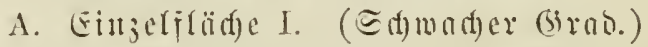

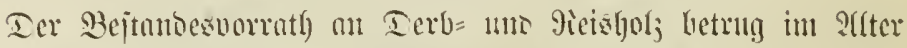

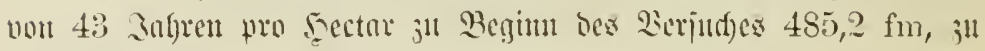
Enoc $835,0 \mathrm{fm}$.

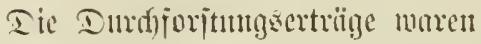
nor 8 3aflure $13,4 \mathrm{fm}=\mathrm{co} .1220$ Etcf. Sinkjtuntgen

$(6 \mathrm{~cm}$ mitttere Etärfe, $7,5 \mathrm{~m}$ mittlere

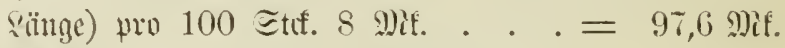

$=5=64,1 \mathrm{fm}=\mathrm{ca} .1600$ こtcf. Pittis 1 tungen

$(9 \mathrm{~cm}$ mittlere Etï̀te, $12,5 \mathrm{~mm}$ mittlere Y'ingc) Mro 100 Etct. 18 Mit. . . . $=288,0=$

$=0=48,0$ fin (ju $3 \mathfrak{r e n t u l g o f}$ mtigentbeitet) Hro Fejtnter 4 Mif. . . . . . . $=192,0=$

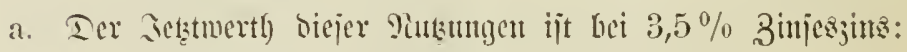

$$
\begin{aligned}
& \mathrm{D}_{\mathrm{s}}=97,6 \times 1,035^{\mathrm{s}}=128,5 \mathrm{Mi \textrm {f }} . \\
& \mathrm{D}_{5}=288,0 \times 1,035^{5}=342,7= \\
& \mathrm{D}_{0}=\cdot \cdot \cdot \cdot \cdot=\frac{192,0=}{663,5 \mathrm{MR} .}
\end{aligned}
$$

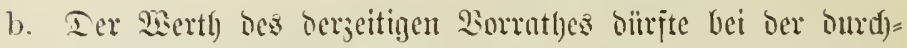

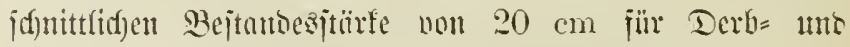

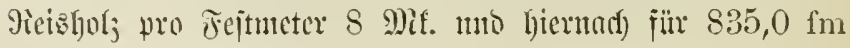
$=6680$ Mif. Ketragen.

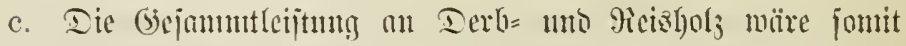
7343,5 Mif. 


\section{B. Einjelfläde III. (Sturfer (jrab.)}

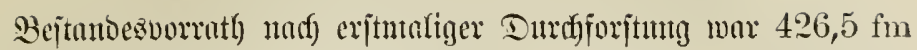

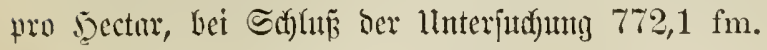

Die Dumbjorjtungen liejerten:

vor 18 3afren $125,5 \mathrm{fm}=$ ca. 2700 Stct. Piutijtnutgent

(10 cm mittlere ভtïrfe, $12,2 \mathrm{~m}$ mittlere

Särne) pro 100 ๔ttf. 20 Mif. . . . = 540 Mif.

$8=146,2 \mathrm{fm}=$ ca. 2000 Stct. Pittżitungent

$(12 \mathrm{~cm}$ mittlere Stïrffe, $14 \mathrm{~m}$ mittlere

Qünge) pro 100 Strf. 25 Mif. . . . $=500=$

$5=22,1 \mathrm{fm}=\mathrm{ca} .220$ Stcf. 2utusitangen

(13 cm mittlere Stürfe, $17,5 \mathrm{~m}$ mittlere

Sïnge) mro 100 Stcf. 35 Mif. . . . = $77=$

$0=22,5 \mathrm{fm}=$ ca. 200 Stcf. Pitujitmingen

(14 cm mittlere Etärfe, $17 \mathrm{~m}$ mittlere

Sünge) pro 100 Stcf́. 40 Mif. . . . $=80=$

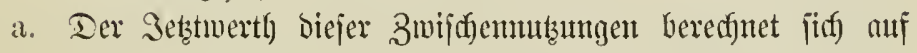

$\mathrm{D}_{18}=540 \times 1,035^{18}=999,0$ Mit.

$\mathrm{D}_{\mathrm{s}}=500 \times 1,035^{8}=660,0=$

$\mathrm{D}_{5}=77 \times 1,035^{5}=91,6=$

$\mathrm{D}_{0}=\cdot \cdot \cdot \cdot \frac{80,0=}{\text { ভa }_{\mathrm{a}} 1830,6 \mathrm{Mif} .}$

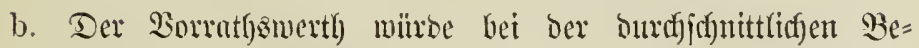
jtantesftürfe von $22 \mathrm{~cm}$ unb eintern Greeije non 9 Mrf. pro Fejtmeter auf

$$
772,1 \times 9=6948,9 \text { Mif. }
$$

jid) jteflen atto

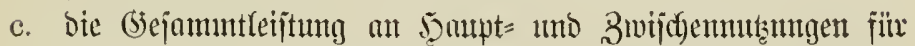

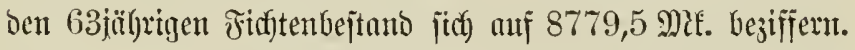

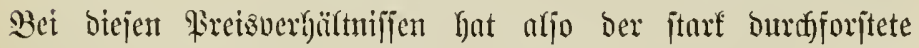

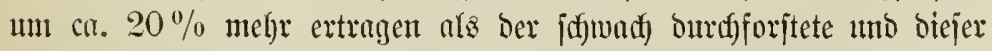
llmitanto ijt, wie bie Biffern in ben beioen angeüüfrten Flärfen jeigen, jun grö̈̆ten Theile ben ju verjfitenenen Zeiten mit verjdjie=

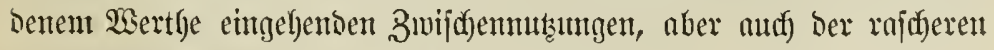
Bejtanbeserftarfung in III. (5rabe ju veroanfen. 


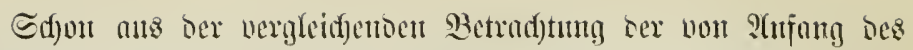

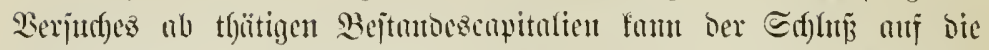

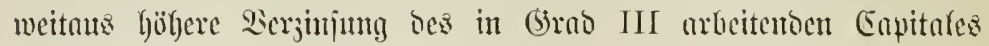
gejogen werour. Eo mar in släctie 1

Grno I hei cinem ?hufungscapitule non $389,6 \mathrm{fm}$

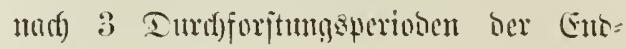
wertl) mur

4416,2 Mif.

III hei cinem stufungscapitale un 206,9 fm

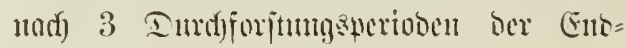
merth nou

creeifft moroen.

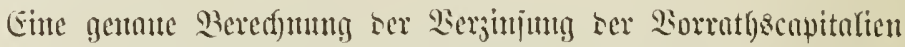

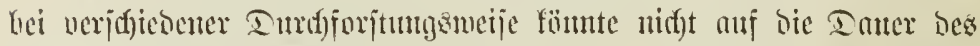

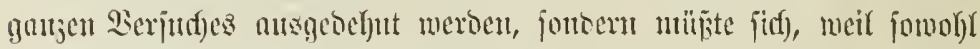

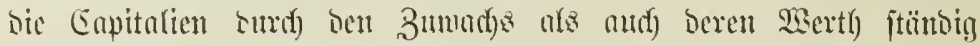

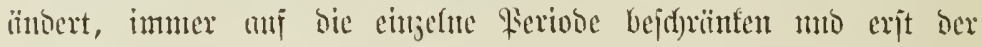

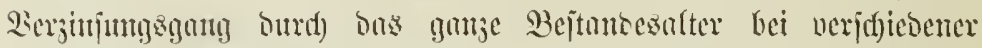

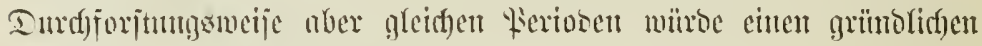
wergleidgenton Einbficf gemälyren.

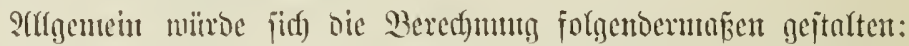

Es jei px bas gefuddete Firnjent, ju meldfen m, ber Sorrnth

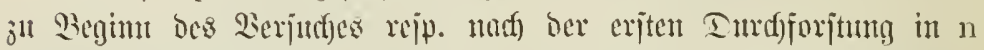

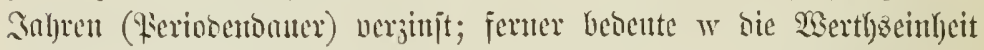

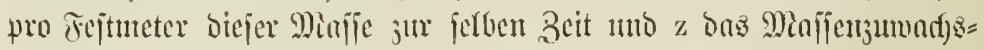

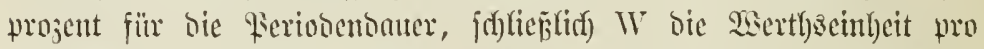

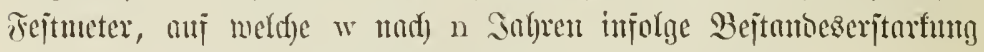

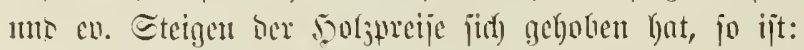

$$
m \cdot w \cdot 1,0 p_{x}^{n}=m W+m\left(1,0 z^{n}-1\right) W
$$

ober bei sercinfadfunty ber (bletd)ung

$$
1,0 \mathrm{p}_{\mathrm{x}}^{\mathrm{n}}=1,0 \mathrm{z}^{\mathrm{n}} \frac{\mathrm{W}^{\circ}}{\mathrm{W}} \text {. }
$$

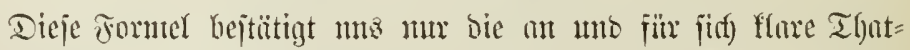

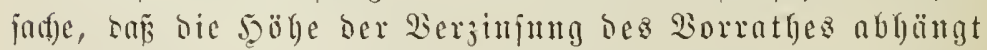

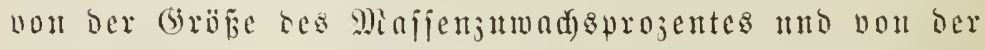

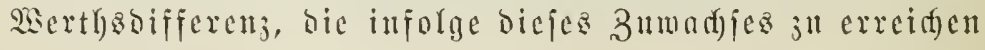




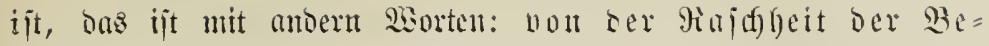
ftanbeseritarfing.

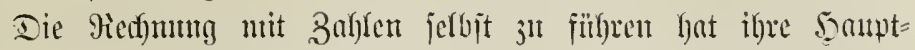

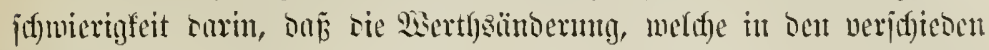

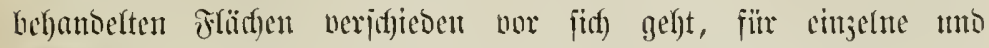

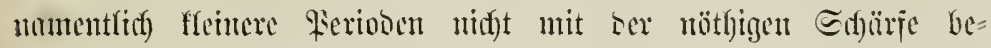

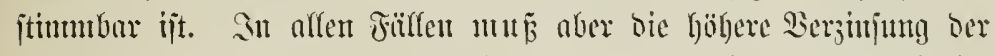

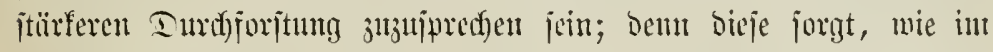

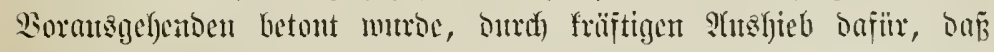

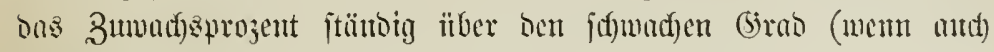

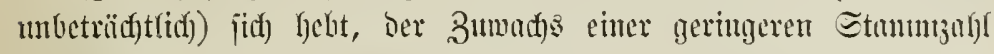

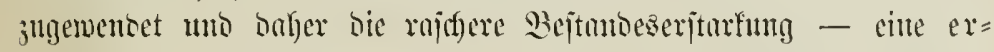

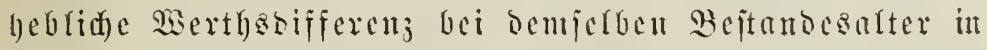
(5rab III gegenitber Grat I - exzielt miro.

senn fiernit bie Etärfebintenjon in crjter Einte als werths:

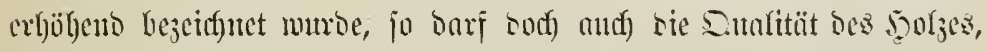

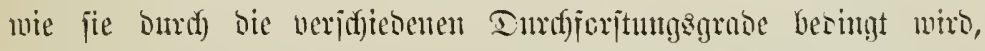

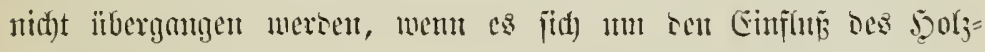

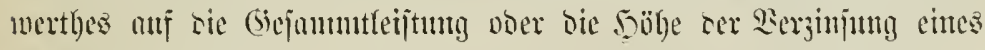
Bejtantos hancelt.

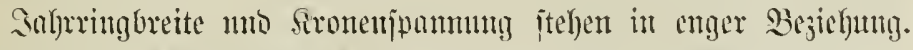

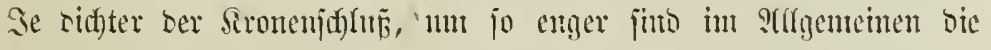

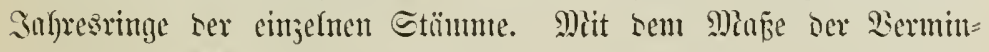

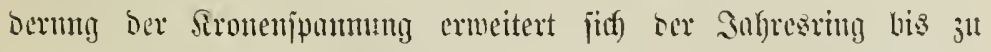

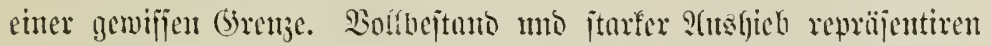

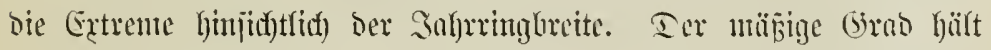

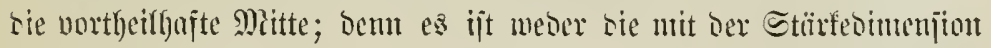

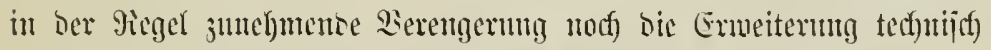

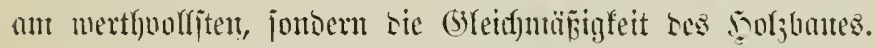

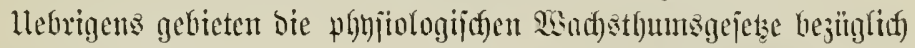

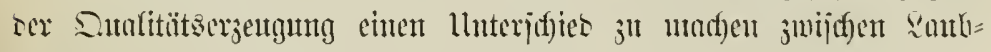

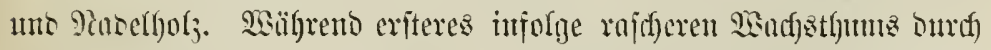

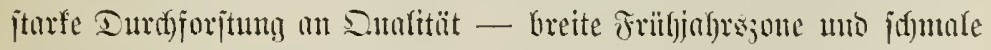
Serbjtjone - gewhunt, nerliert in gleichem Maje sos retżtere. So jeood) biejer Satz hinjidftlid) ber asirfung sar Dur(f)jorjtungen in

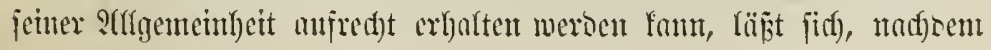




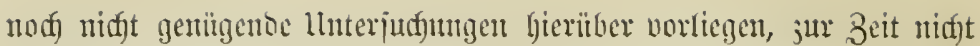
bejtimntt beloumten.

Ebjannt bic ju erjelenten in matontijaen Ban begrümeten

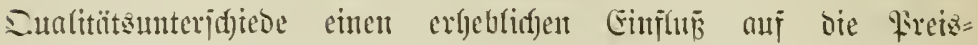
beptimmung babest uno für ober gegen eine Turdjorjtungsmagregel juredfen fömten, jo miro bod) bei ben meijten Sonljnten - menigitens Eqfattholjarten - Der Etc̈rfe= unb Ë̈ngebiutention Der Dantptantheil

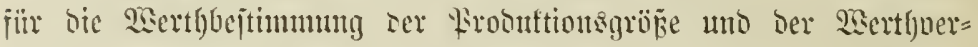
äntorung jufalfen. Sies gilt jelbjtwerjtüntolid) mie für ben fonfretent Bejtanto, jo aud) fiir cinen ganjen Betriehzistomllex.

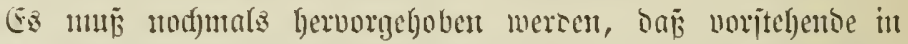
fonfreten Bejtüntoen angejtellte Piecfunng jicf mu nni bie Bejtandes:

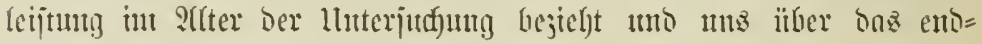

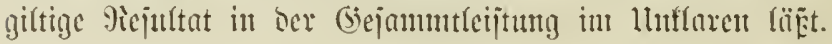

\section{b. 2iadihaltbetricb.}

Es jeien jmet mormale Betriebaffajien anj bemjelben Etamorte

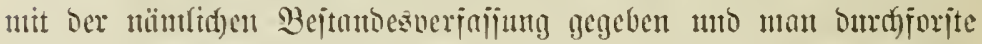

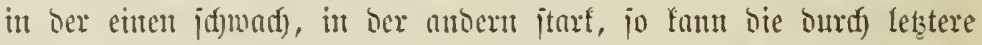

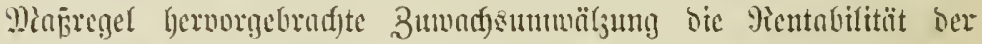

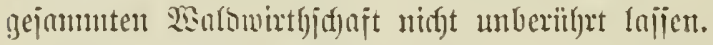

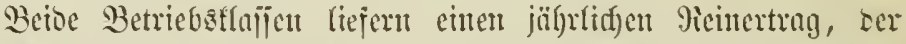

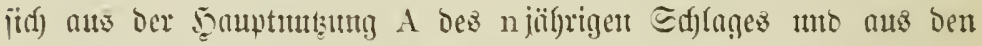

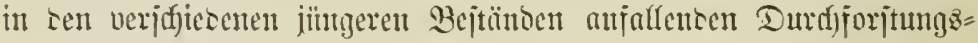
ertrïgen $\mathrm{D}_{\mathrm{a}}+\mathrm{D}_{\mathrm{b}}+\ldots$ jujummenjęst.

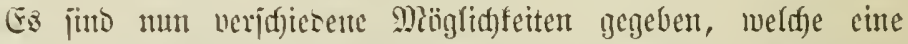

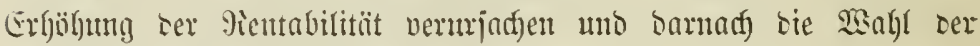
Iurfforjungsant bejtmmen fömen:

1. Bei gleifbleibender llutricbsjeit Steigerung Des albtriebs= ertrages noer ber einjelnen Imdajoritungzergebnijie ober betoer jugleidf);

2. bei gleiffen Reinertrïgen imterlyalb Derjelben Beit Erjparung an Froduftionsfopttal;

3. Erjichung eines gleidlyohen Pieinertrages in fingerer 3eit

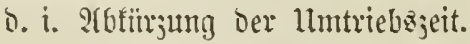

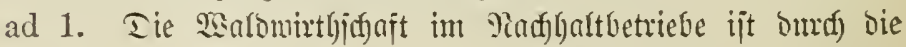




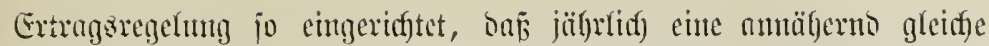

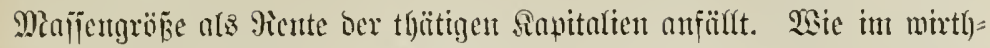

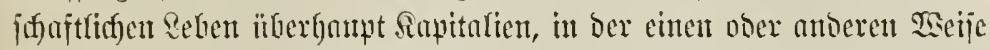

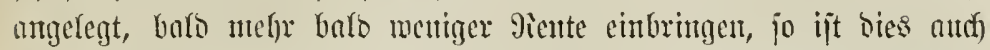

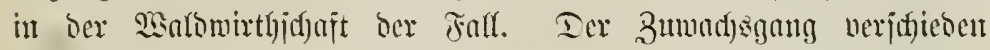

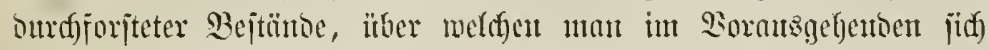

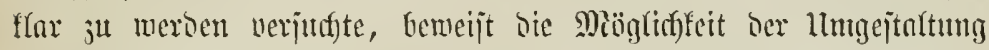

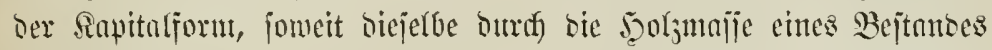
repriajentirt ijt, uno sicje liefert bie ser llmgejtaltung entipredfente

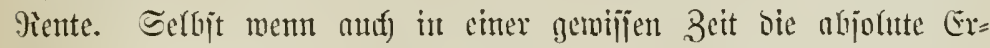

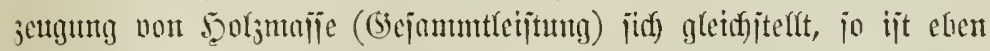
bod) bie frorm, in weldyer jie geboten wiro, einte nubere unt samit

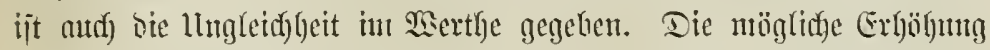

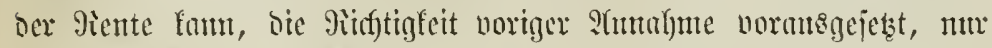

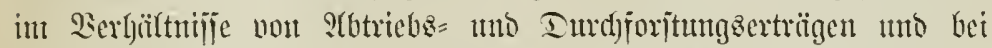

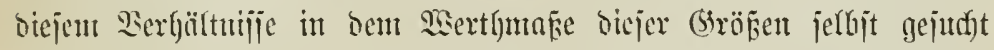

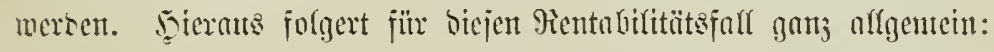

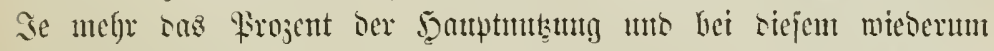

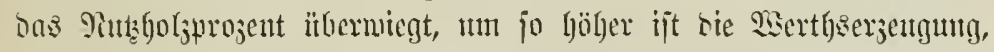

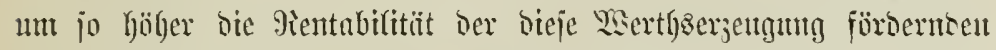

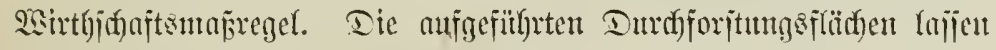

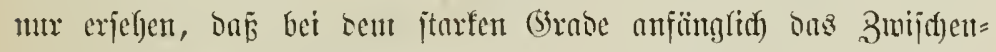

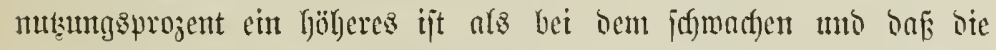

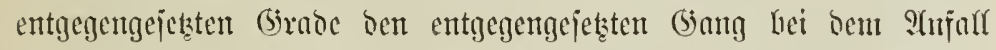
von 3rwijfentmetzungen beobadten, fie gewöhren aber feinen Cinbliff

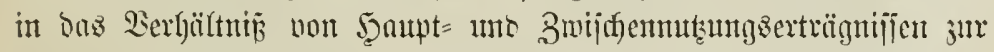

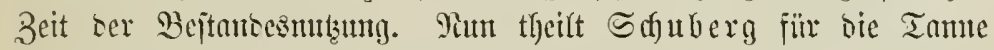

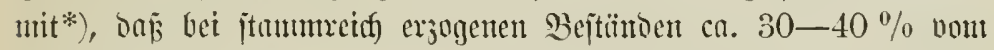

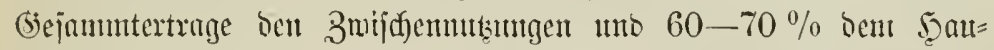

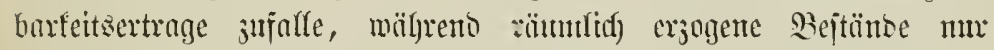

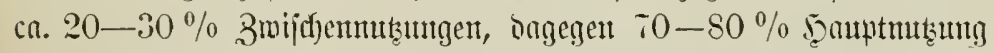

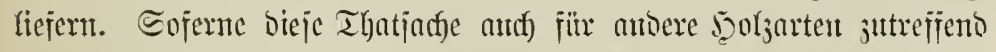

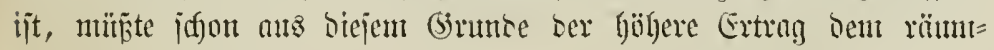

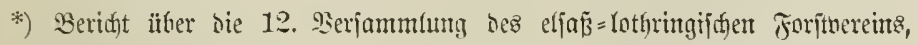
$3 \mathfrak{3 a r}, 188 \pi$, ธ. 23. 


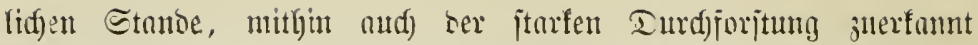
werben. Yllerbings mögen Llmittände lofaler uno fommerjiefler Platur

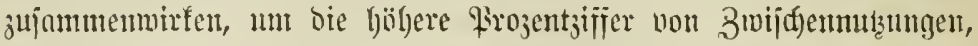

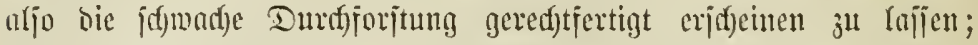

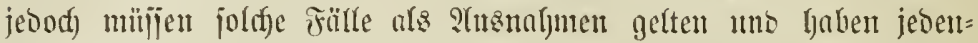

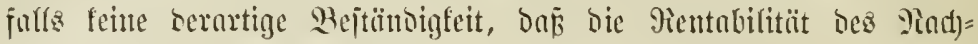
ljaltes bamit ju redjen ljütte.

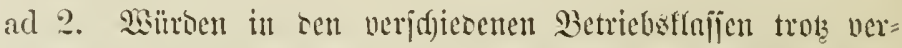

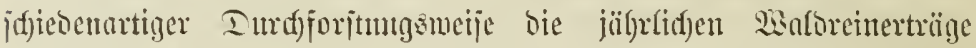

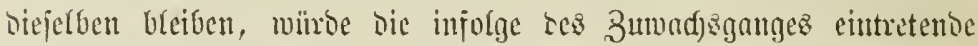

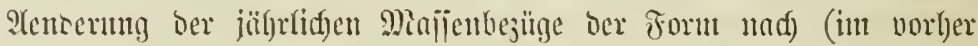

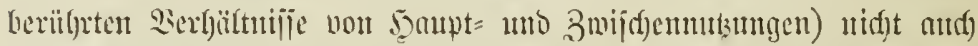
bie ?entoerumg ber sierthertrïge lesingen, fo wöre, ungendftet ber

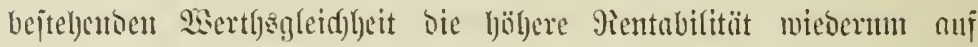

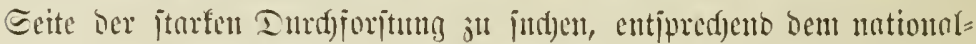

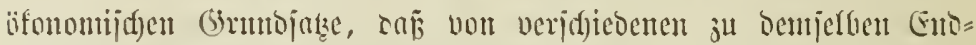

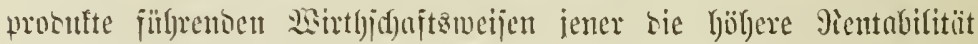
zuffommit, weld)e bas geringere sapital nujwenoet.

Bei ser Iurdjourfumg ïberlyaupt begimnt bie Sinpitalvernin=

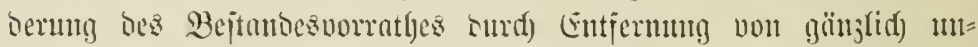
th)ïtigen ober un mit jefle geringen Bumad)sprojente urbeitenden Bejtanoesgliebern mit ben Eintritte einez Bejtandes ins burth=

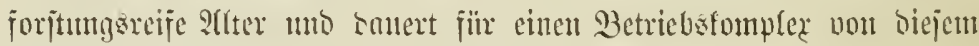
3citpmtte naleju bis jur beanborfeit an.

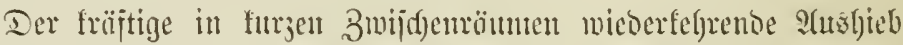

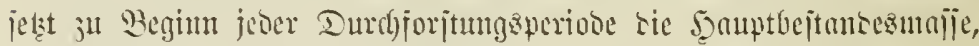
ons 2orratgs: mo Frobuftionsfapital, bes betreffenden Bejtandes

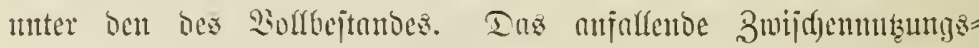

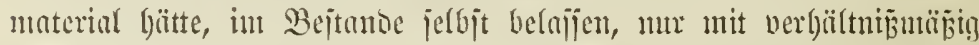

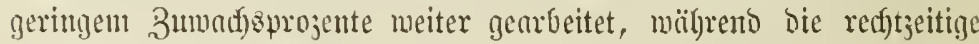

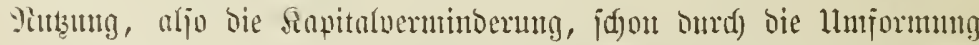

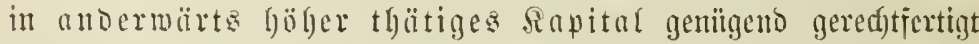

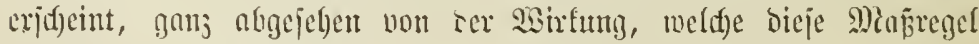
auf bie Buwadjatthütigfeit des verbleibenoen Bejtandes - rajajere

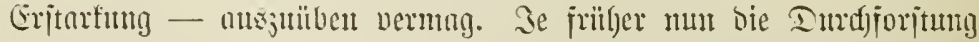

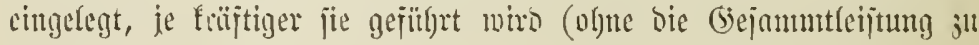




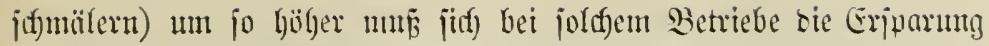
an Dem bie Pandfyaltigfeit gewïlyrleiftenoen Piormalvorrathe, um jo ljöher bie Pentabilitiöt jtelfen.

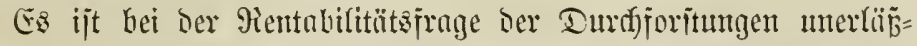

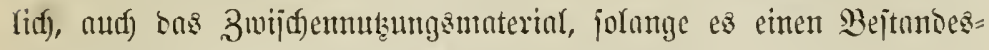

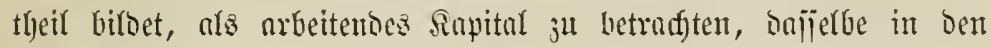
Diormatvorrath) einjubegreifen. Durd) Berzögermitg Der Dernus̆alyme

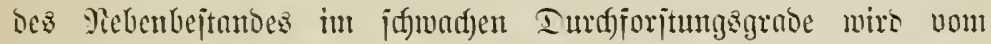
burdforjungsereifen Y(ter ab ein böherer Sapitaljtodf jur Frobuftion aujgewentet als im jtarfen Grabe. In ber Difijerenj des auj= gementoten Saptales liegt mut bie Eripartmg, bie mögliffe Steige=

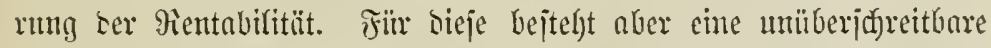

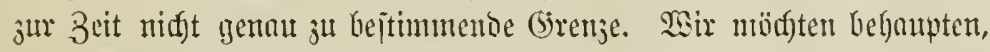

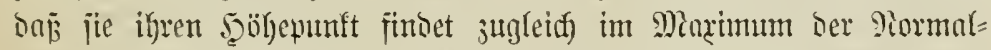

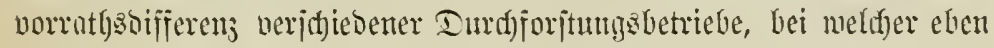

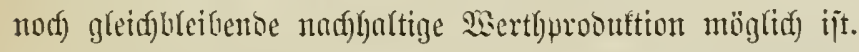

ad 3. 2aitjreno uns jür bie betoen eriten Mö̈glicffeiten ser

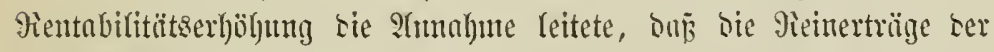

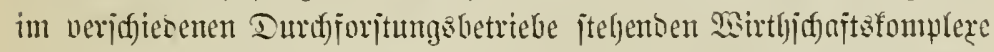

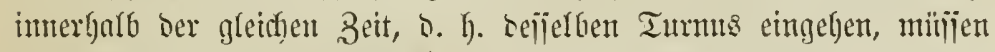

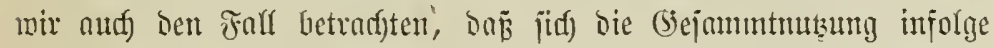

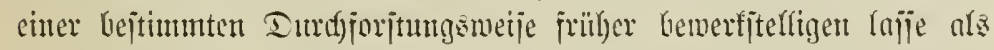

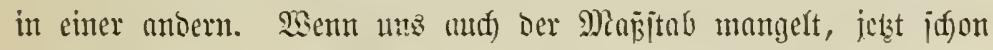

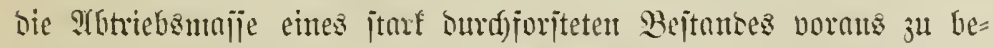

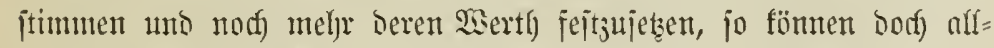

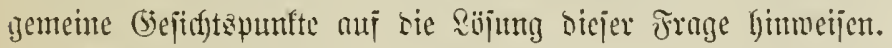

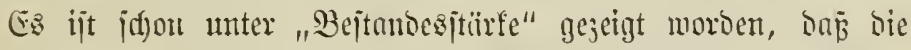
friijtige Durdjorjtung einer rajderen Bejtandeserjtnrfung 2 soriduth leijtet. Zumuadsprojent rejp. abjofute Zumadfägrübe uno Etammjahl

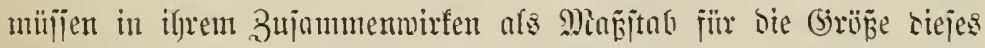

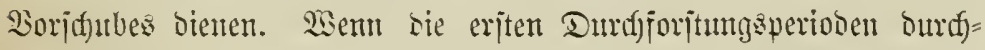

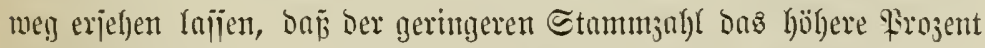

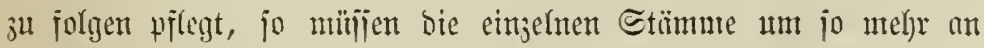
Etïrfe junelgmen, je geringer sie Etammjall iit, sie jith) in ben Zumadfs theilt. Serbit bie gleidfuleibence ober abnelynente abjohte

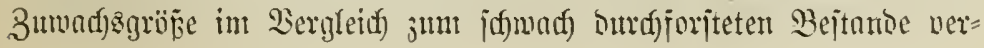




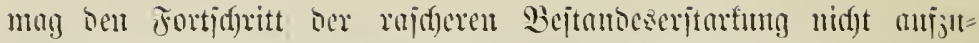

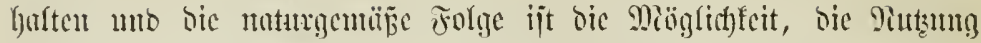

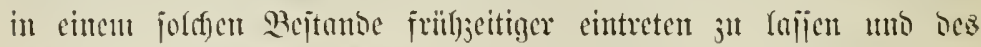

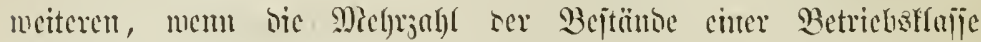

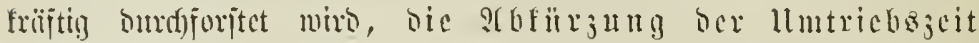

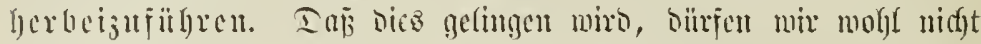

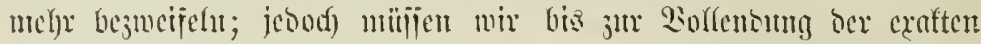

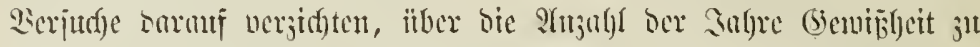

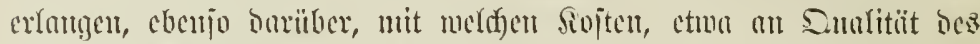

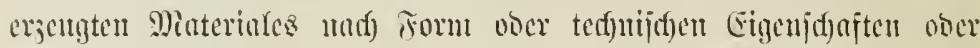

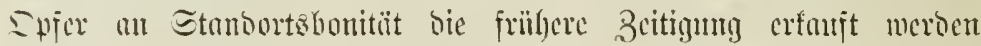

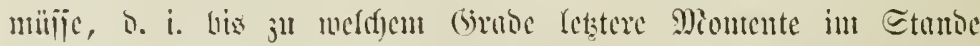

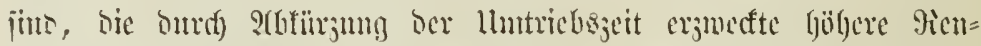

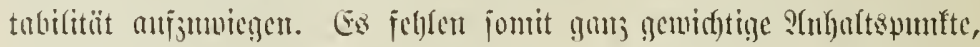

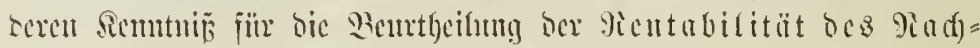
f) alt betric bes muntgüng(id) notguentig ijt.

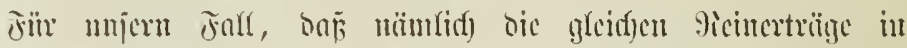

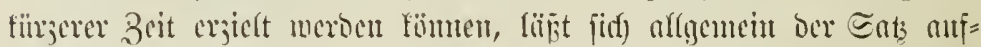

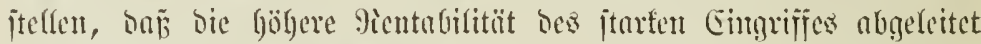

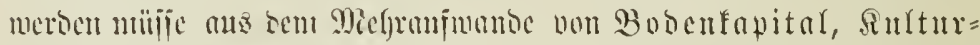
fojten=, Sermoltungstojten= mo Bejtundesfapital, ber bei

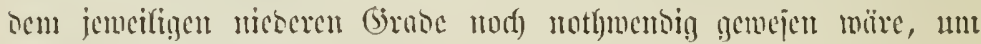

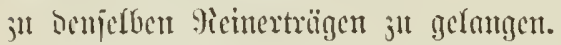

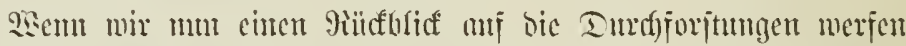

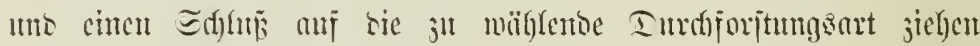

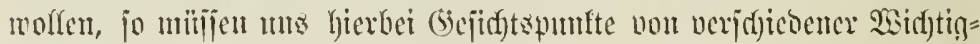
teit leitcr.

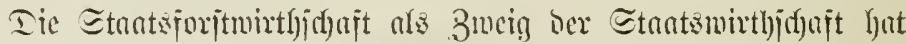

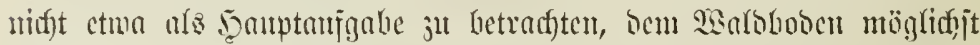

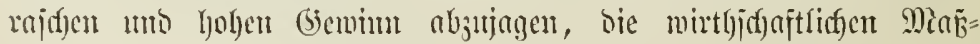

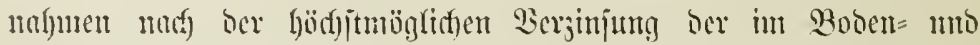

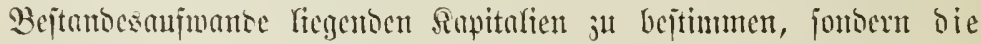

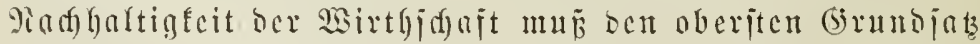
bifon uno crje im gialymen biejes brumbjatzes gat bie

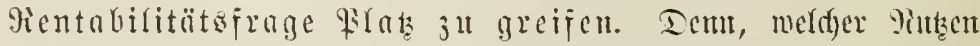

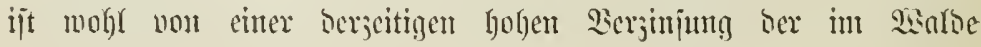




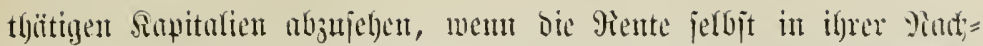

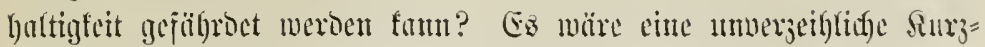

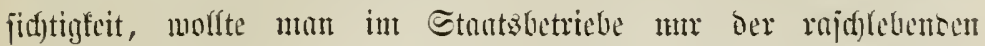

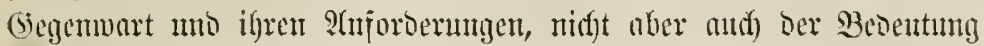

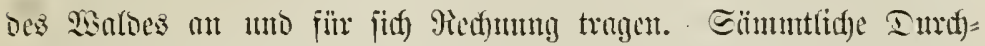

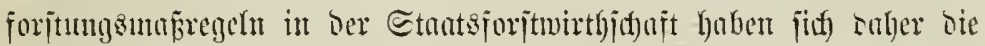

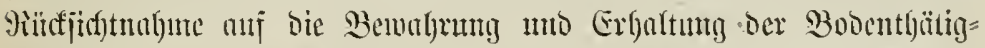

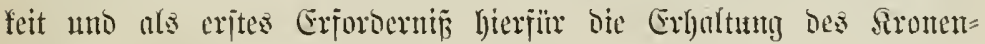
fithluijcz*) an sie Stime ju jidreiben.

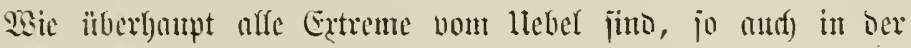

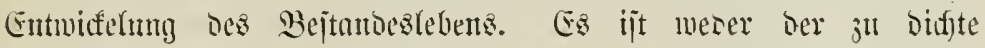

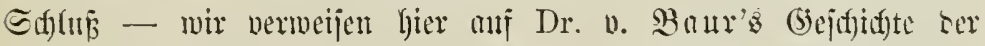

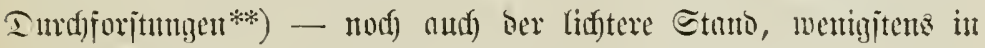
ber Sutgent, fïr bie Bejtundescntmicfelmy von sortgcil.

Es möge m biejer Stelle gejtattet jein, mi Den in Der Ein= reitung ungeocuteten Serglesid) juijiden oer Menjugeit mo bent

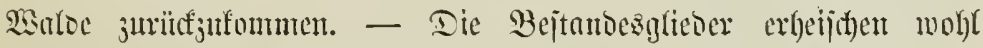

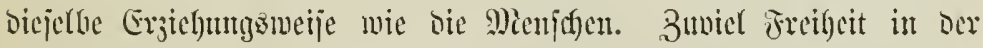

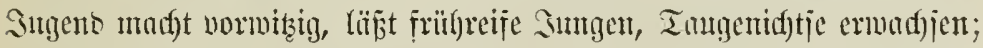

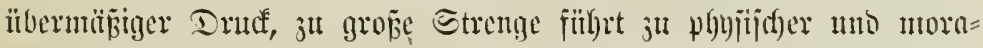

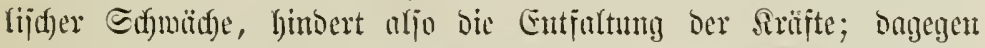

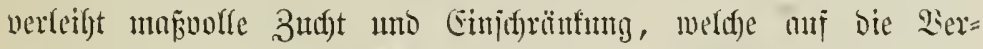

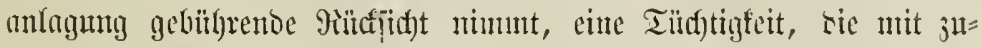

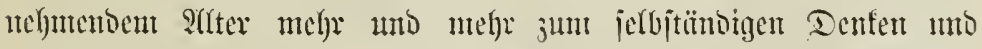

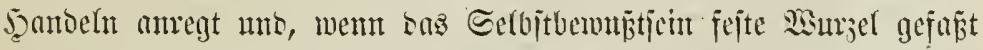

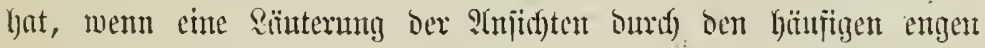
Berfelyr in ber Selt eintgetreten ijt, im borgej(f)rittenten ?(lter ju

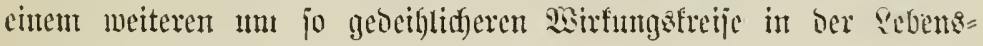
jterfung befiüligt.

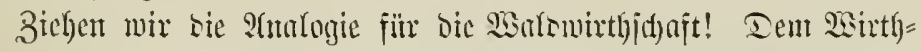

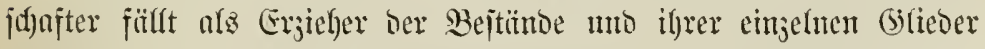

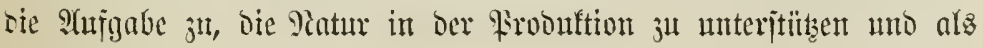

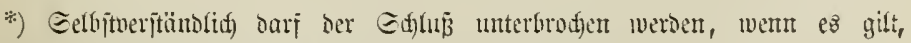

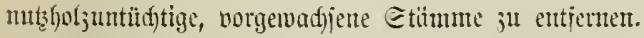

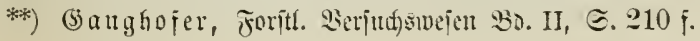




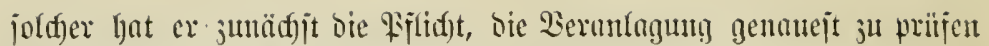
uno biernad) bie Erjieflumigametlore einjurid)ten.

Eomit verbietet es jidf) won jelbjt, eine bejtimmte Dur(t)=

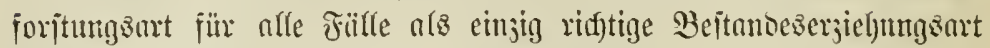

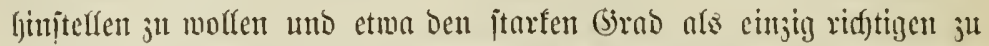

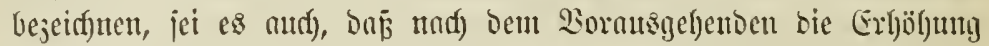

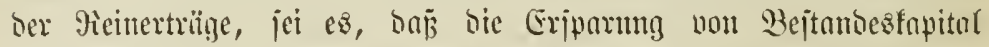

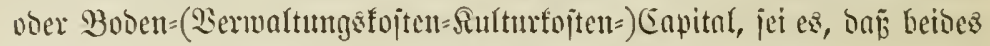

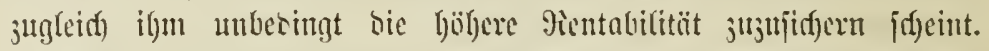
Edfmad) neranlagte 2inturen, Beptünbe geringer Bonitït verfangen cine idonentore, langjamer vorgelentoe Ergithung alz jente bejter Bonitüt (benen unjere ungejülfren Fläd)en entjtamment). Cis foum bci jdjledjter Etantortigüte Der jtarfe (brub, went er nady $D e=$

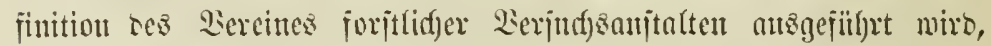

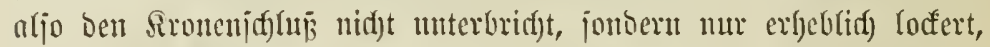

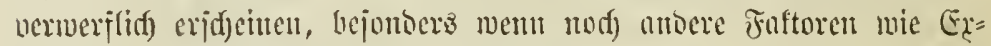
poiption bie Bejtonoeserjicfung eridjucren.

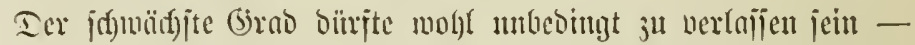

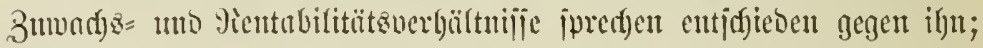
bagegen mag ber jtarfe Grab ba jeine Seredftigung fimben, mo

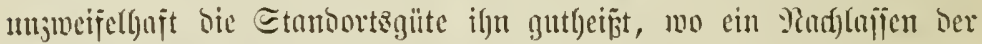

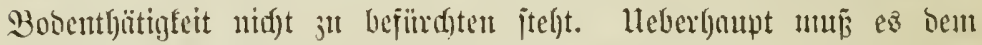
norjidftigen Etnatsumune gentïgen, bie Pientnbifität jtertty in ben

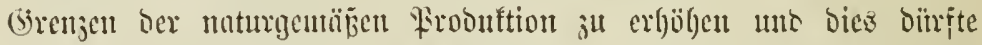

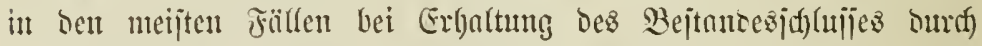

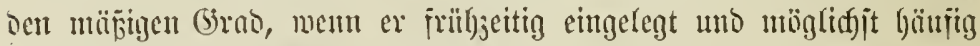

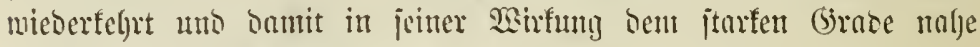
fonmt, in ber juecfoienficfjett rscije erreidjt wersen.

\section{Sicfifutugsbefxieb.}

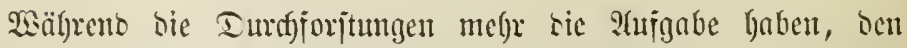

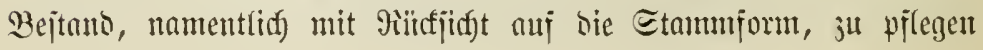




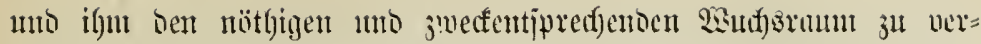

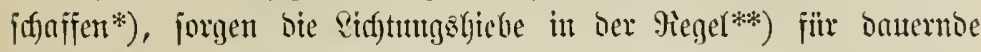

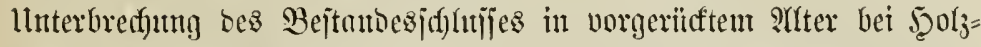
arteu uno auj Etmonten, bie mit Dortlyeil bie Fotenjinung bes 3uwarjjes in ben verbleibenoen Etümmen ermarten Kajןen. Hebrigens

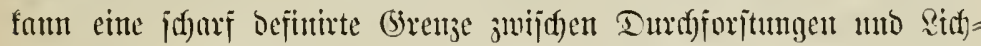

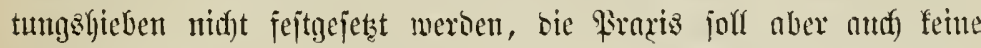

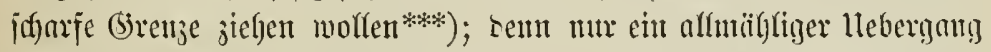
aus bem Durdforjtungsbetriebe in ton lichteren Stano berntag ju

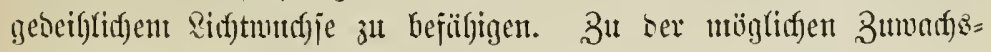

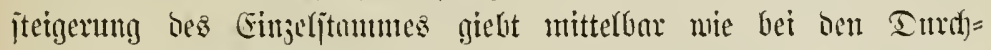

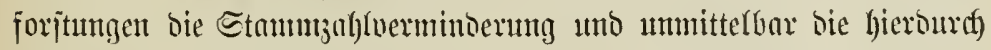

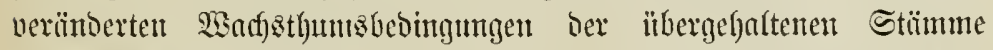

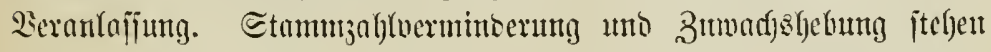

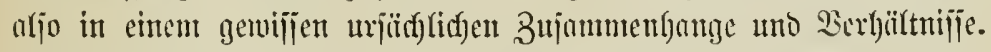

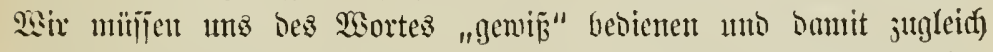

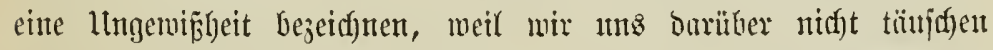

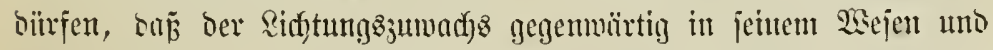

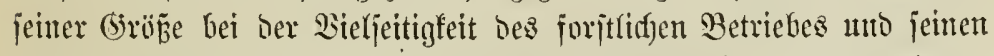
langen Frobultionşeitrïumen nod) nicf)t vorljtïnbig erfannt uno ge= mitroigt meroen fann.

\section{Emunadjagnน.}

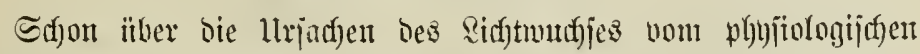

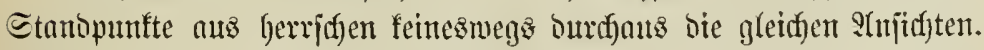
Desgletufen gehen bie Beoliadtungen ïher ben Beginn bes Sidjt=

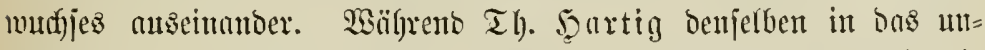

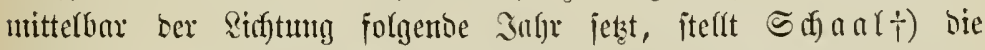

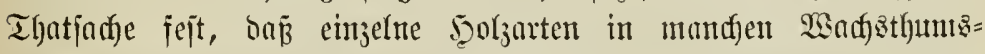

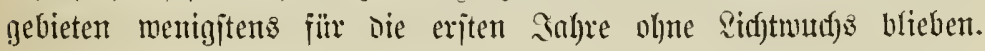

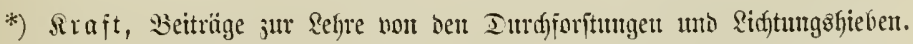
5̧unnower 1884, ธ. 16.

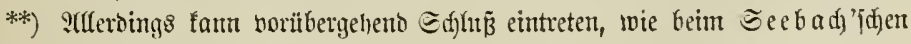
Betrieke.

***) Bayer, Maloban. Bertin 1889, ङ. 572.

†) Forftl. $\mathfrak{B r} .1876, \Xi 244 \mathrm{ff}$. 


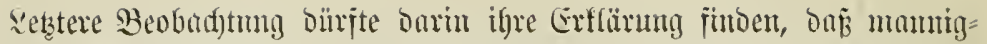

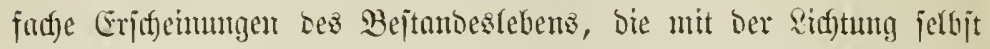

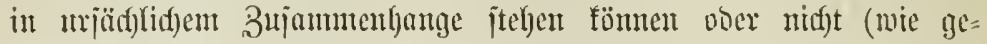

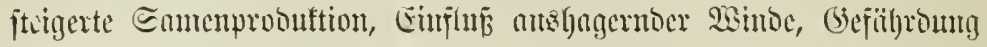

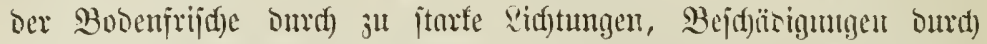

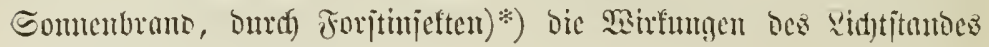
jut paralyjiren vermögen.

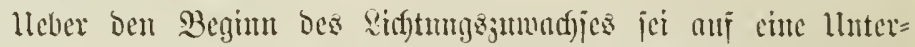

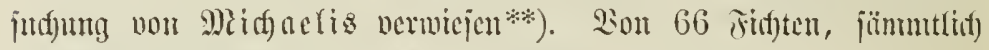
Der jtïrtiten Dominirenoen Stajie antgeböremb, jeigte

$$
\begin{aligned}
& \text { im i. Salgre madf ber vidfuturg } 8 \%
\end{aligned}
$$

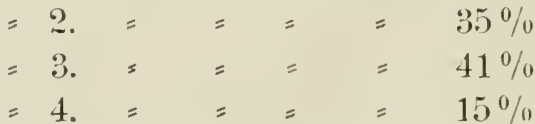

Den Begim ber Salfrringueroreiteruty.

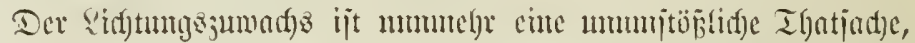

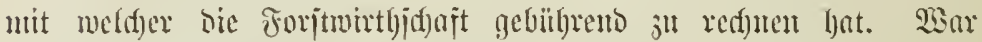

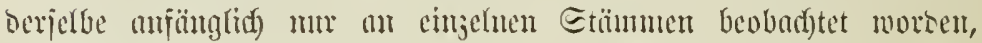
jo trad)tete man balo burd) bejontere Dicbzoperationen ben Pidft=

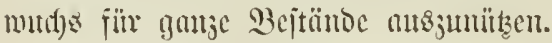

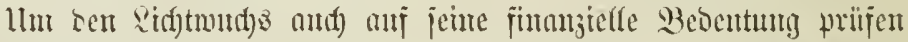

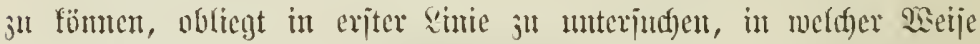

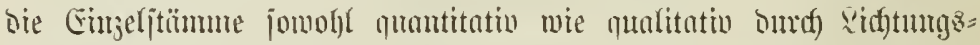

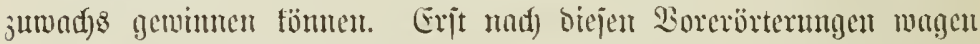
mir as auf bie Betruftumg gamjer Beitünte, bie biejem Betriebe unteriteflt jinto, je nad) ser $\mathfrak{A}$ rt, wie jie inren 3 wedf verfolgen, ein= jugeben.

\section{A. Siditungsjumatis an Einjeljtanme.}

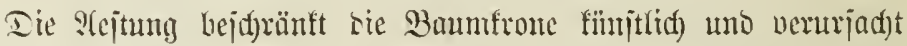

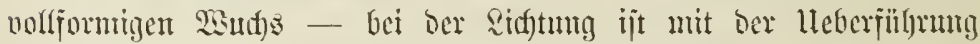
Des Etanme in ben freieren Etand bie Afustreitung oer Fronte ver=

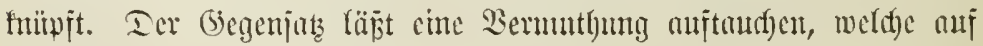

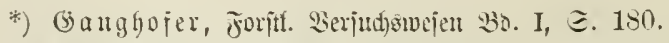

њ) Joritl. Blätter 1884, હ. 186. 


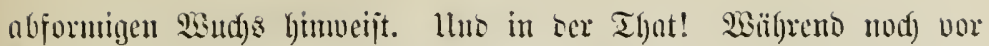

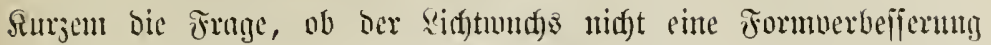

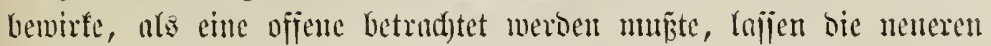

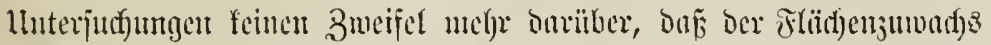
bei fidftgejtellten Bäunen nou ber Spitze nad unten ganj beseutend

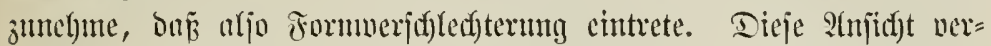

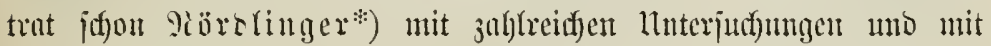
benjelben jtimmen im mejentliffen ïberein jene nou Borggrene;

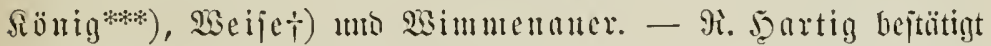

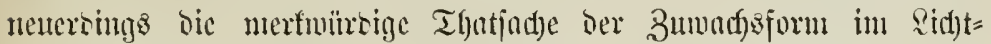
ftnmbe an juei Budfen, meld)e nad) 7 jüfriger Freijtelfung cine

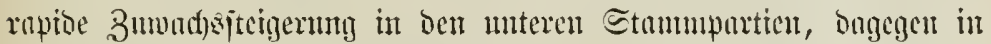
bor Bannfrone etmas Sinfen jeigten. Sie plyjiologijde Exflürmy

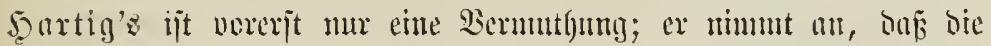

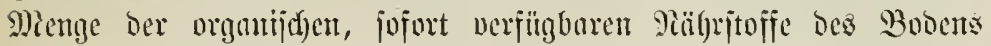

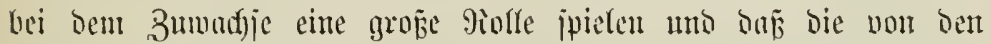

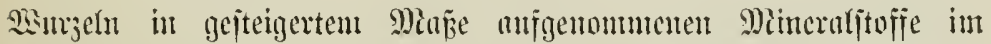

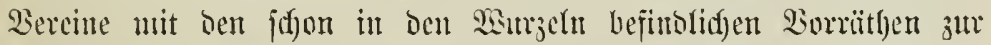
Erjentyung bon Eimeipjubjtanjen um fo med) bettugen, ie nïber bie Eambrultegion oen $2 \mathrm{sum}_{\mathrm{j}} \mathrm{eln}$ jelbjt gelegen ijt.

Dieje (f)mofterijtija)

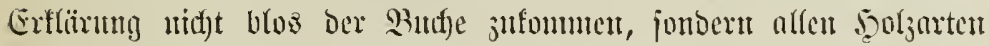
mefre voer meniger eigentlümulidf jein.

2uf Seite 58 ijt jdematij(h) eime Darjtellung ber Etamm:= analuje ciner Fid)te $(328 \mathrm{~mm}$ jtarf), Fïfre $(426 \mathrm{~mm})$ und Tanne $(379 \mathrm{~mm})$, die แแะ bent Foritnute Freijing, Dijtr. Lnt. Forjt,

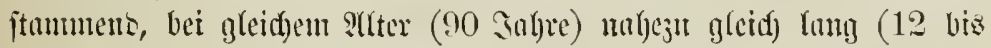

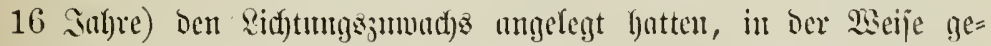

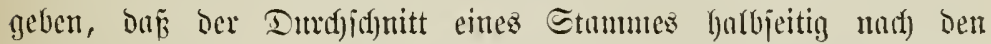
mittleren giobien ber cingelnen Seftionen für Sandfopertuben won

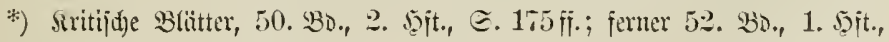
હ. $154 \mathrm{fi}$.

**) jorjtl. Brätter $18 \pi$, ๔. 215 .

**) Forftl. Blätter 1882, ๔. 361; 1886, ๔. 46.

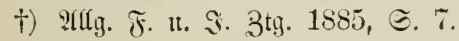




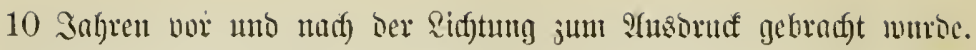

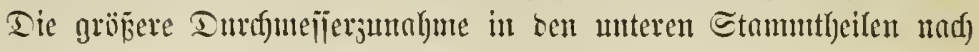

Fig. IV.

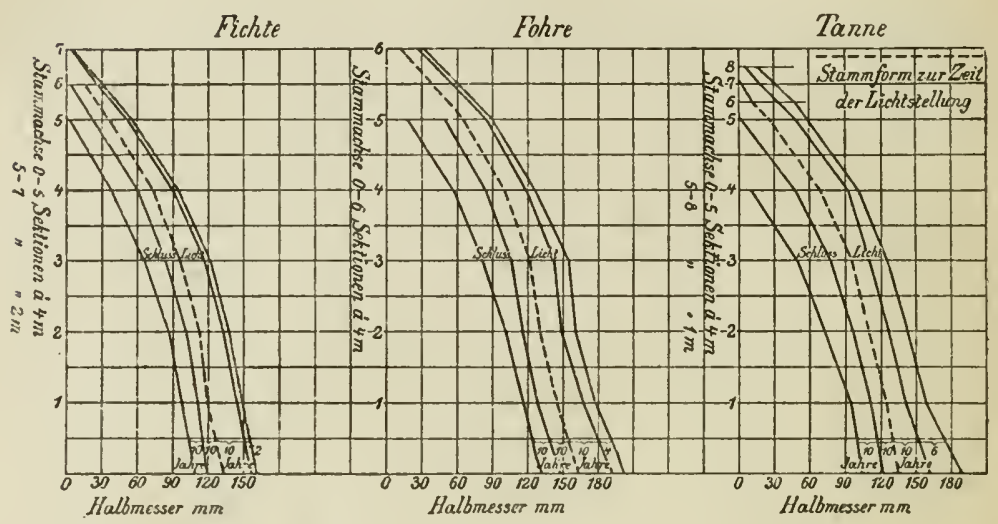

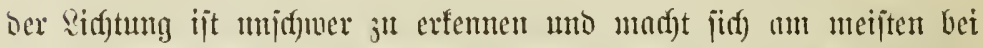

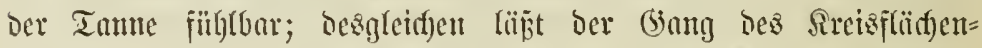
jumadjes in ben cinjeften Etmumpeftionen bie Wrirfung ber sidftung Beutlidf) herwortreten. Iie nor ser Sidfutung int Follen begriffenen

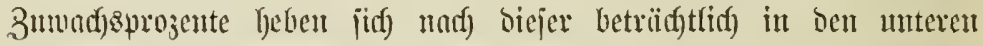

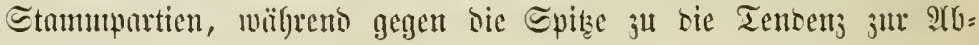
nulgute herrifgt.

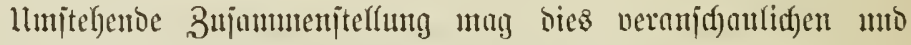

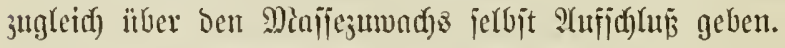

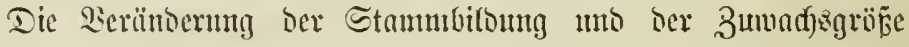
injolge ber sidftung birrfte in ben woritehenten wenigen Beifpielen

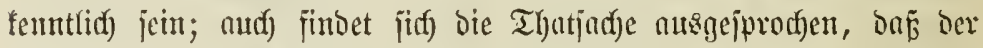

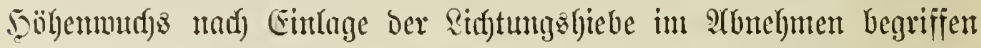

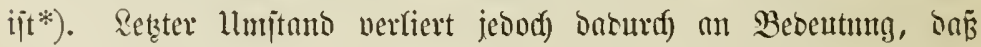

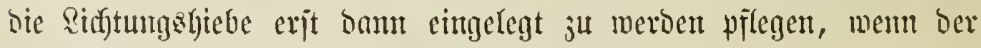

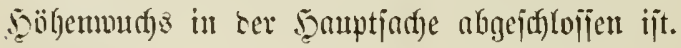

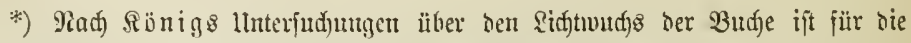

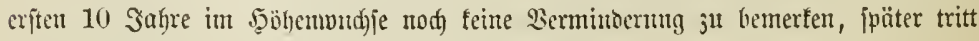

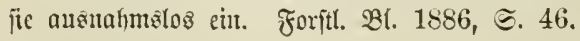




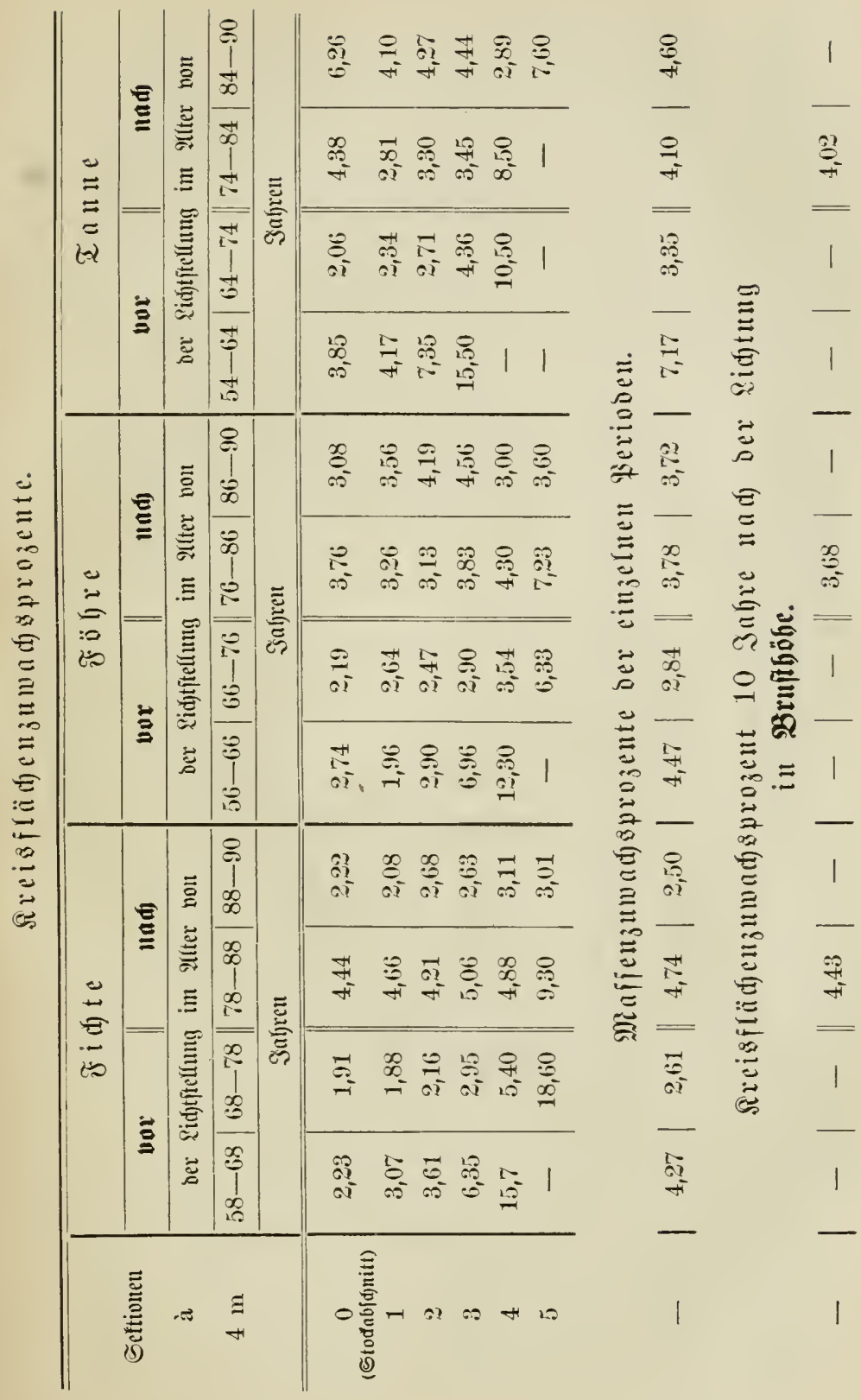




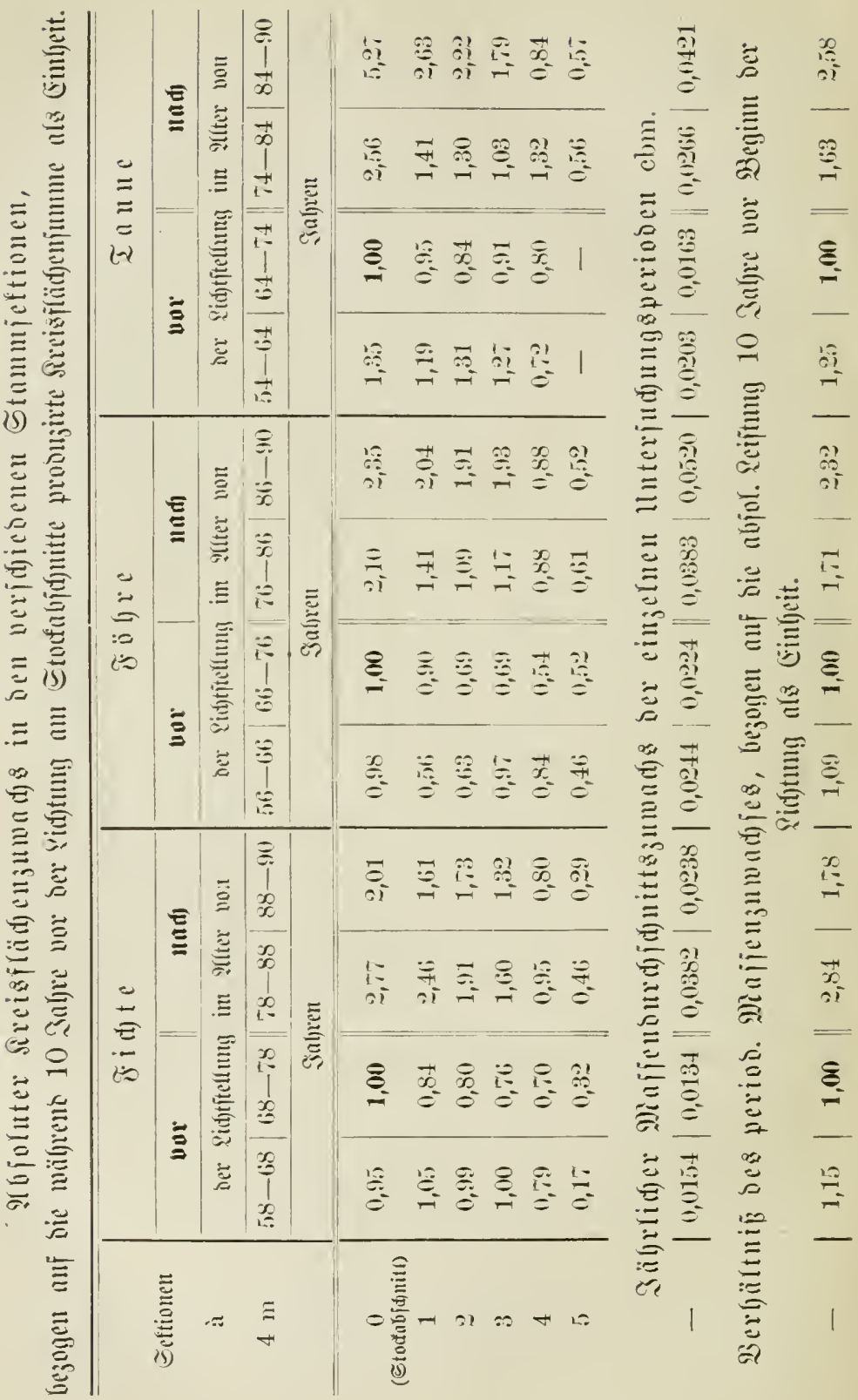


Das Minjejumadfostrojent juetut bei ber Fidfte in ber erjten

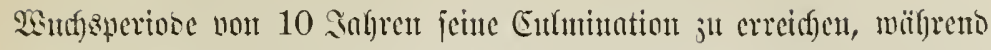

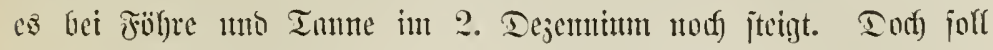

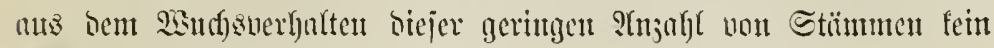
altgenteines bejets gefolgert meroent.

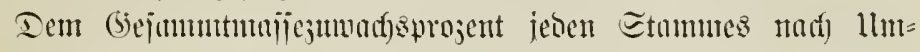

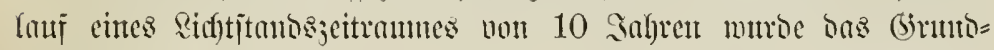

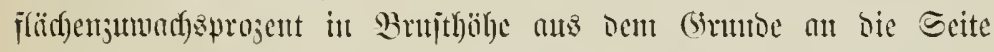

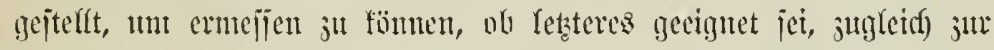

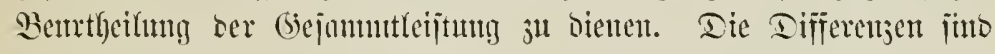

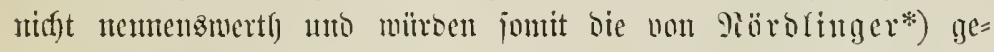

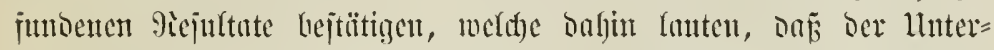

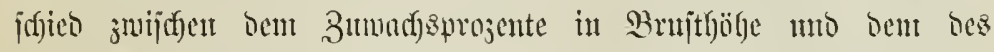
ganjen Stamunes mührento tes criten uno jueiten Dejemnims ber

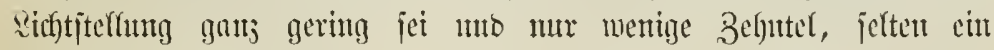
ganjez Pirojent erreiffe.

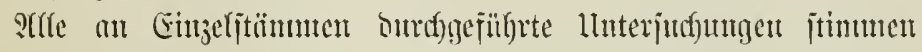

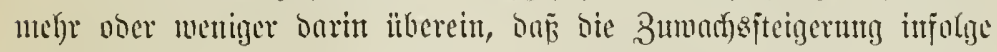

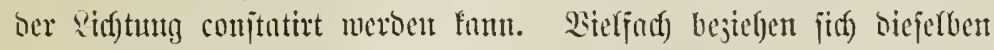

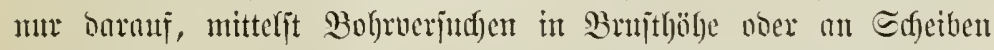

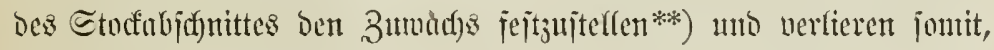
ba fie itber bic (jejanmtleiftung feine Sidferbeit gemälfren, eintiger= mağelt an $\mathfrak{w e r t h )}$.

Die anf ङ. 60 mitgetheitten 3 afden geben bejitglich Der ab=

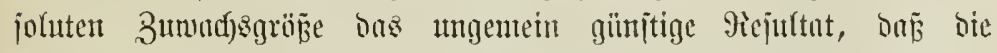
Sinzelitïnme im lidfteren Stmbe melyr als Dos Ioppelte mie un= mittelbar vor ber eirftutng ju probujiren vermögen. Sönig theilt

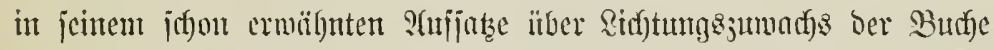

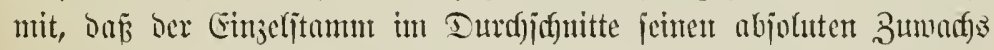

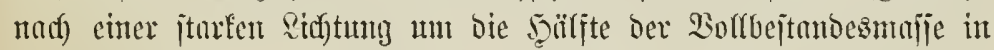
ben erīten 10 Jaffren mod nidgt verboppelt, mähreno bies annäherno in ber folgettoen 5 Salfren gejfjielyt.

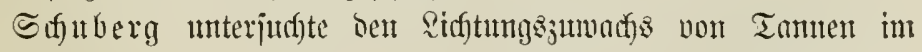

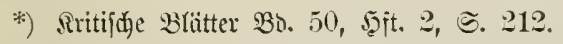

**) Danderm., 3. F. F. 1887, ธ. 50; 1889, ธ. 3. 


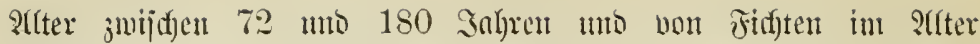
jmijajen $75-135$ Satjren uno fanto*) bei $10-30$ jïfrigem Heber= halte, bon bas Zumaffasprojent mit Zutalyme der Baumbühe, mit

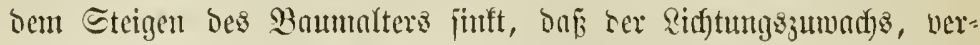
glidjen mit bem Zumadjic des geidjloijenen Bejtandes, nodymals

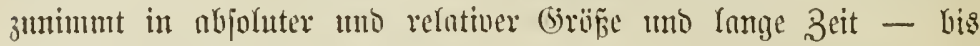

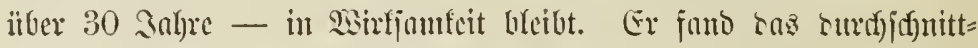

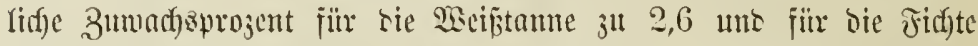
magejut von gleitger 5oühe. (Bei ber Tame mar bns Steigen fofort madf) ber \&idftung cin Yebfoftes.

Fith sic Siejer fano Dr. Storp cinen Sifftungsjumadfs, ber

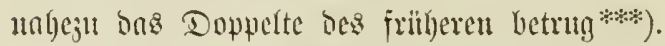

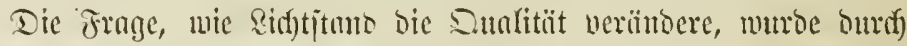
bie bisherigen llnterjudfungen meijt ginnjtig beantmortct. 9eöro=

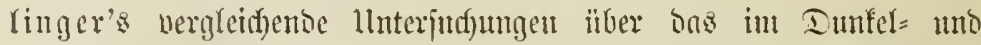

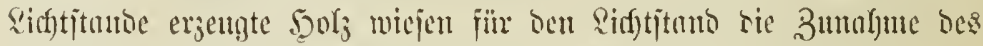

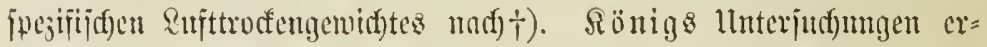

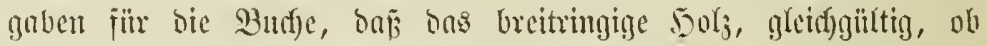

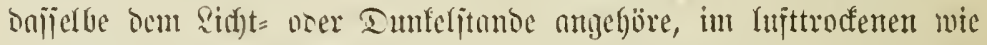

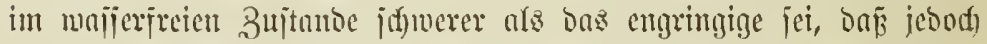

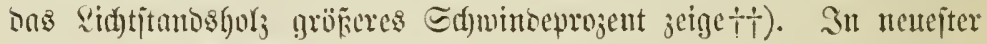

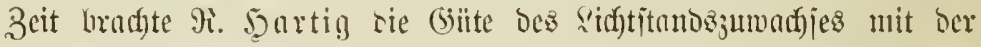

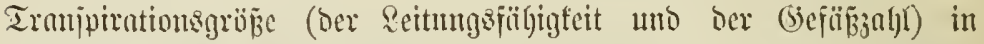

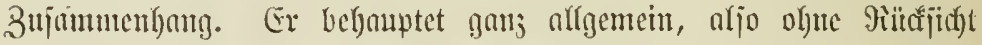

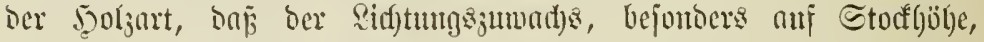

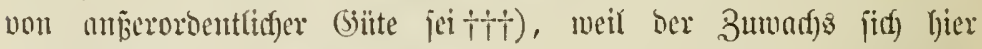

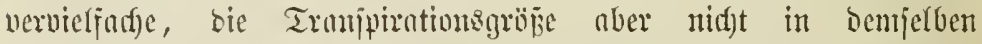

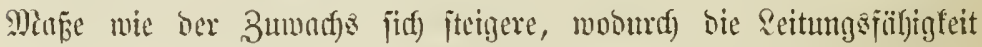

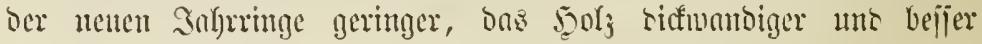
werte.

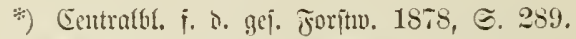

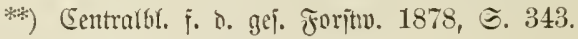

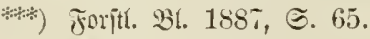

†) Sirit. Stätter 4i, 3̉. II, S. $56 \mathrm{ff}$.

†) Foritl. Bł. 1886, ๔. 50.

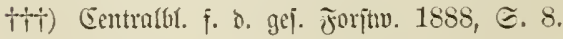




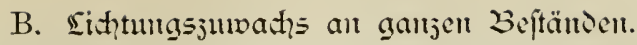

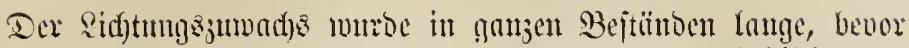

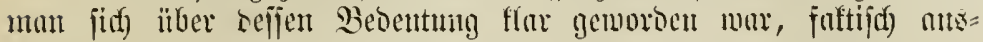
genutict.

Der Mittefwarbbetrieb jelbit, wo er bem Stamoorte nade an=

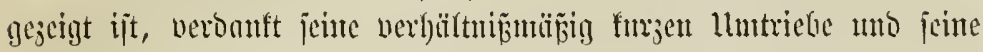

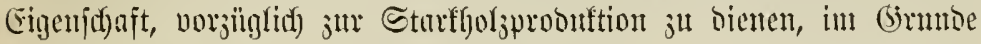
genomment oem Richtitanoe bes Soertyorjes.

Forner mus in lleberfalthetriche, ïberfonut in jümutfidfen

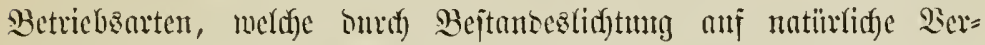
jüngmty abjielen, je nad) 2 serjüngm

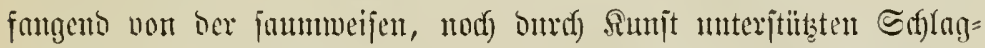

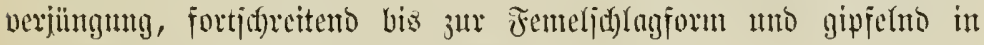
oer silenterform - eidftwudds fül)(bor weroen.

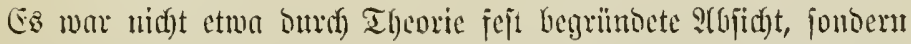

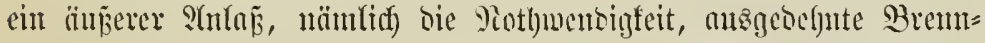

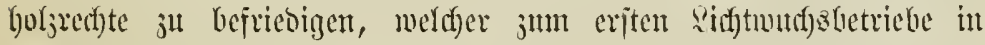

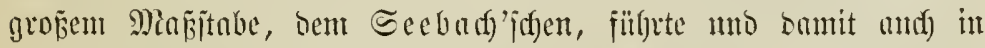

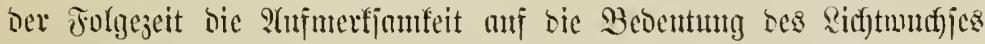
fiil ganje Beftïnto lenfte mo ju befien Ettoium anjpornte.

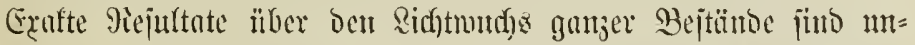
gentein jpürlid) unto bie wenigen vorffantenten mungels vergleidfonrer

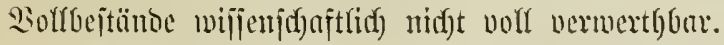

Dis Sdjwierigfeit, bent normitrfigen (jegenjtnno in jeiner ganjen

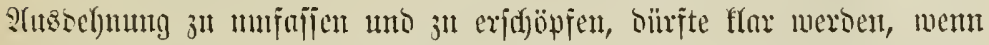

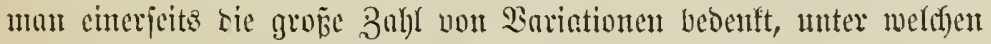

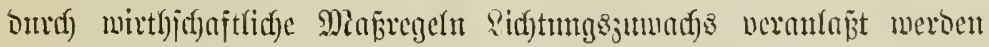

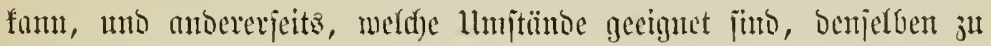

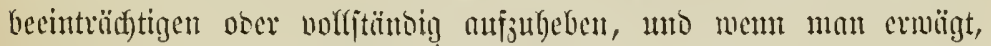

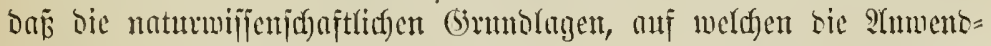

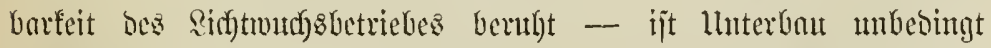

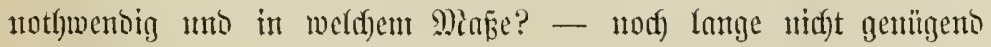
erforictit fint.

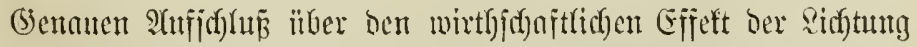

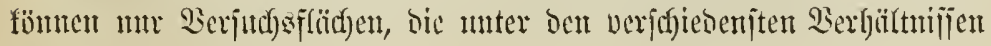




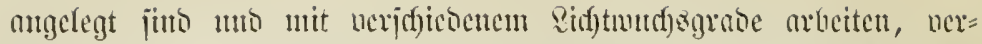

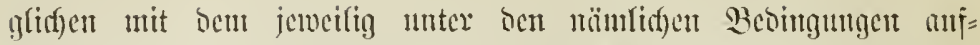

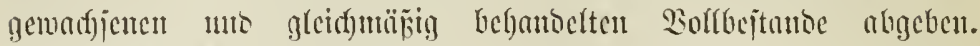

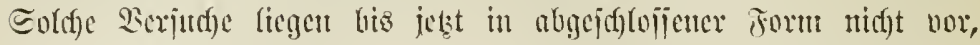

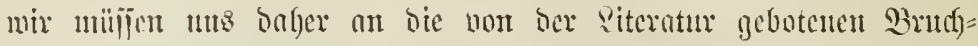

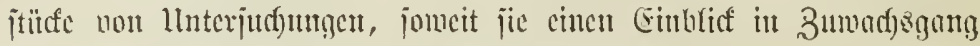

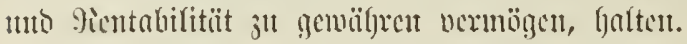

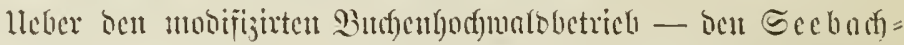
jefen agcuant - theift Sherforituteifter Sirajt intereijante, alferbings

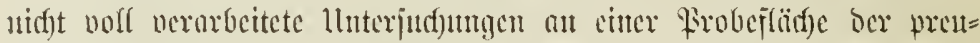

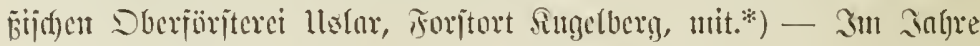

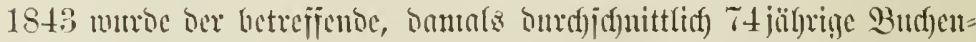

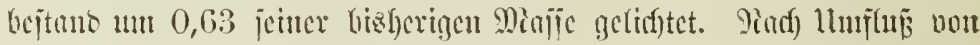

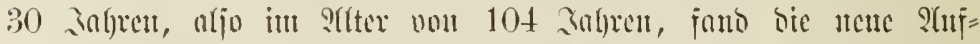

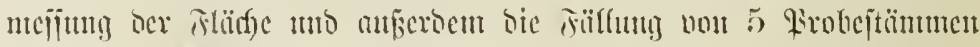

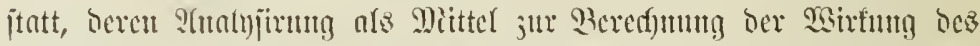

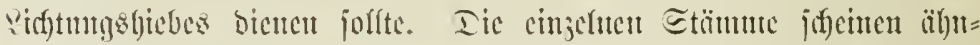

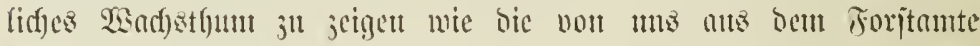

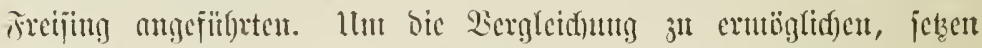

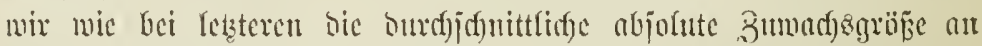

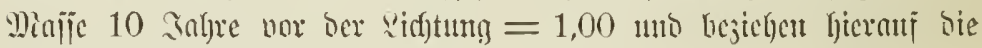

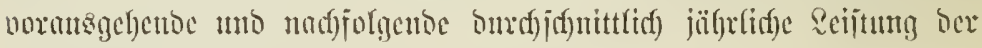
10 jü̈rtigen Weriosen.

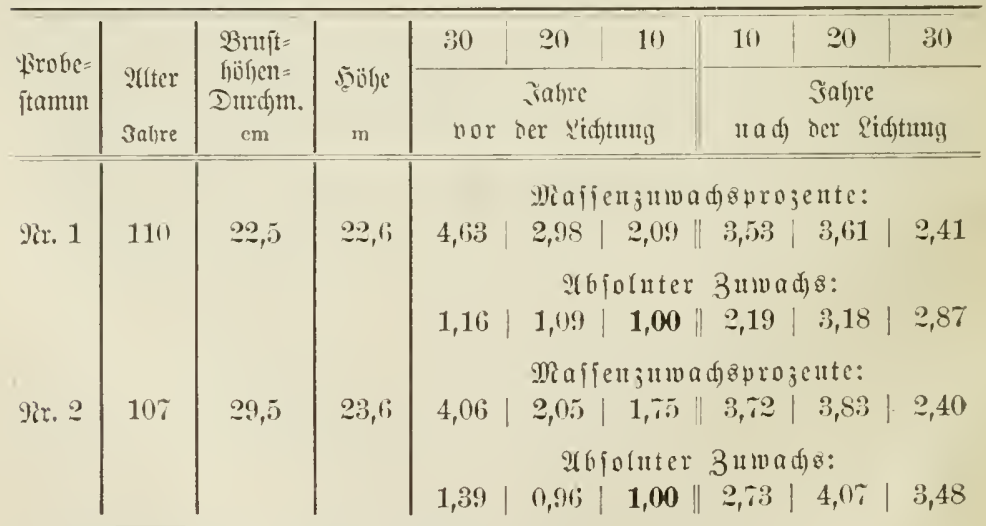

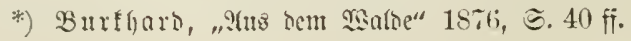




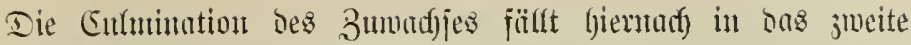

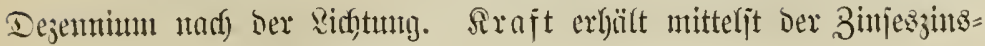

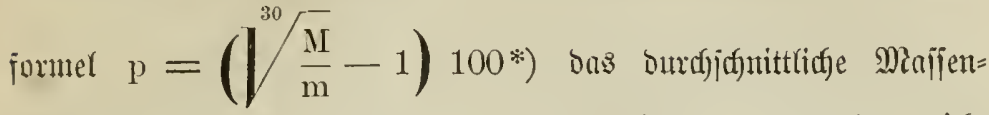
jumadgaprozent fïr 30 salfyre $=2,9$; mïfrento er fïr ben midgt mobifizirten, noflen Sot gleidfer Dualitüt Das Bumadjsprojent ju 1,44 amniunt. Die Sojten ber Begritnoung Dez llntermutajes, ahs

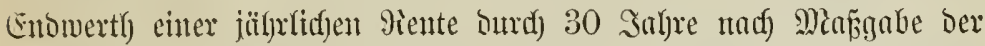

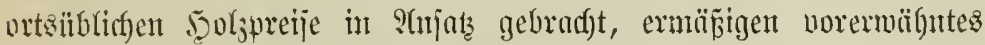
Wrojent 2,9 auf 2,7. Die 9ftsfiebsmajie 0,67 ber urfuringlidfen

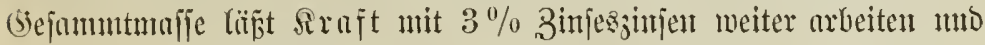

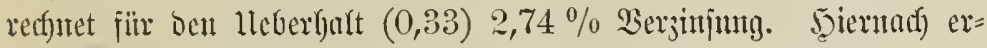

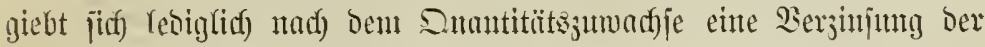

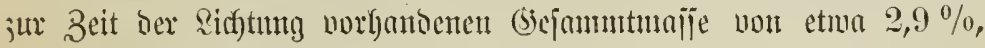
oafer doppelt foniel als jene des Solfuejtumbes betrïgt. Llebrigents

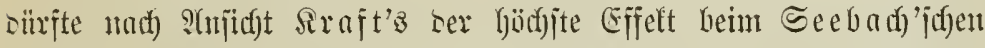

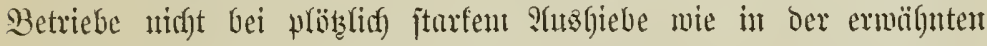

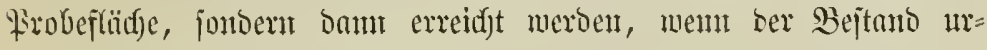
ipriinglidf meniger lisft gefaltent, in ber Mitte ooer am Beginte bes

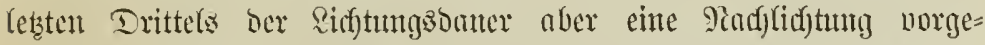

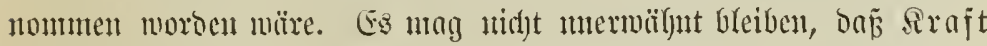

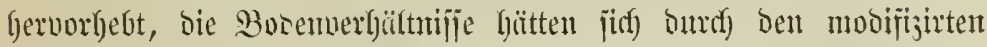
Betrieb gebeijert*\%).

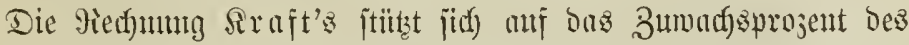

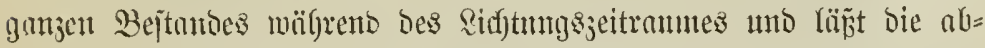

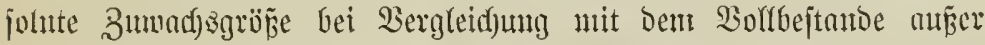

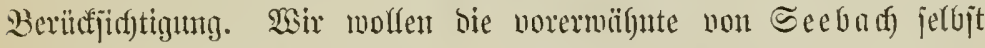
angetegte girobeflüdye $(0,56 \mathrm{ha})$, fomeit biez möglich ift, auf bie

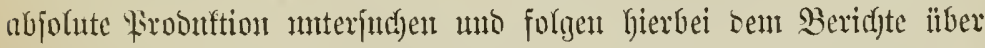

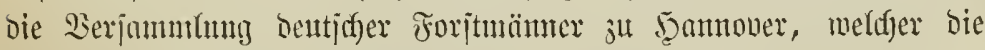
menejte Bejtantosonfungme enthält.

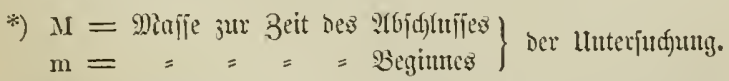

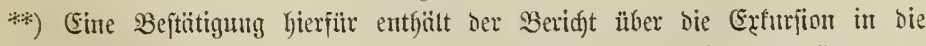

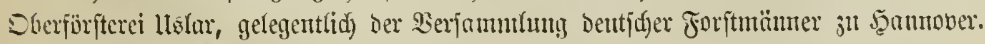
Beriaft ïfer bie Serfanmlung 1881, ๔. 175. 
Es betrutg pro ha in Salje

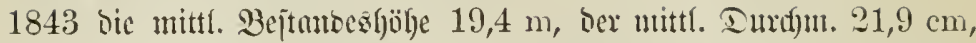
Der ?tusfieb 169,2 fm, ber leberbult 101,3 fm Derblyoly in 282 Etümmen mit 10,6 ym Stammuruncf́lïf);

$1873 / 74$ bic mittl. 3ejtancealjölye $23,4 \mathrm{~m}$, ber mittl. Dur(f) $32,5 \mathrm{~cm}$,

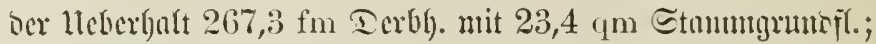
1880/81 bie mittl. Bejtunbeshöbe $24,4 \mathrm{~m}$, ber mittl. Durdym. $36,1 \mathrm{~cm}$, ber lleberhalt $279,6 \mathrm{fm}$ Derbfols in 223 Stümmen mit

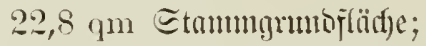

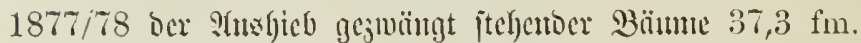

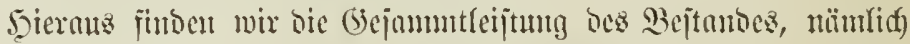

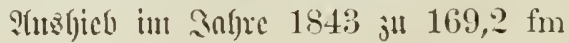

$\begin{aligned} \text { Borraty } & ==1877=37,3= \\ & =\frac{1880=272,6=}{\text { Sa. } 479,1 \mathrm{fm} \text { Derbubly. }}\end{aligned}$

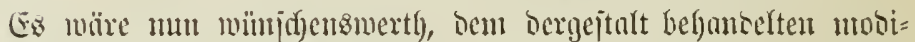

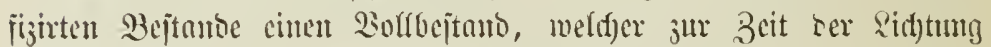
genan biejelben Bejtantes mo

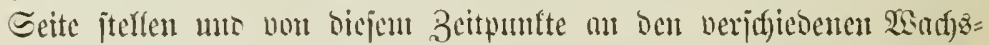

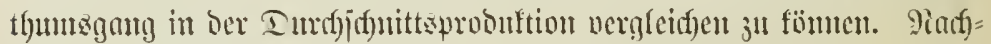

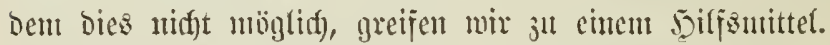

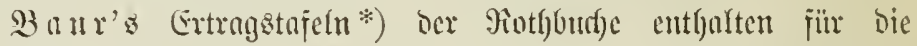

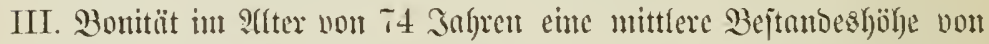
$19,7 \mathrm{~m}$ und eine Ierbutajie von $290 \mathrm{fm}$ pro ha. 5jöbe uno Borratf)

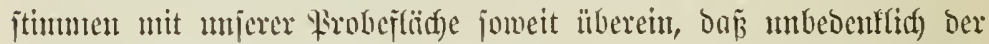

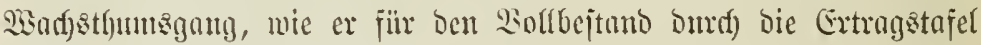

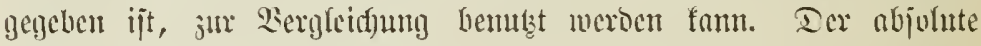
3umadis fïr bie sisudfoperiode

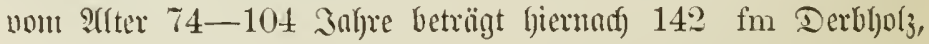
$=104-111$

ber 2 solfbejtumb im 74jülrigen sflter lyatte $272,6=$

jontit Zeijtung Des Bolfbejtmoes 44\%,6 fm Derblyoly.

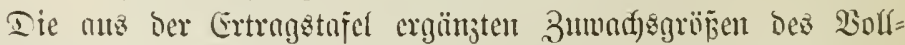

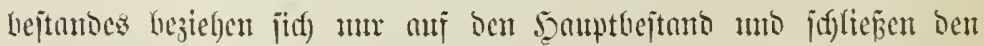

*) Dr. n. Baur, die Miothbutbe, B̉ertin 188\%, ๔. 103. 


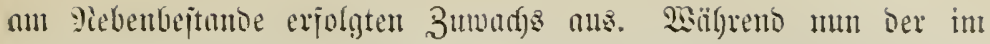

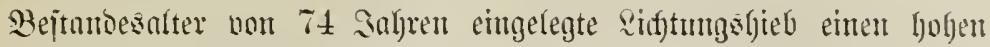

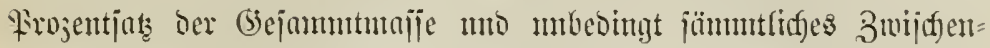

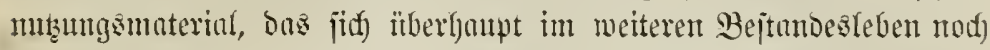

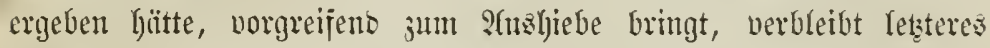
nod) längere 3eit ben 2ollbejtande und gelangt, jelbit nod) an 3u=

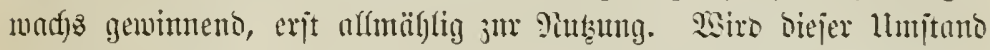

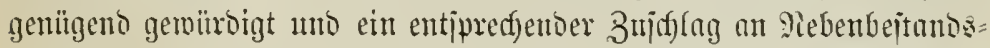

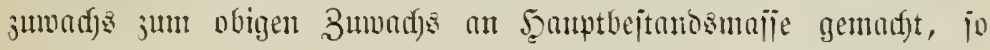

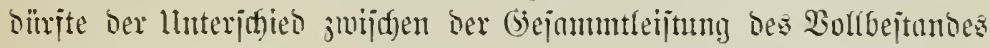

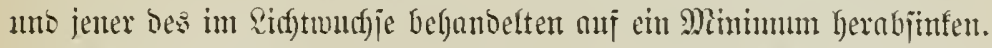

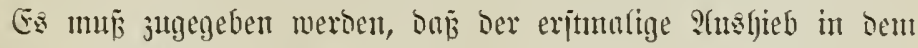

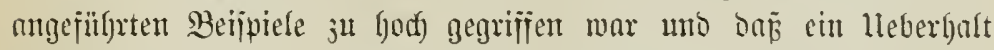

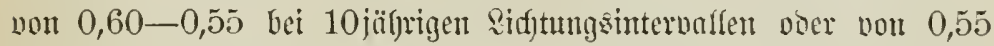

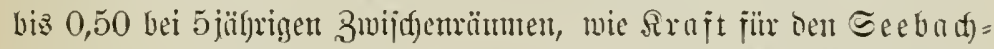

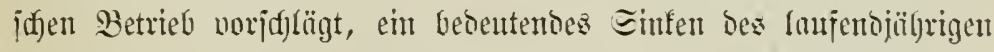

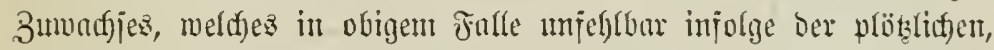

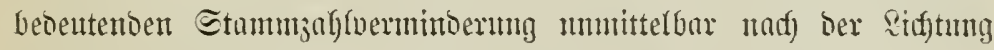
eintreten mup̆te, bätte verringern fönten. Ḑiermit märe eine jebmg

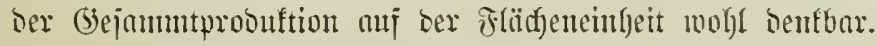

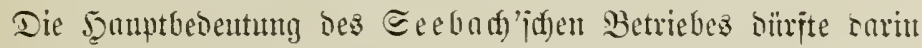
3̆ juffen jein, baż bie @tanmomenjionen bas Bollbejtundes in fitrjerer 3eit oner innerifalb derjelben Zeit rie jtärferen Dimenjunen

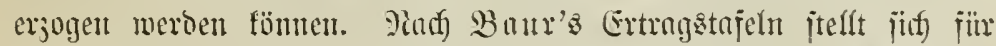

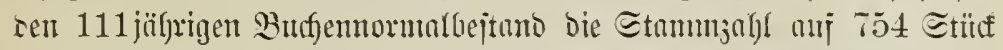
uno Der mittlere Iurdfmej]er malyu nuj $26 \mathrm{~cm}$, wrïgrent fïr Den gleidalterigen 37 saljre in Sidftitande bermofidgen Bejtanto bie

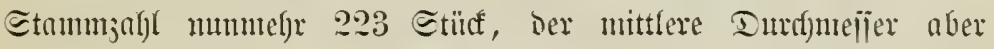
$36 \mathrm{~cm}$ betrïgt. Der Dmefjidnnittabaum Eer Ertragstajel bält

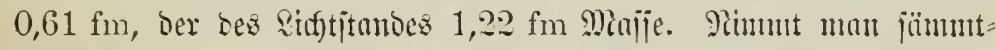
lidfe Ertrïge bes rithtungsbetriebes einerjeits (sas Materin der

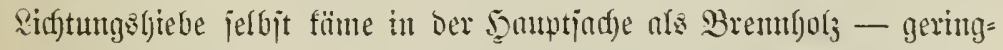
mertfig in Giedumng) und bes Bollbejtandes antererjeits jur gegen=

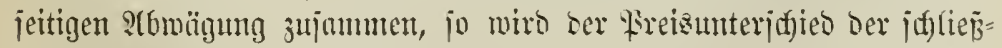

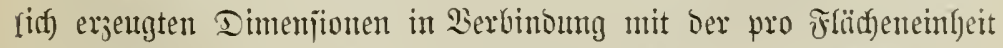

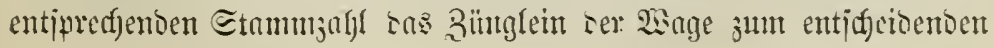




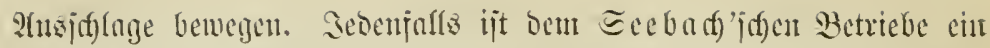

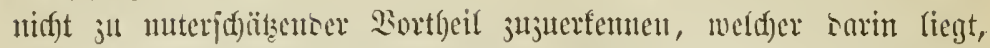

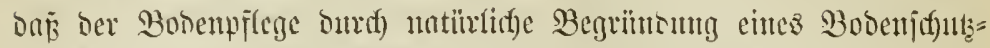

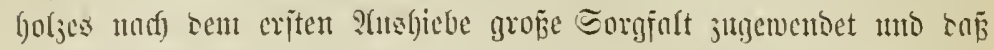

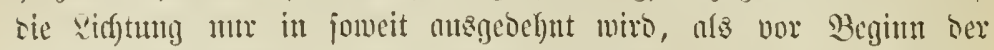

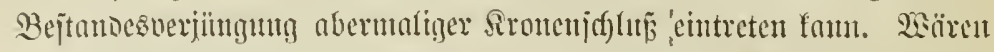

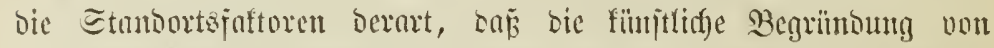

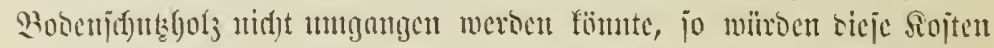

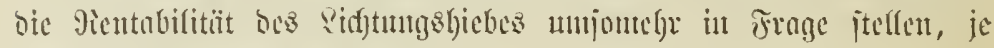

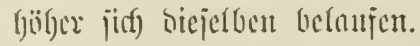

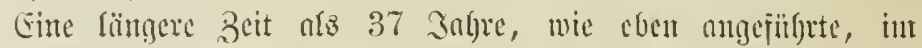

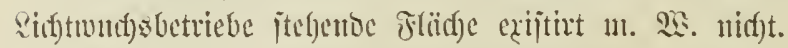

Fiin einen 115 jülfrigen Butc)enbejtonto mit geringer Eidfen=

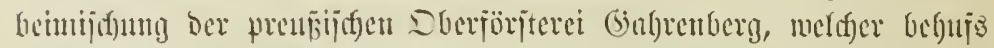

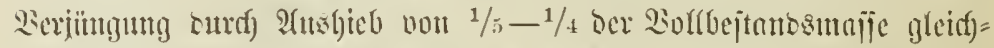

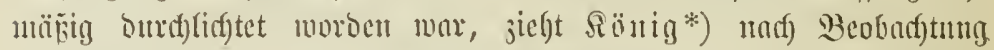

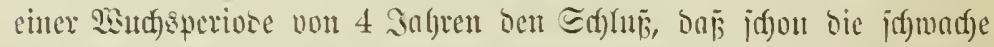

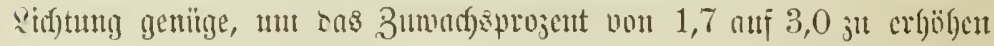

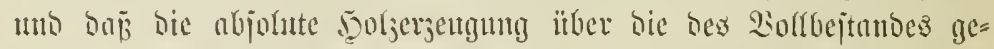

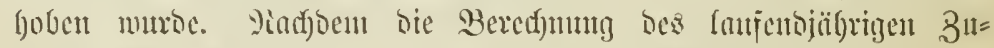

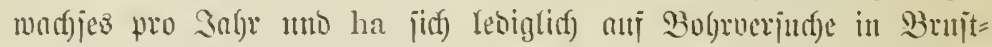

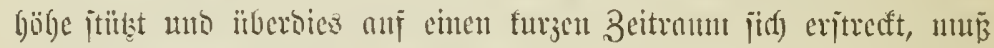

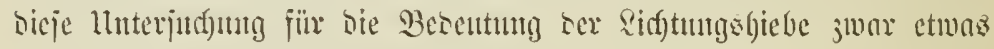
werlieren, ueroient aber inumerfin Bendftnty.

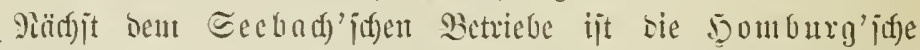

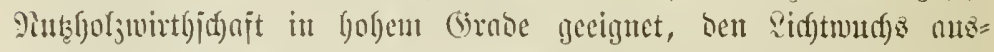

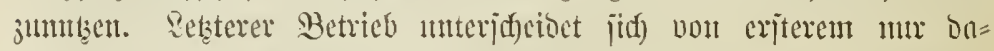

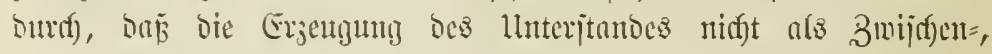

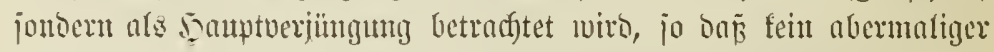

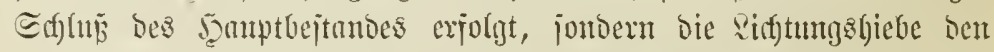

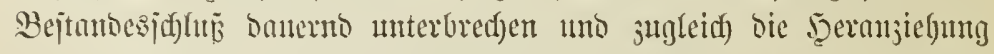

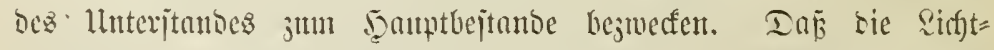

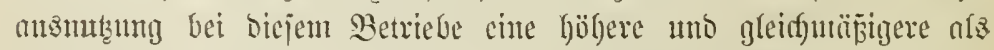
beim Eeebad'ighen ijt, mus non vorne berein anerfannt werben.

*) Forjt. B(ätter 1886, ๔. 42. 
Germbejt jtumenerregento finto bie Ertrïge, welofe uns 50m=

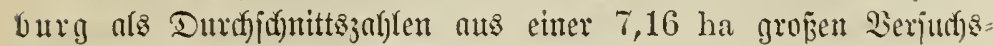

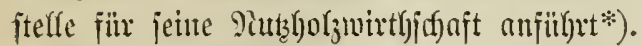

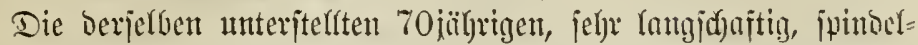

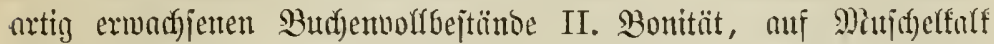

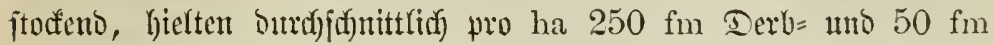

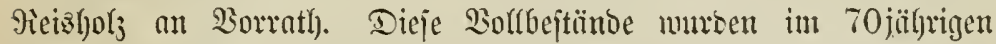

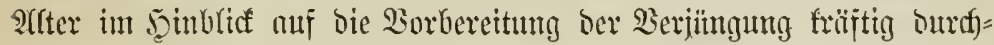
forjtet uno marfen pro ha ab:

In 2)lter won:

55,1 fm Derb $=$ amo 12,6 fm 9ieizlyoly.

73 Jabren folyte Samen $=$ bej.

Srfirmifulagitellumg แno ergab

78 Saljun folgte liute Samen=

joflugitellung mit. $111,2=$ $=12,7=\quad=$

81 Saljren folgte gleidnmiäpige

sidftjulfagjtelfung mit . $84,3=$ $=30,7=$ $83-87$ Jablen Yad)(id)tung unt Freijtellung ber lleber= bülter mit $83,5=\quad=27,0=$ Sd) (iežlidf jtanden nod) pro ha

ca. 35 Heberlyälter mit

$68,5=\quad=26,0=$

Sa. $\underbrace{418,6 \mathrm{fm}}$ Derb $=\overline{\text { Sa. } 113,3 \mathrm{fm}}$ Feişbols

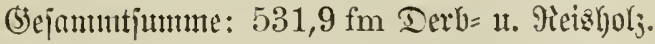

Şalten mir biejen Ertrïgen bie Shtgaben gleidfalter, normtal bejtoderter Buffenbejtünoe II. Bonitüt nad) Banr entgegen, jo finten

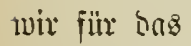

70jüfrige 2(lter ben Borratl) ant Derblyol; ju . . 339,4 fm,

$=$ Derb $=$ mto Tieizljolj ju $415,5=$

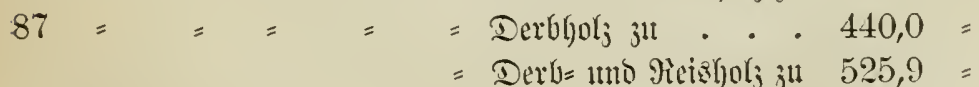

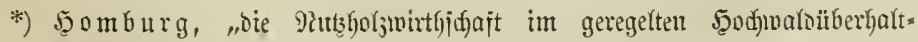

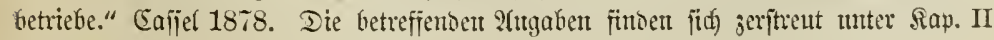
"Die Prariz." 


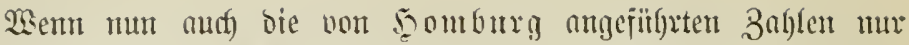

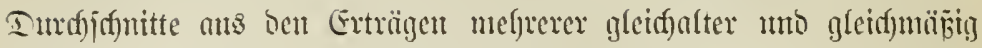

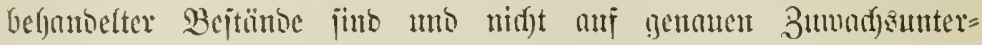
juffuntren berthen, jo mögen jie inumerfint in gropen Bügen bie

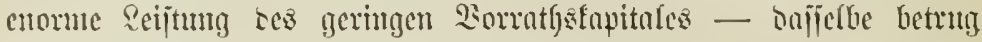

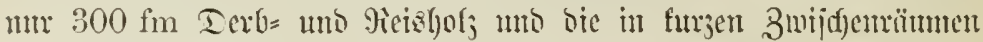

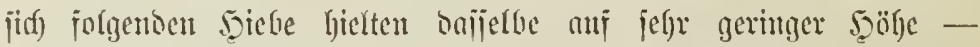

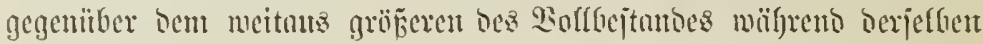

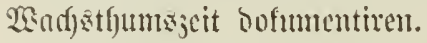

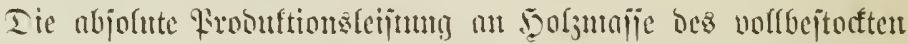
Bejtunes nad) ber Ertragstajel, nümlidf) pro ha $595,9 \mathrm{fm}$ im 2 (tev

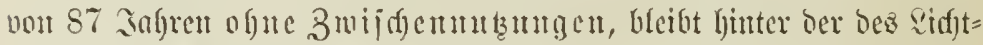

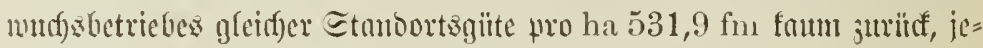

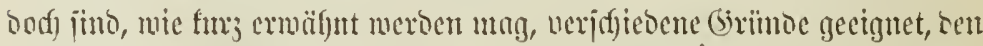

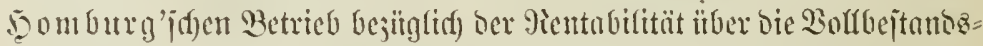

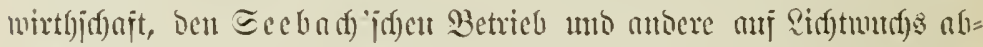

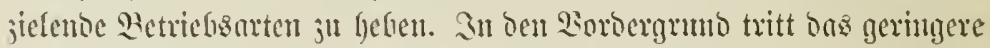

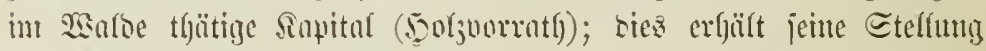

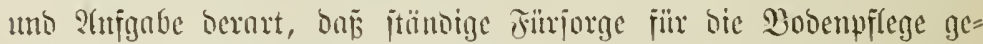

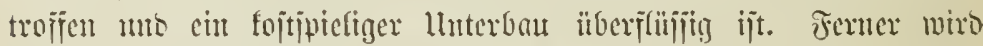

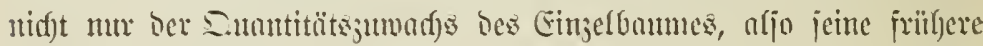

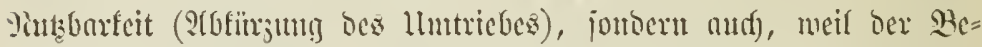

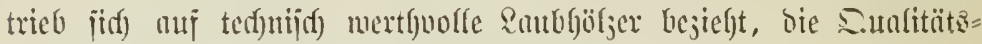

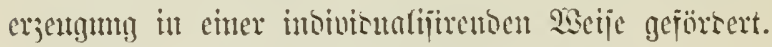

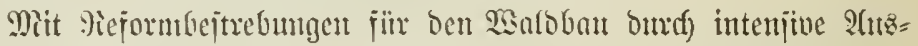

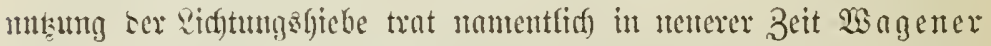

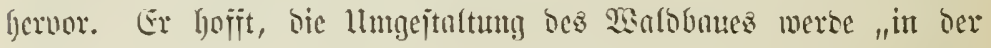

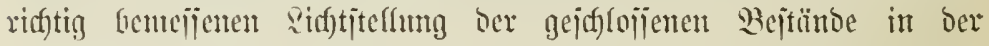

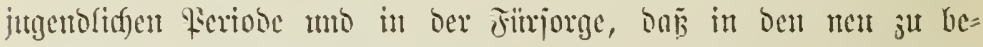
grimbenoen gentijaten Bejtünben bie jpäter sominirentoen @tämme

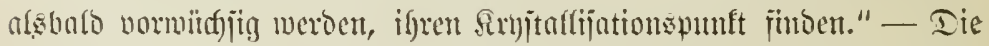

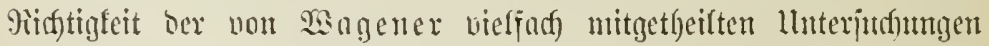

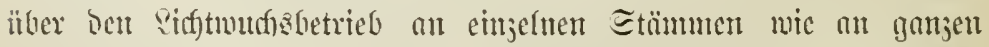

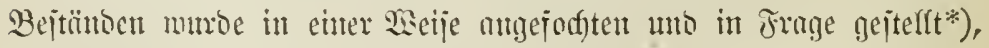

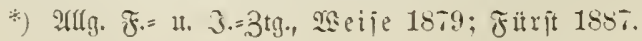




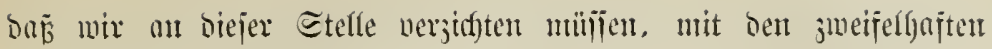
¡ejuftaten ju red)nen.

\section{Mientalilität.}

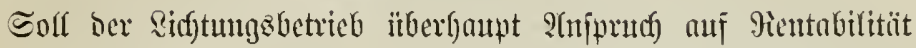
haben, jo milifen wor affent bic Boratsietzungen fïr jeine Beredf $=$ tigung, bas ijt @tandort guter Bonitït*) (I uno II), gegeben jeir

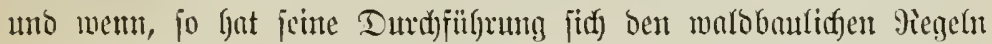

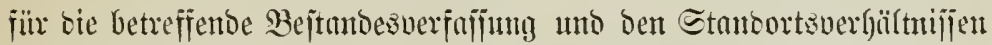

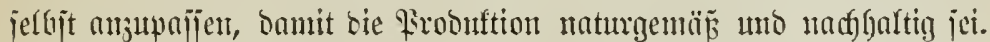

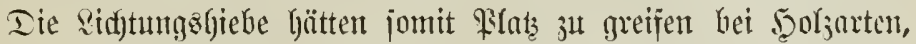

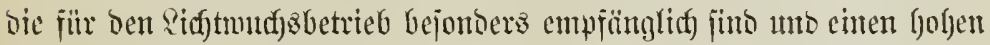

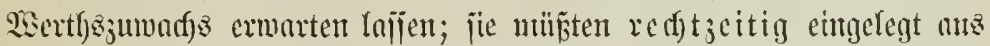

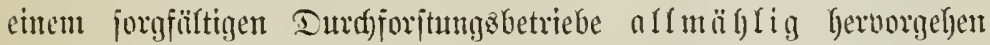

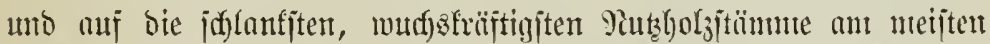

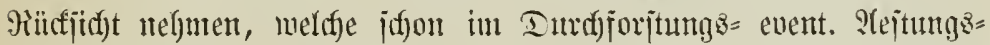

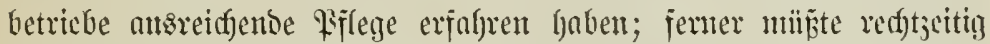

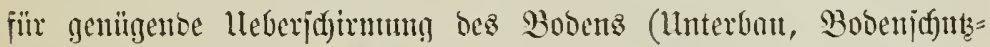

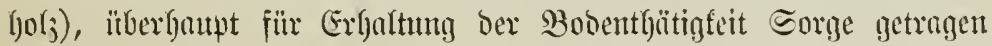
meroen.

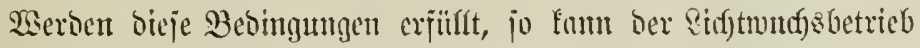
an uno jiin jids jofon - baz ijt, abgejefen vom sidgtungagrabe -

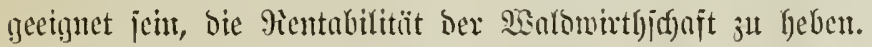

Die Sidjung mur angejelyen weroen als eine Diebsoperation, melde mie bie Durdfforjtung sie Bejtandesglieber bejimirt uno ben

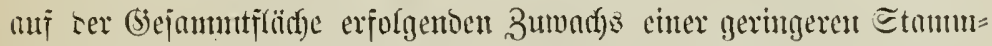

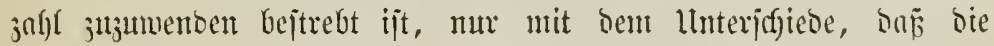

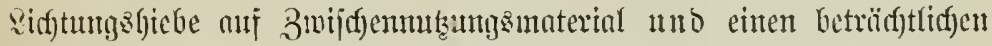

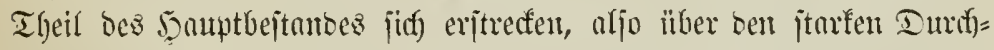

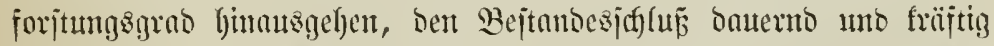

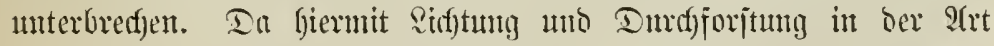

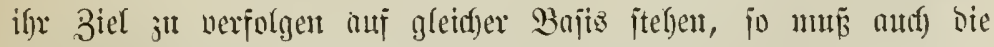

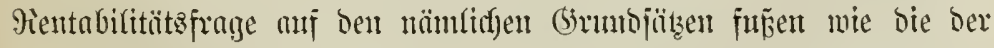

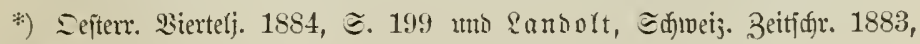
๔. 172 
Iurdjorjtung, mm mitijen eben bie bort nom ?(usfiebe mo jemen

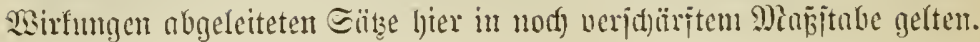

\section{a. 2lusjetyenter Betrieb.}

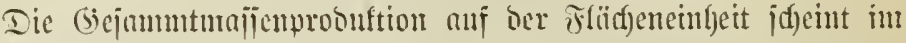

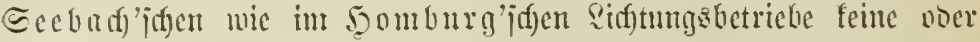
unmejentlich gejteigerte ju jein im 2aergleidfe mit jener bes Bobl=

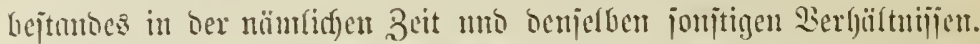
Der böfjere Enomertf) ber Pirobuftion fonmt, ment mun cinen cin= jehten Bajtanto betrad)tet uno bie Daterialmiülle mit ber Beit bes

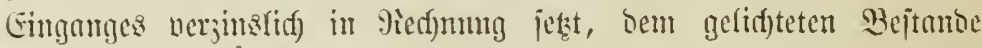
unjueifelfajt ju. Es jtelgen fidf) bei ber Sergleidfung ber beiben

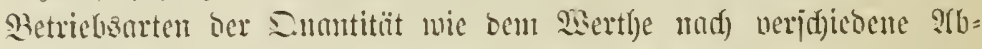

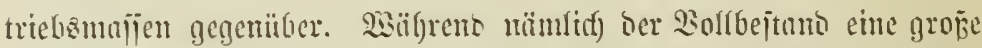

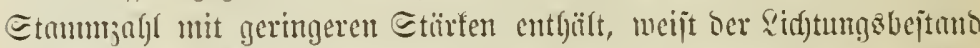

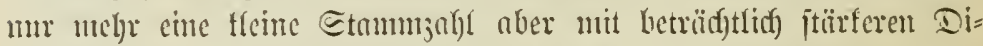

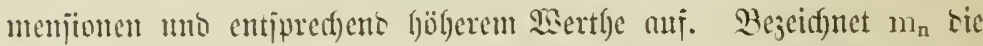

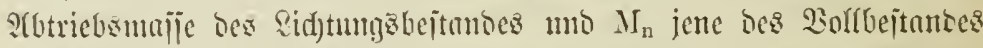

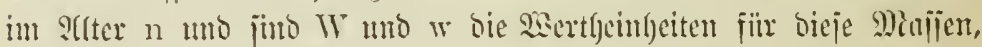

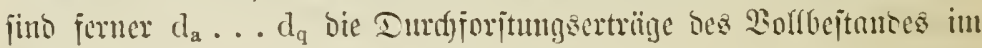

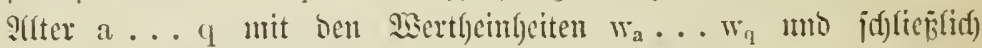

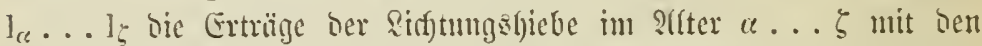

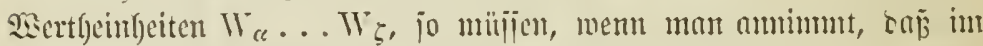

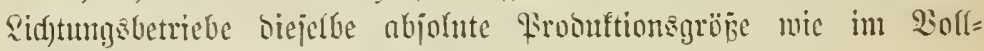
bejtande erfangt wirt, ber Endertrang bes Doflkejtanbes

$$
\mathrm{M}_{\mathrm{n}} \mathrm{w}+\mathrm{d}_{\mathrm{a}} \mathrm{w}_{2} 1,0 \mathrm{p}^{\mathrm{n}-\mathrm{a}}+\ldots \mathrm{d}_{\mathrm{i}} \mathrm{w}_{\mathrm{q}} 1,0 \mathrm{p}^{\mathrm{n}-\mathrm{q}}
$$

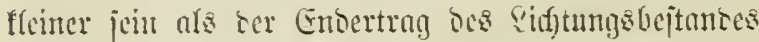

$$
\left.\left.m_{n} W^{*}+1_{c} W_{c} 1,0 p^{n-c}+\ldots\right]_{\zeta} W_{\zeta}+1,0 p^{n-\zeta *}\right)
$$

E(f) on barmá, ถaj bei

$$
\mathrm{N}_{\mathrm{n}}+\mathrm{d}_{\mathrm{a}}+\ldots \mathrm{d}_{\mathrm{q}}=\mathrm{m}_{\mathrm{n}}+\mathrm{l}_{\mathrm{c}}+\ldots \mathrm{I}_{\xi}
$$

bie Errtrïge

$$
\mathrm{I}_{c}+\ldots \mathrm{d}_{\zeta}>\mathrm{d}_{\mathrm{a}}+\ldots \mathrm{d}_{\mathrm{q}}
$$

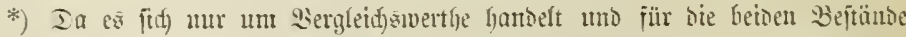

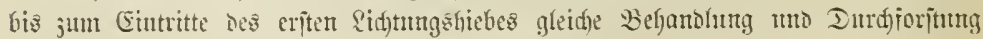

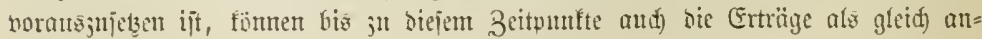
genommen werden uns aū̄er 2trjąz bleiben. 
(geutïs Der Defintion Les Sidtungshiebes) jino, aber ben gleidfen Beitraum ber Derjinjung nom Beitpuntte bes Einganges ab fitr jidf luben, jolgt - aljo ganj abgejeljen nom saerthe - bañ bie ljöhere

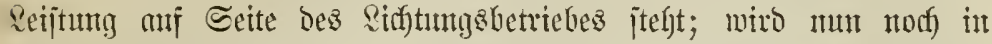

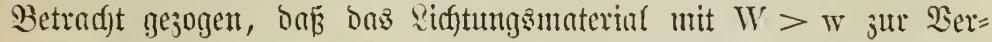

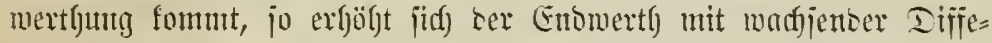

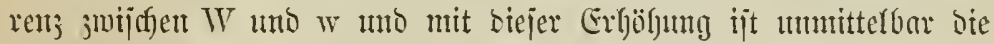

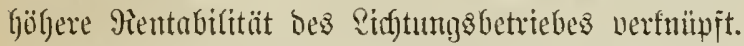

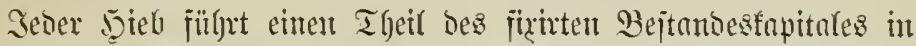

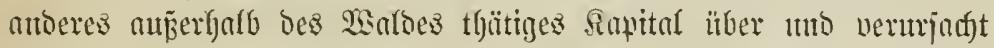
Darurd) nod), ba ein böljeres Firnjent einbringt als ber gejalojiene Bejtant.

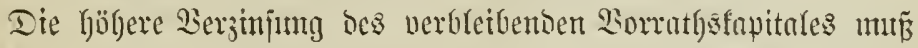
audf bier, wie bei ben Durfforjtungen, fitr jede Sidfuntgsperiobe ab=

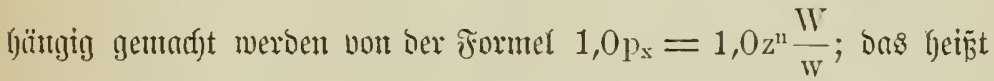

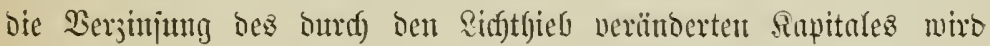

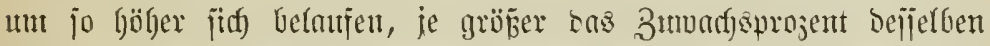

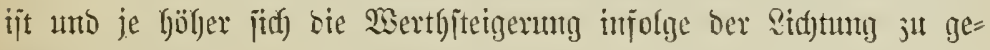
italten vermag.

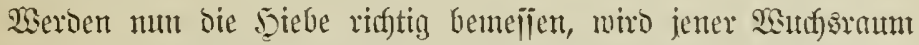

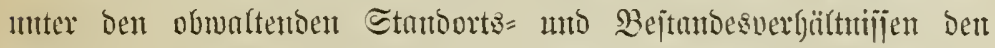

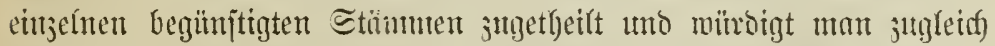

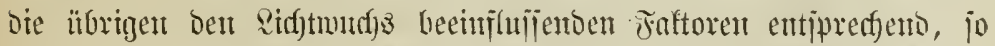

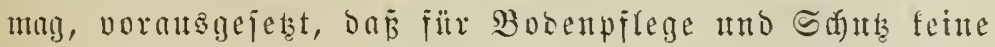

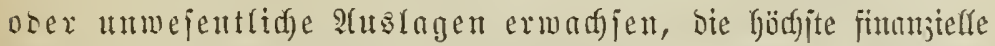

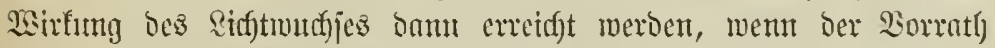

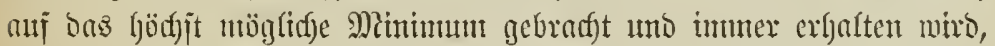

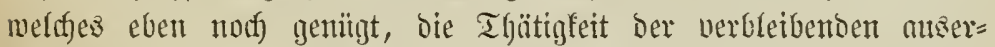

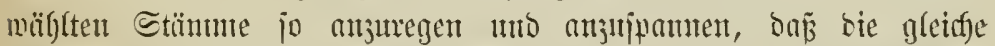
abjohte Bumaffamenge erjetngt wiro wie cet. par. in Bollbejtance.

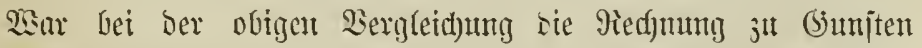

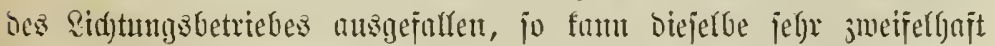

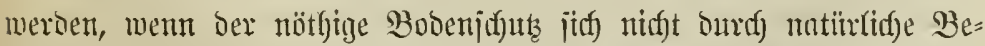

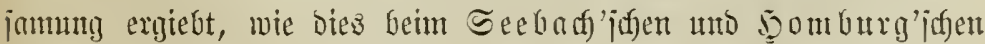
Betriebe ber Fall ijt, jonbern ment zun fïnjtrifjen llnterban, jut 


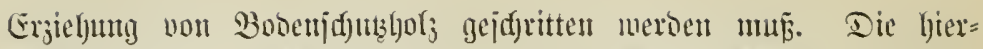

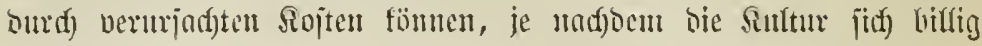

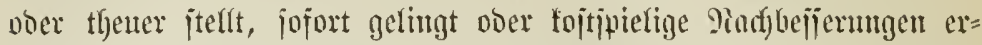

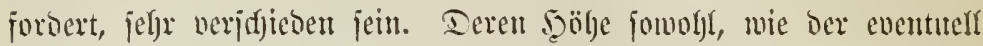
geringe mintljiffaftidfe $23 e r t$ )

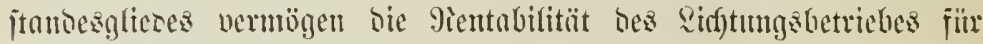

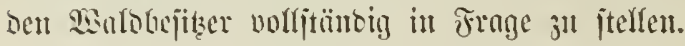

\section{b. 2Tathaltbetricb.}

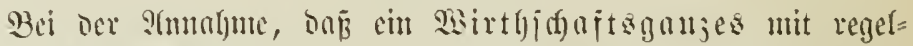

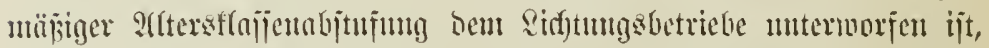

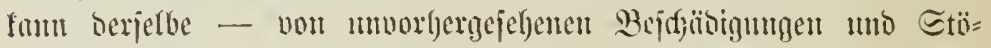

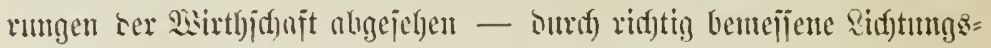

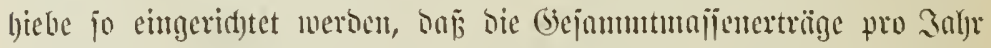

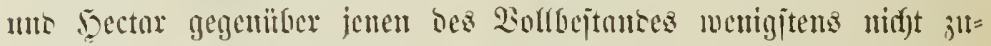

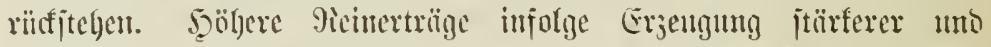
mertfuollerer Dimenjionen in gleidfer Beit ooer bie Ergtebung ber=

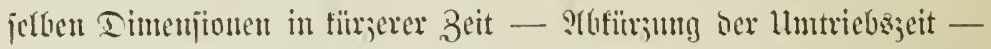

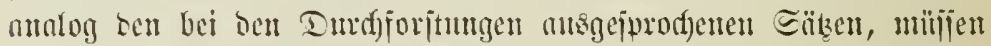

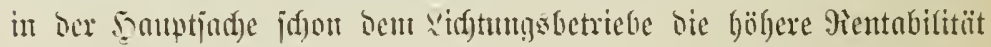

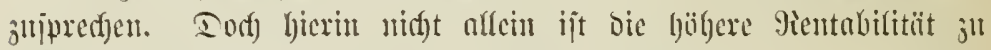

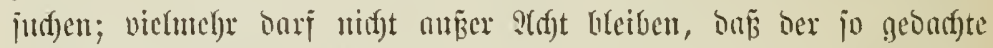
Siffungsuato mit erfebliff geringerem borratbe arbeitet, als ber Bollbejunos=9ormalmalo. Sober cinjelne Bejtans

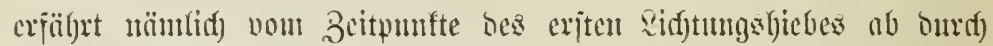

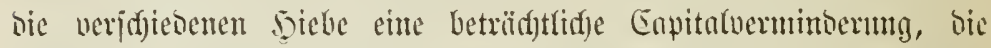

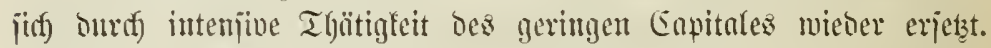

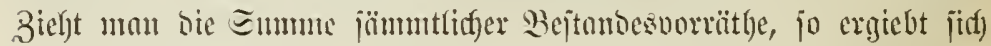

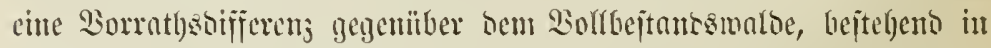

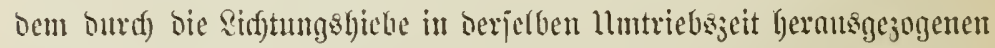
Miaterialnuntum. Llm riejen Betrang ijt bas in Sidntungsbetrielue

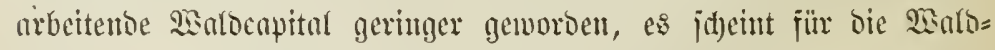

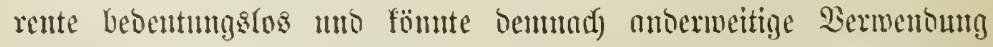

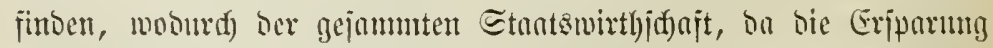
cine jtänrige märe, mu Sortlyeile erwadjen wïrben. Sit biejes

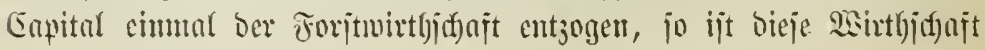


jerbjt barum rentabler gemorden, meil fie, mit einem geringeren

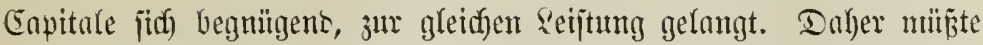
aurf) Dem Rifutungsbetriebe wiebermu nad) Dem nationalöfonomijifen

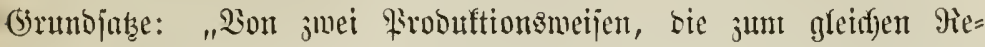
jultate fïflyen, ift iene bie rentablere, weldfe das fleinere Betriegs: capital aufwendet," unhesingt bie lö̈lere Pientubilität juerfaunt meroen.

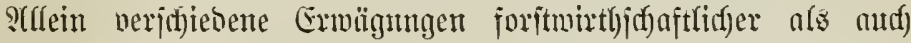

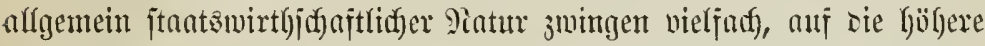

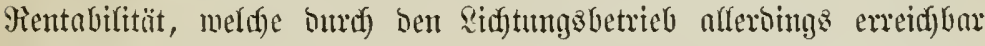

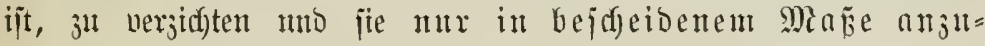
jtreben.

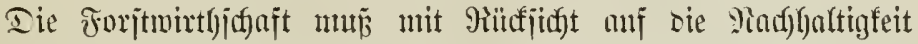
immer eine conjervative jein, jo ba

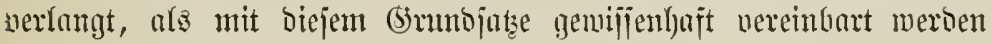

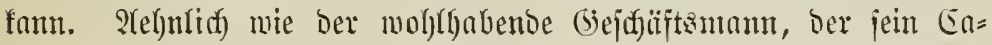
pital in ben nerjofiebenjten \$isertjpapieren angelegt gat uno mit

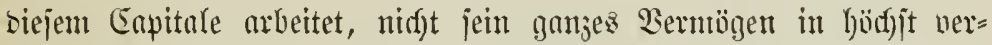
junsficfen Fapieren, bie nur burum fo holjen Bins tragen, meil bas

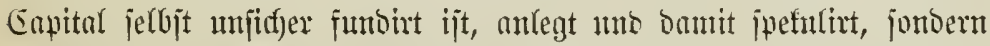

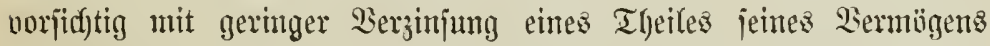

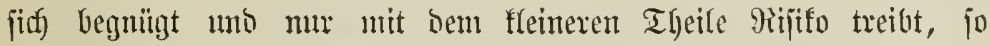

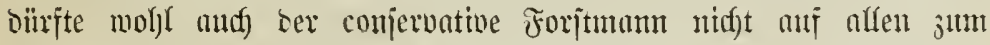

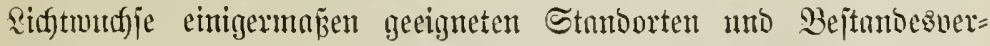
bültnififen ipefuliren; benn es brogt bem sidjtungabletrebe unver=

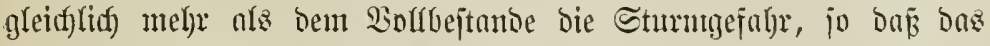
an uno fitr jidf iffon geringe Bejtanbescapital reidft weiter ner=

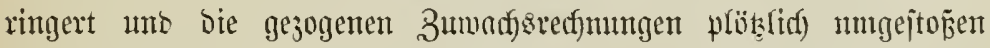
meroen fömen - in einem Hmange, son bie nadyfartige itetige

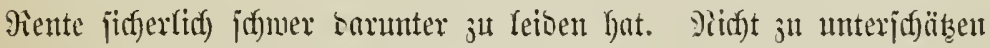

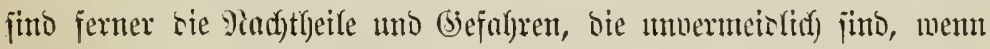

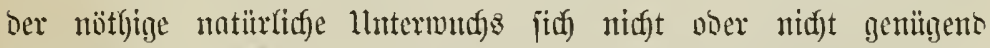

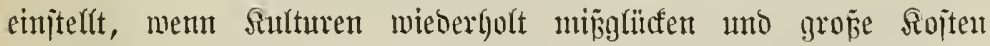

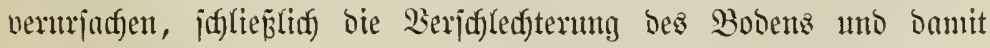

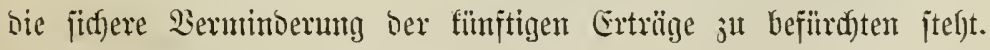
Daljer mäge ber Sidtmudg betrieb nux in Bejtänoen hejter

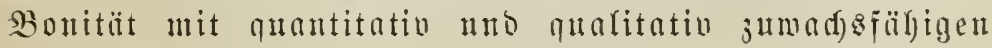




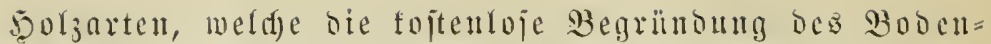
iffulgolzes, bes llnterwudjes ober wenigitens bie jefre

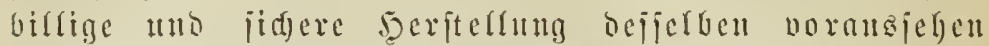

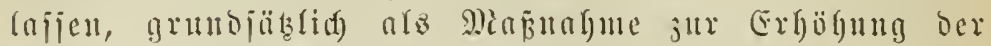

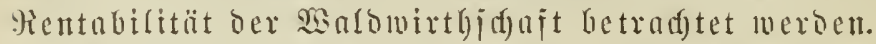

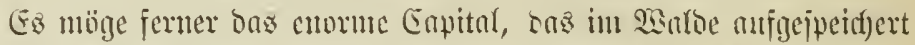

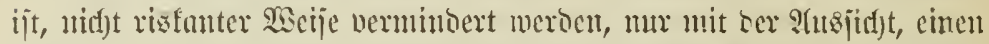

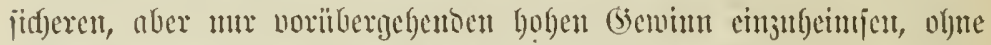

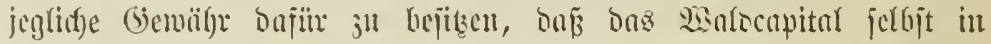

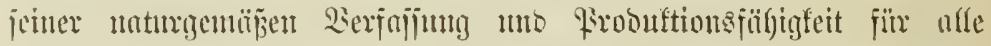

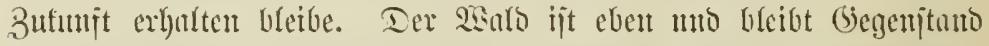

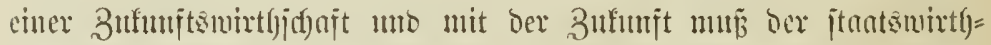

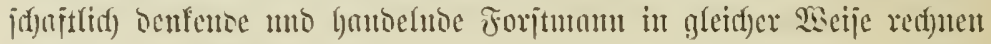

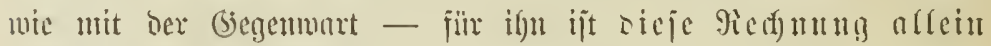
bie forptidf reutable.

2Scun mir im Borfergefenoen cinen geritgen Bettray jux

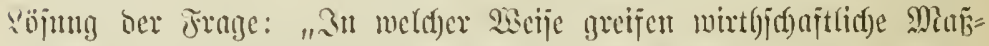

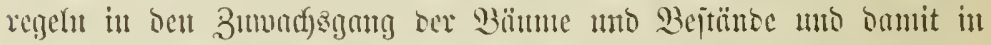

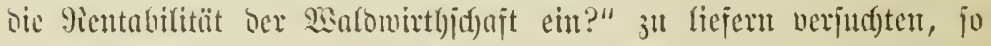

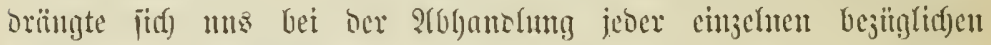

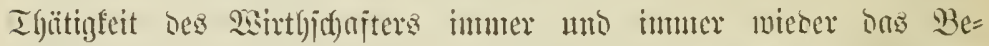

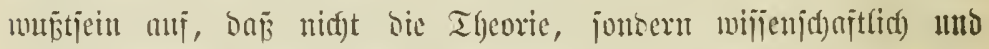

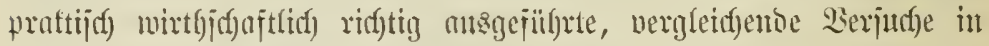

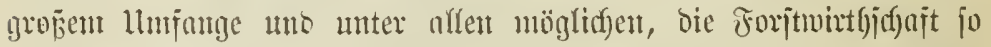

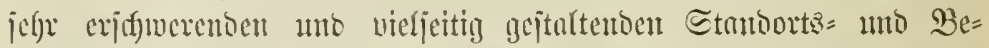

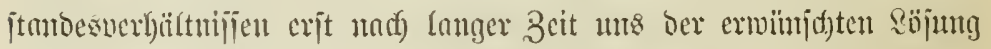
ntïger bringen fömen; ob bie nortiegente Frage bei ber furjen

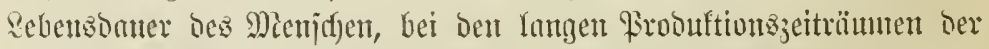

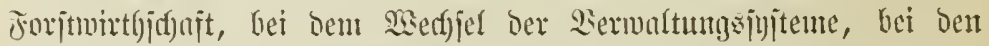

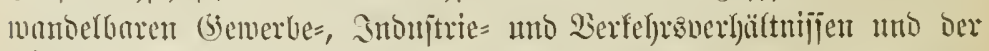

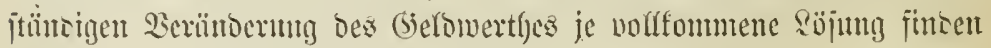
fแนแ - mir mödften $\mathfrak{c}$ bejueifeln. 




\section{LIBRARY \\ UNIVERSITY OF TORONTO}

SD

393

B4
Behringer, Martin

Ueber den Einfluss

wirtschaftlicher Massregeln

BioMed

UNIVERSITY OF TOAONTO LIERARY

$$
[101260]
$$




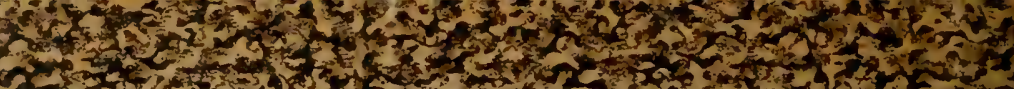

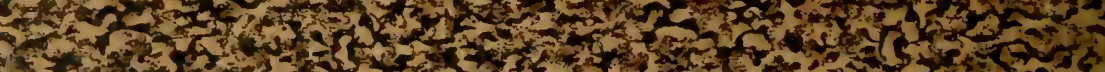

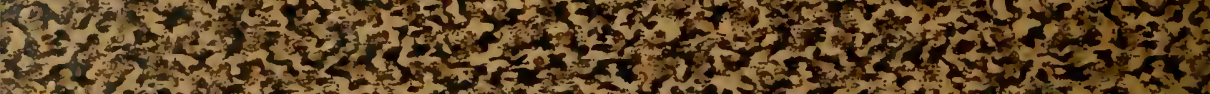

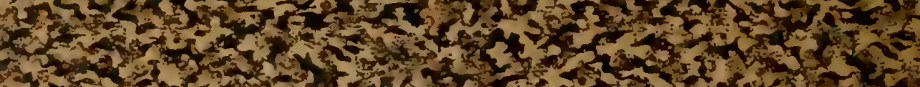

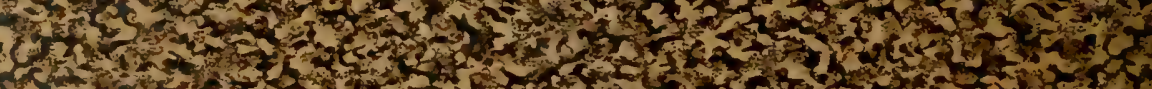

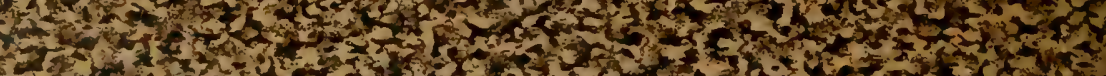

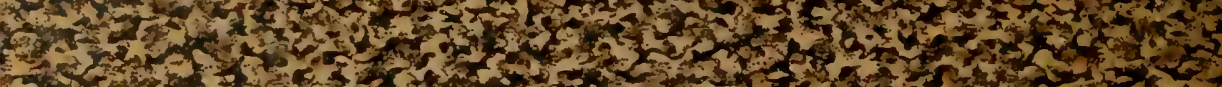

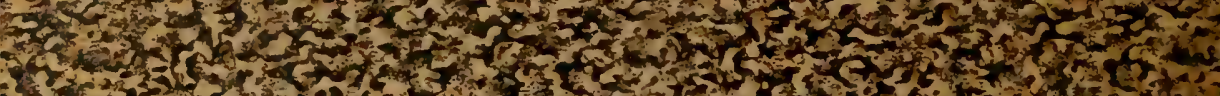

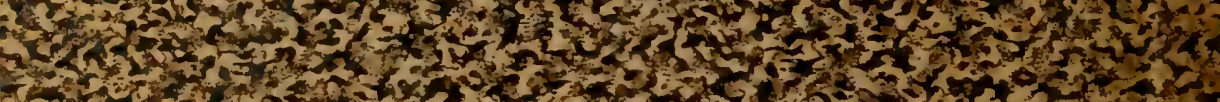

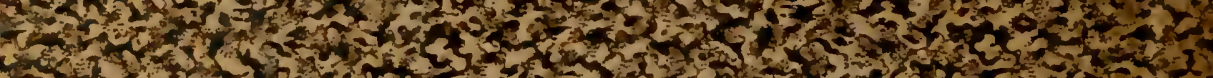

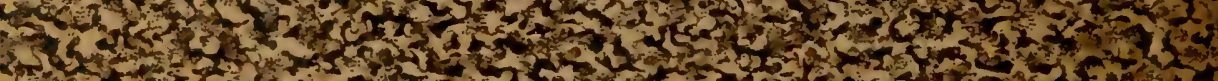

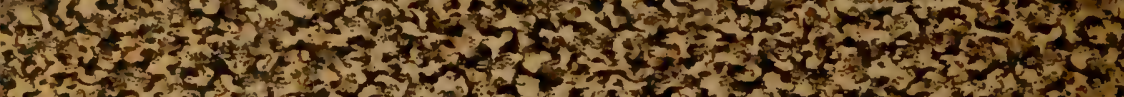

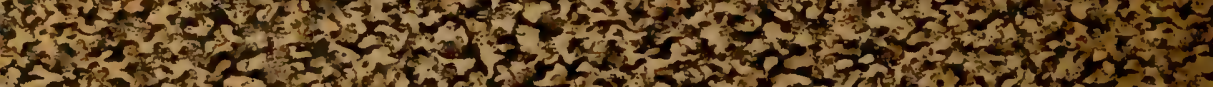

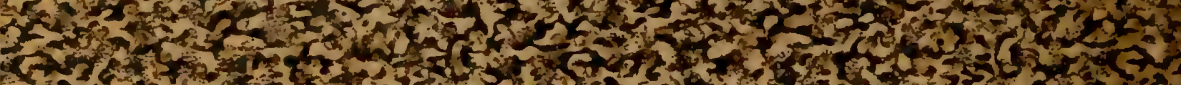

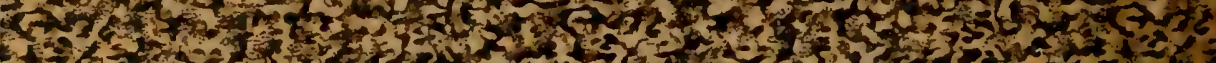

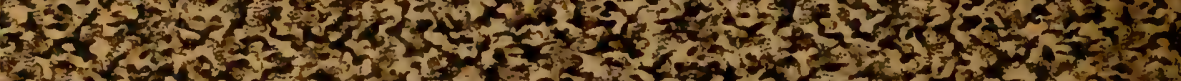

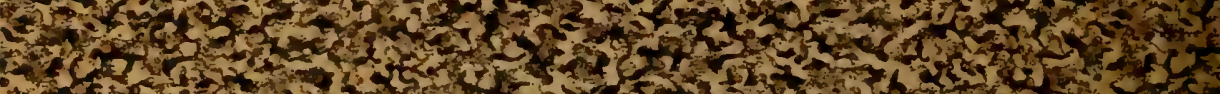

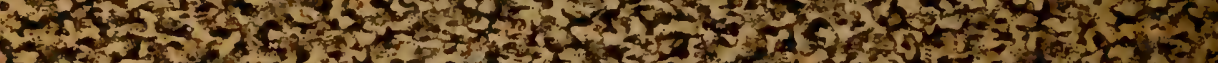

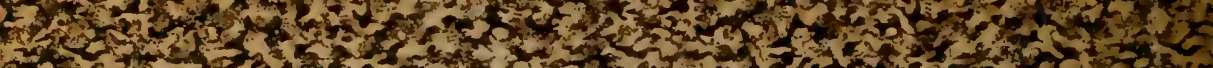

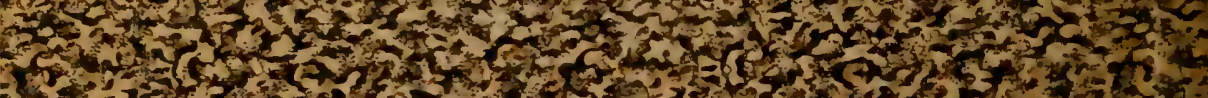

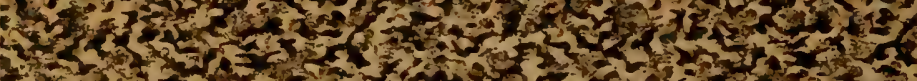

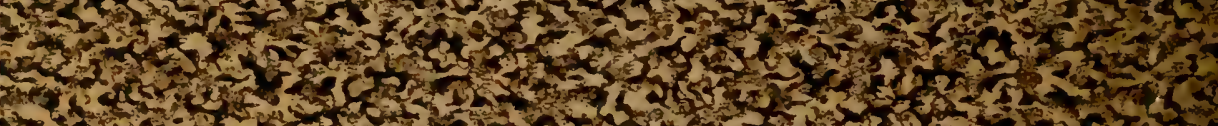

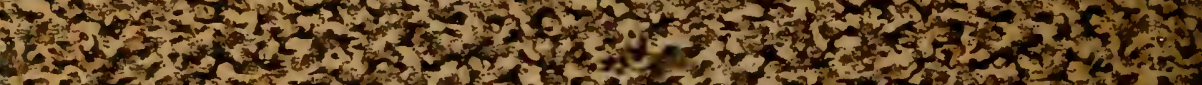

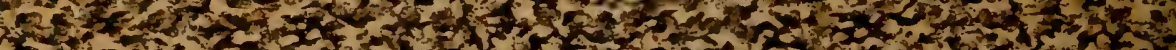

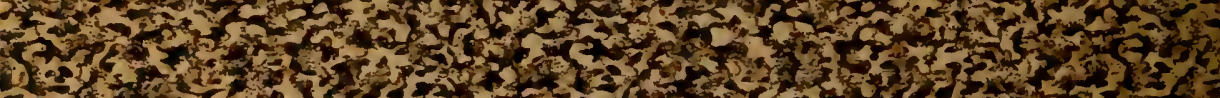

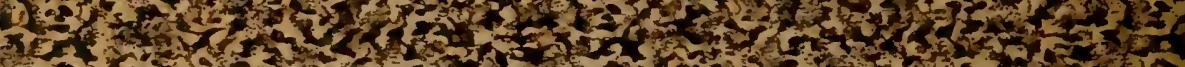

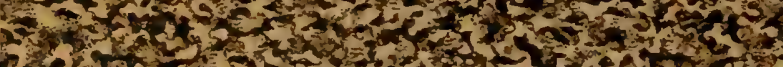

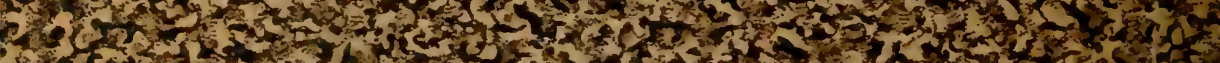

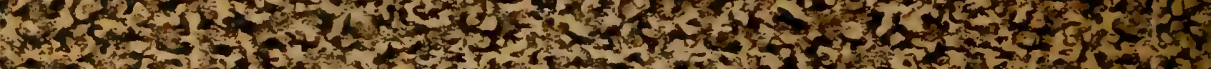

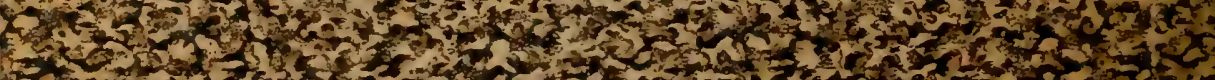

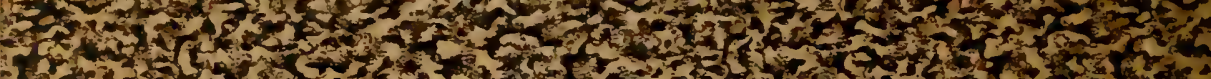

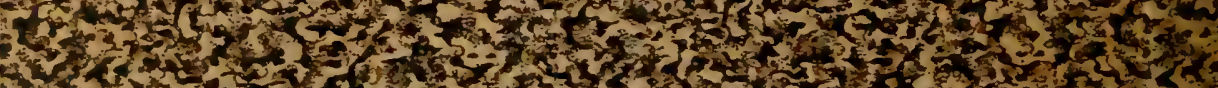

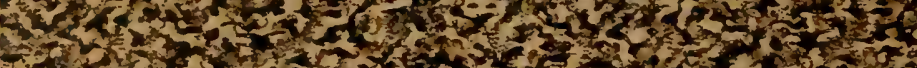

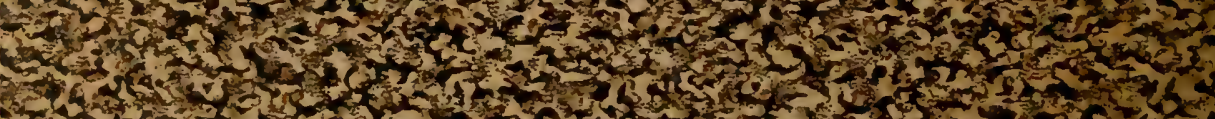

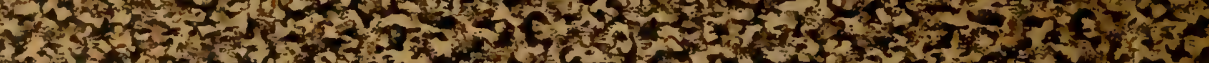

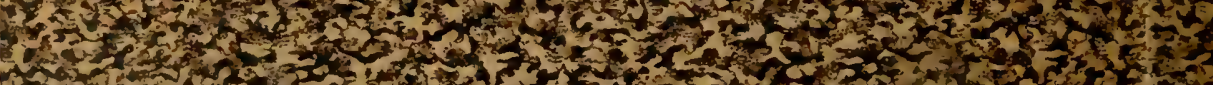

